\title{
Well-posedness for the coupling between a viscous incompressible fluid and an elastic structure
}

\author{
Muriel Boulakia ${ }^{1}$, Sergio Guerrero ${ }^{2}$, and Takéo Takahashi ${ }^{3}$ \\ ${ }^{1}$ Sorbonne Université, Université Paris-Diderot, CNRS, Inria, LJLL, équipe REO, F-75005 \\ Paris \\ ${ }^{2}$ Sorbonne Université, Université Paris-Diderot, CNRS, LJLL, F-75005 Paris \\ ${ }^{3}$ Université de Lorraine, CNRS, Inria, IECL, F-54000 Nancy
}

June 21, 2018

\begin{abstract}
In this paper, we consider a system modeling the interaction between a viscous incompressible fluid and an elastic structure. The fluid motion is represented by the classical Navier-Stokes equations while the elastic displacement is described by the linearized elasticity equation. The elastic structure is immersed in the fluid and the whole system is confined into a general bounded smooth domain of $\mathbb{R}^{3}$. Our main result is the local in time existence and uniqueness of a strong solution of the corresponding system.
\end{abstract}

Keywords: fluid-structure, Navier-Stokes system, elastic structure

2010 Mathematics Subject Classification. 76D03, 76D05, 35Q74, 76D27

\section{Contents}

\begin{tabular}{|lrr}
\hline 1 & Introduction & 2
\end{tabular}

\begin{tabular}{|lll}
2 & Study of the linear system for smooth data & 6
\end{tabular}

$2.1 \quad$ Estimates on the terms coming from $X \mid \ldots \ldots \ldots \ldots \ldots \ldots$

2.2 Estimates on the terms coming from $(\widetilde{v}, \widetilde{q}) \ldots \ldots \ldots \ldots \ldots$. . . . . . . . . . . . . . 9

$\begin{array}{llllll}2.3 & \text { Estimates on } F_{1}, F_{2}, F_{3} & \ldots & \ldots & \ldots & \ldots\end{array} \ldots \ldots \ldots \ldots \ldots \ldots \ldots$

2.4 Proof of Proposition $2.1 \ldots \ldots \ldots \ldots \ldots \ldots \ldots \ldots$

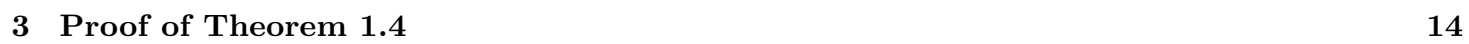

3.1 Energy estimates . . . . . . . . . . . . . . . . . . . . . . . . . . . . 14

3.2 Estimates on the time derivative . . . . . . . . . . . . . . . . . . . . . . . . 15

$3.3 \quad$ Elliptic estimates . . . . . . . . . . . . . . . . . . . . . . . . . . . . . . . . . . . . . . . . . 17

3.4 Study of a linear Stokes system . . . . . . . . . . . . . . . . . . . . . . . . . . . . . . . . 18

3.5 Estimate of the $H^{5 / 2+1 / 8}\left(\Omega_{F}\right)$-norm $\ldots \ldots \ldots \ldots \ldots \ldots \ldots \ldots \ldots \ldots$

3.6 A density argument $\ldots \ldots \ldots \ldots \ldots \ldots \ldots \ldots \ldots \ldots$

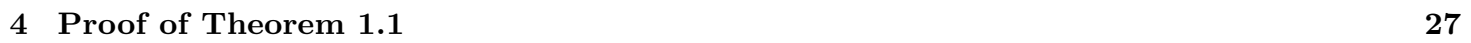

\begin{tabular}{|l|l|}
\hline A Some technical results on Sobolev-Slobodeckij spaces & 28 \\
\hline
\end{tabular}

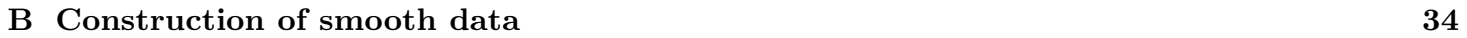




\section{Introduction}

We consider the evolution of a coupled system composed of an incompressible viscous fluid and an elastic structure. The system evolves in a bounded domain that we denote by $\Omega \subset \mathbb{R}^{3}$. At initial time, the structure occupies the domain $\Omega_{S}$ and the fluid occupies the domain $\Omega_{F}=\Omega \backslash \overline{\Omega_{S}}$.

We denote by $\xi$ the elastic displacement of the structure defined on $(0, T) \times \Omega_{S}$. For each time $t$, the domain occupied by the structure is given by $\Omega_{S}(t)=(\mathrm{id}+\xi(t, \cdot))\left(\Omega_{S}\right)$ and the fluid domain is given by $\Omega_{F}(t)=\Omega \backslash \overline{\Omega_{S}(t)}$. We denote by $u$ and $p$ the Eulerian velocity and the pressure of the fluid. At each time $t \in(0, T)$, these functions are defined in $\Omega_{F}(t)$. To come back to the initial configuration, we introduce the characteristic $X$ of the fluid velocity defined on $(0, T) \times \Omega_{F}$ by:

$$
\left\{\begin{aligned}
\partial_{t} X(t, y) & =u(t, X(t, y)) \quad t \in(0, T), \\
X(0, y) & =y
\end{aligned}\right.
$$

We consider that the fluid motion is governed by the incompressible Navier-Stokes equations and that the elastic displacement satisfies the linear elasticity equation. The coupling at the interface is expressed through two conditions: continuity of the velocities and continuity of the normal component of the stress tensors. By this way, we have the following system:

$$
\begin{cases}\partial_{t} u+(u \cdot \nabla) u-\operatorname{div} \mathbb{T}(u, p)=\widetilde{g} & t \in(0, T), x \in \Omega_{F}(t), \\ \operatorname{div} u=0 & t \in(0, T), x \in \Omega_{F}(t), \\ \partial_{t t} \xi-\operatorname{div} \Sigma(\xi)=0 & t \in(0, T), y \in \Omega_{S}, \\ u=0 & t \in(0, T), x \in \partial \Omega, \\ u \circ X=\partial_{t} \xi & t \in(0, T), y \in \partial \Omega_{S}, \\ (\mathbb{T}(u, p) \circ X) \operatorname{Cof}(\nabla X) n=\Sigma(\xi) n & t \in(0, T), y \in \partial \Omega_{S}, \\ u(0, \cdot)=u_{0} & y \in \Omega_{F}, \\ \xi(0, \cdot)=0, \partial_{t} \xi(0, \cdot)=\xi_{1} & y \in \Omega_{S} .\end{cases}
$$

We have denoted by $n$ the outward normal of $\partial \Omega_{S}$, by $\mathbb{T}$ the fluid stress tensor and by $\Sigma$ the elastic stress tensor defined by

$$
\mathbb{T}(u, p)=2 \varepsilon(u)-p I_{3}, \quad \Sigma(\xi)=2 \lambda_{1} \varepsilon(\xi)+\lambda_{2}(\operatorname{div} \xi) I_{3} \text { with } \varepsilon(u)=\frac{1}{2}\left(\nabla u+(\nabla u)^{*}\right) .
$$

We have denoted by $A^{*}$ and by $\operatorname{Cof}(A)$ the transpose and the cofactor matrix of any matrix $A$. In the expression of the fluid stress tensor $\mathbb{T}$, without any loss of generality, we have taken the viscosity of the fluid constant and equal to 1 . The expression of $\Sigma$ involves the Lamé constants $\lambda_{1}$ and $\lambda_{2}$ which are assumed to satisfy:

$$
\lambda_{1}>0, \quad \lambda_{2} \geqslant 0
$$

The function $\widetilde{g}$ is a force applied in the fluid. To simplify, we have not put any force in the elasticity equation and we have also assumed that at initial time we are in the reference configuration, so that the elastic displacement is equal to zero at initial time.

In the system (1.2), the fluid equations are written in the Eulerian framework. By this way, the fluid equations are given in an unknown non cylindrical domain. In a classical way, we use $X$ as a change of variables for the fluid variables to come back to the initial domain $\Omega_{F}$ and we rewrite the fluid equations in the domain $(0, T) \times \Omega_{F}$. More precisely, we define the Lagrangian velocity $v$ and the pressure $q$ over the initial fluid domain by: for all $(t, y) \in(0, T) \times \Omega_{F}$

$$
v(t, y)=u(t, X(t, y)) \quad \text { and } \quad q(t, y)=p(t, X(t, y))
$$

Due to the incompressibility condition satisfied by $u, X$ satisfies the volume-preserving property 
$\operatorname{det}(\nabla X)=1$. Then, using this property, we can rewrite 1.2 together with (1.1) as

$$
\begin{cases}\partial_{t} v-\operatorname{div} \mathbb{T}_{X}(v, q)=g & \text { in }(0, T) \times \Omega_{F}, \\ \nabla v: \operatorname{Cof}(\nabla X)=0 & \text { in }(0, T) \times \Omega_{F}, \\ \partial_{t t} \xi-\operatorname{div} \Sigma(\xi)=0 & \text { in }(0, T) \times \Omega_{S}, \\ \partial_{t} X=v & \text { in }(0, T) \times \Omega_{F}, \\ v=\partial_{t} \xi & \text { on }(0, T) \times \partial \Omega_{S}, \\ v=0 & \text { on }(0, T) \times \partial \Omega, \\ \mathbb{T}_{X}(v, q) n=\Sigma(\xi) n & \text { on }(0, T) \times \partial \Omega_{S}, \\ v(0, \cdot)=v_{0}, \quad X(0, \cdot)=\text { id } & \text { in } \Omega_{F}, \\ \xi(0, \cdot)=0, \partial_{t} \xi(0, \cdot)=\xi_{1} & \text { in } \Omega_{S},\end{cases}
$$

where for $(t, y) \in(0, T) \times \Omega_{F}$,

$$
v_{0}(y)=u_{0}(y), \quad g(t, y)=\widetilde{g}(t, X(t, y))
$$

and

$$
\mathbb{T}_{X}(v, q):=\left[(\nabla v) \operatorname{Cof}(\nabla X)^{*}+\operatorname{Cof}(\nabla X)(\nabla v)^{*}-q I_{3}\right] \operatorname{Cof}(\nabla X)
$$

In the above system and in what follows, we use the notation $A: B:=\operatorname{tr}\left(A B^{*}\right)$ for $3 \times 3$ matrices $A$ and $B$.

We assume that $\Omega_{F}$ is a $C^{2}$ domain and that the data $v_{0}, \xi_{1}$ and $g$ satisfy the following conditions:

$$
\begin{gathered}
v_{0} \in H^{2}\left(\Omega_{F}\right), \quad \xi_{1} \in H^{1+1 / 8}\left(\Omega_{S}\right), \quad g \in H^{1}\left(0, T ; L^{2}\left(\Omega_{F}\right)\right) \cap L^{2}\left(0, T ; H^{1 / 2+1 / 8}\left(\Omega_{F}\right)\right), \\
\operatorname{div} g=0 \quad \text { in }(0, T) \times \Omega_{F}, \quad g \cdot n=0 \quad \text { on }(0, T) \times \partial \Omega .
\end{gathered}
$$

Furthermore, we assume the following compatibility conditions: there exist $v_{1} \in L^{2}\left(\Omega_{F}\right)$ and $q_{0} \in$ $H^{1}\left(\Omega_{F}\right)$ such that

$$
\begin{cases}v_{1}-\operatorname{div} \mathbb{T}\left(v_{0}, q_{0}\right)=g(0, \cdot) & \text { in } \Omega_{F}, \\ \operatorname{div} v_{0}=0 & \text { in } \Omega_{F}, \\ \operatorname{div} v_{1}=\left(\nabla v_{0}\right):\left(\nabla v_{0}\right)^{*} & \text { in } \Omega_{F}, \\ v_{0}=\xi_{1} & \text { on } \partial \Omega_{S}, \\ v_{0}=0 & \text { on } \partial \Omega_{,} \\ v_{1} \cdot n=0 & \text { on } \partial \Omega_{F}, \\ \mathbb{T}\left(v_{0}, q_{0}\right) n=0 & \text { on } \partial \Omega_{S} .\end{cases}
$$

Note that the incompressibility and boundary conditions satisfied by $v_{0}$ and $v_{1}$ imply in particular

$$
\int_{\partial \Omega_{S}} \xi_{1} \cdot n d \gamma=0, \quad \int_{\partial \Omega_{S}}\left[\left(v_{0} \cdot \nabla\right) v_{0}\right] \cdot n d \gamma=0
$$

Before stating our main result, we introduce the following spaces for our solutions:

$$
\begin{gathered}
\mathcal{S}_{1, T}:=C^{2}\left(L^{2}\left(\Omega_{F}\right)\right) \cap H^{2}\left(H^{1}\left(\Omega_{F}\right)\right) \cap C^{1}\left(H^{2}\left(\Omega_{F}\right)\right) \cap H^{1}\left(H^{5 / 2+1 / 8}\left(\Omega_{F}\right)\right), \\
\mathcal{S}_{2, T}:=C^{1}\left(L^{2}\left(\Omega_{F}\right)\right) \cap H^{1+1 / 3}\left(L^{2}\left(\Omega_{F}\right)\right) \cap H^{1}\left(H^{1}\left(\Omega_{F}\right)\right) \cap C^{0}\left(H^{2}\left(\Omega_{F}\right)\right) \cap L^{2}\left(H^{5 / 2+1 / 8}\left(\Omega_{F}\right)\right), \\
\mathcal{S}_{3, T}:=C^{0}\left(H^{1}\left(\Omega_{F}\right)\right) \cap H^{1 / 3}\left(H^{1}\left(\Omega_{F}\right)\right) \cap L^{2}\left(H^{3 / 2+1 / 8}\left(\Omega_{F}\right)\right), \\
\mathcal{S}_{4, T}:=C^{2}\left(L^{2}\left(\Omega_{S}\right)\right) \cap C^{1}\left(H^{1+1 / 8}\left(\Omega_{S}\right)\right) \cap C^{0}\left(H^{2+1 / 8}\left(\Omega_{S}\right)\right) .
\end{gathered}
$$

To shorten the notation of the functional spaces, here and in what follows, we omit the time interval $(0, T)$ for the Sobolev and Lebesgue spaces and $[0, T]$ for the spaces of $C^{k}$ functions. In all these spaces, we consider the canonical norms.

The main result of our paper states the local in time existence and uniqueness of a regular solution:

Theorem 1.1. Assume that (1.7)-(1.9) holds. Then, there exists a time

$$
T=T\left(\left\|v_{0}\right\|_{H^{2}\left(\Omega_{F}\right)},\left\|\xi_{1}\right\|_{H^{1+1 / 8}\left(\Omega_{S}\right)},\|g\|_{H^{1}\left(L^{2}\left(\Omega_{F}\right)\right) \cap C^{0}\left(L^{2}\left(\Omega_{F}\right)\right) \cap L^{2}\left(H^{1 / 2+1 / 8}\left(\Omega_{F}\right)\right)}\right)>0
$$

such that system 1.5 possesses a unique solution $(X, v, q, \xi)$ on $(0, T)$ with the following regularity

$$
(X, v, q, \xi) \in \mathcal{S}_{1, T} \times \mathcal{S}_{2, T} \times \mathcal{S}_{3, T} \times \mathcal{S}_{4, T}
$$

Moreover, $X(t, \cdot): \Omega_{F} \rightarrow \Omega_{F}(t)$ is a diffeomorphism for all $t \in(0, T)$. 
Remark 1.2. In Theorem 1.1, we impose that $g$ satisfies condition (1.8). This hypothesis is technical and may be not necessary. It is only used in the construction of regular data approximating $\left(v_{0}, \xi, g\right)(s e e$ Section B). In fact, we only need the condition (1.8) at $t=0$.

Many works have been already devoted to the well-posedness of system [1.5. One of the difficulties in the study of this system is due to the fact that it couples two equations of different nature. Another important difficulty comes from the fact that the fluid domain is variable in time and its evolution is given by the elastic displacement, which is one of the unknowns of the problem.

Due to these difficulties, several studies have analyzed approximated systems of (1.5), for instance by adding a regularizing term in the elasticity equation (see 4]) or by discretizing the elasticity equation (see 11] for weak solutions and [7] for strong solutions).

One of the first works tackling the existence of strong solutions of $[1.5$ is 9 ] (see also [10]). In this article, the authors obtain the existence of local strong solutions for initial conditions having the following regularity

$$
\left(v_{0}, \xi_{1}\right) \in H^{5}\left(\Omega_{F}\right) \times H^{2}\left(\Omega_{S}\right)
$$

and with several compatibility conditions. They also obtain the uniqueness of strong solutions if they increase the regularity of the initial conditions:

$$
\left(v_{0}, \xi_{1}\right) \in H^{7}\left(\Omega_{F}\right) \times H^{4}\left(\Omega_{S}\right)
$$

with again the corresponding compatibility conditions.

In a particular case where the initial interface between the fluid and the solid is flat, [18] and [25] have obtained the local in time existence of strong solutions. In [18, the authors consider initial conditions with the following regularity

$$
\left(v_{0}, \xi_{1}\right) \in H^{3}\left(\Omega_{F}\right) \times H^{2}\left(\Omega_{S}\right) .
$$

In 25], the authors assume that the initial conditions satisfy

$$
\left(v_{0}, \xi_{1}\right) \in H^{3 / 2+\varepsilon}\left(\Omega_{F}\right) \times H^{1+\varepsilon}\left(\Omega_{S}\right)
$$

for $\varepsilon>0$ arbitrary small. They also need to impose periodic boundary conditions in the two first directions.

With respect to the above results, we bring several improvements to the theory of strong solutions for system 1.5. First, we need weaker regularity assumptions on the data than in 9 and in [18. Second, in comparison to 25], we do not need the initial interface between the fluid and the solid to be flat (or the boundary conditions to be periodic in $x_{1}$ and $x_{2}$ ). Finally, let us mention that, as pointed out in [25, the proofs in [9] and in [18] do not seem to be complete.

Let us also quote 20 in the case of a non-moving fluid domain. We can refer to some works that have been done for related systems: for instance, [3, [5, 6], [19, [17] in the case of a viscous compressible fluid and [2], 8], 12], 24], 13, 1], 14] in the case where the elastic structure is replaced by a beam or a plate located on a part of the fluid boundary.

To prove Theorem 1.1. we will first consider a linear system associated with 1.5 and study the wellposedness of this linear system. We will then use a fixed-point argument to come back to the nonlinear problem 1.5. The linear system will be obtained by replacing the flow $X$ by a given flow $\widehat{X}$ in the expression of $\mathbb{T}_{X}$ and in the term $\operatorname{Cof}(\nabla X)$ which appears in $\left.\sqrt{1.5}\right)_{2}$. More precisely, to introduce our linear system, we define, for $M>0$, the closed subset $B_{M}$ of $\mathcal{S}_{1, T}$ by

$$
B_{M}:=\left\{\widehat{X} \in \mathcal{S}_{1, T} ;\|\widehat{X}\|_{\mathcal{S}_{1, T}} \leqslant M, \widehat{X}(0, \cdot)=\mathrm{id}, \partial_{t} \widehat{X}(0, \cdot)=v_{0}\right\} .
$$

We assume that $M$ is large enough so that $B_{M}$ is non empty and in particular we assume in what follows that

For $\widehat{X} \in B_{M}$, we write

$$
\left\|v_{0}\right\|_{H^{2}\left(\Omega_{F}\right)} \leqslant M
$$

$$
\widehat{v}:=\partial_{t} \widehat{X}
$$

so that

$$
\widehat{X}(t, y)=y+\int_{0}^{t} \widehat{v}(s, y) d s .
$$

Remark 1.3. Note that we do not need to impose that $\widehat{X}$ satisfies the condition $\operatorname{det}(\nabla X)=1$ which holds for $X$ solution of the nonlinear problem. 
For a given $\widehat{X} \in B_{M}$, we consider the linearized system associated with 1.5 :

$$
\begin{cases}\partial_{t} v-\operatorname{div} \mathbb{T}_{\widehat{X}}(v, q)=G & \text { in }(0, T) \times \Omega_{F}, \\ \nabla v: \operatorname{Cof}(\nabla \widehat{X})=0 & \text { in }(0, T) \times \Omega_{F}, \\ \partial_{t t} \xi-\operatorname{div} \Sigma(\xi)=0 & \text { in }(0, T) \times \Omega_{S}, \\ \partial_{t} X=v & \text { in }(0, T) \times \Omega_{F}, \\ v=0 & \text { on }(0, T) \times \partial \Omega, \\ v=\partial_{t} \xi & \text { on }(0, T) \times \partial \Omega_{S}, \\ \mathbb{T}_{\widehat{X}}(v, q) n=\Sigma(\xi) n & \text { on }(0, T) \times \partial \Omega_{S}, \\ v(0, \cdot)=V_{0}, \quad X(0, \cdot)=\text { id } & \text { in } \Omega_{F}, \\ \xi(0, \cdot)=0, \partial_{t} \xi(0, \cdot)=\Xi_{1} & \text { in } \Omega_{S} .\end{cases}
$$

We recall that $\mathbb{T}_{\widehat{X}}$ is defined by $(1.6)$. For this linear system, we will prove the existence and uniqueness of solution in the same functional spaces as in our main Theorem 1.1. More precisely, let us consider

$$
\begin{gathered}
V_{0} \in H^{2}\left(\Omega_{F}\right), \quad \Xi_{1} \in H^{1+1 / 8}\left(\Omega_{S}\right), \quad G \in H^{1}\left(L^{2}\left(\Omega_{F}\right)\right) \cap L^{2}\left(H^{1 / 2+1 / 8}\left(\Omega_{F}\right)\right), \\
\operatorname{div} G=0 \quad \text { in }(0, T) \times \Omega_{F}, \quad G \cdot n=0 \quad \text { on }(0, T) \times \partial \Omega,
\end{gathered}
$$

with the following compatibility conditions: there exist $V_{1} \in L^{2}\left(\Omega_{F}\right)$ and $Q_{0} \in H^{1}\left(\Omega_{F}\right)$ such that

$$
\begin{cases}V_{1}-\operatorname{div} \mathbb{T}\left(V_{0}, Q_{0}\right)=G(0, \cdot) & \text { in } \Omega_{F}, \\ \operatorname{div} V_{0}=0 & \text { in } \Omega_{F}, \\ \operatorname{div} V_{1}=\left(\nabla V_{0}\right):\left(\nabla v_{0}\right)^{*} & \text { in } \Omega_{F}, \\ V_{0}=\Xi_{1} & \text { on } \partial \Omega_{S}, \\ V_{0}=0 & \text { on } \partial \Omega, \\ V_{1} \cdot n=0 & \text { on } \partial \Omega_{F}, \\ \mathbb{T}\left(V_{0}, Q_{0}\right) n=0 & \text { on } \partial \Omega_{S} .\end{cases}
$$

As for $\left(v_{0}, \xi_{1}, g\right)$, the above conditions yield

$$
\int_{\partial \Omega_{S}} \Xi_{1} \cdot n d \gamma=0, \quad \int_{\partial \Omega_{S}}\left[\left(v_{0} \cdot \nabla\right) V_{0}\right] \cdot n d \gamma=0 .
$$

The result is stated in the following theorem:

Theorem 1.4. Let us consider $\widehat{X} \in B_{M}$ and $\left(V_{0}, \Xi_{1}, G\right)$ satisfying $\sqrt{1.23}-(1.25)$. Then, there exist $\alpha>0$ and $C>0$ such that for all $T$ satisfying

$$
T^{\alpha}(1+M) \leqslant C
$$

the system 1.14 -1.22 possesses a unique solution $(X, v, q, \xi)$ on $(0, T)$ with the following regularity

$$
(X, v, q, \xi) \in \mathcal{S}_{1, T} \times \mathcal{S}_{2, T} \times \mathcal{S}_{3, T} \times \mathcal{S}_{4, T} .
$$

Moreover, $X(t, \cdot): \Omega_{F} \rightarrow \Omega_{F}(t)$ is a diffeomorphism for all $t \in(0, T)$ and we have the estimate

$$
\begin{aligned}
\|v\|_{\mathcal{S}_{2, T}}+\|q\|_{\mathcal{S}_{3, T}}+\|\xi\|_{\mathcal{S}_{4, T}} \leqslant C\left(\left(1+\left\|v_{0}\right\|_{H^{2}\left(\Omega_{F}\right)}\right)\left\|V_{0}\right\|_{H^{2}\left(\Omega_{F}\right)}+\left\|\Xi_{1}\right\|_{H^{1+1 / 8}\left(\Omega_{S}\right)}\right. \\
\left.+\|G\|_{H^{1}\left(L^{2}\left(\Omega_{F}\right)\right) \cap C^{0}\left(L^{2}\left(\Omega_{F}\right)\right) \cap L^{2}\left(H^{1 / 2+1 / 8}\left(\Omega_{F}\right)\right)}\right) .
\end{aligned}
$$

The proof of Theorem 1.4 constitutes the main part of our paper. To prove this result, we will first assume that the data $V_{0}, \Xi_{1}$ and $G$ are more regular (see hypothesis (2.1)-2.3) and, under these hypotheses, we prove in Section 2 that system 1.14- 1.22 admits a unique smooth solution. This result is stated in Proposition 2.1 and is proved using a fixed point argument. Next, the goal of Section 3 is to weaken the regularity hypotheses on the data to come back to hypothesis $\sqrt{1.23}-(1.25)$. To do so, we prove that the smooth solution obtained in Section 2 satisfies estimate (1.28) which involves in the right-hand side the weaker norms on the data. The proof of this estimate is done in several steps presented from subsection 3.1 to subsection 3.5 . Then, a density argument presented in subsection 3.6 allows us to conclude the proof of Theorem 1.4 


\section{Study of the linear system for smooth data}

In this section, we assume that the data associated to the linear system $[1.14]-1.22$ are smooth data. We assume here

$$
\begin{gathered}
V_{0} \in H^{3}\left(\Omega_{F}\right), \quad \Xi_{1} \in H^{3 / 2+1 / 8}\left(\Omega_{S}\right), \\
G \in H^{1}\left(L^{2}\left(\Omega_{F}\right)\right) \cap L^{2}\left(H^{1 / 2+1 / 8}\left(\Omega_{F}\right)\right), \quad G_{0}:=G(0, \cdot) \in H^{1}\left(\Omega_{F}\right), \\
\operatorname{div} G=0 \quad \text { in }(0, T) \times \Omega_{F},
\end{gathered}
$$

with the following compatibility conditions: there exist $V_{1} \in H^{1}\left(\Omega_{F}\right)$ and $Q_{0} \in H^{2}\left(\Omega_{F}\right)$ such that

$$
\begin{cases}V_{1}-\operatorname{div} \mathbb{T}\left(V_{0}, Q_{0}\right)=G_{0} & \text { in } \Omega_{F}, \\ \operatorname{div} V_{0}=0 & \text { in } \Omega_{F}, \\ \operatorname{div} V_{1}=\left(\nabla V_{0}\right):\left(\nabla v_{0}\right)^{*} & \text { in } \Omega_{F}, \\ V_{0}=\Xi_{1} & \text { on } \partial \Omega_{S}, \\ V_{0}=0 & \text { on } \partial \Omega, \\ V_{1}=0 & \text { on } \partial \Omega_{F}, \\ \mathbb{T}\left(V_{0}, Q_{0}\right) n=0 & \text { on } \partial \Omega_{S} .\end{cases}
$$

We also remark that $Q_{0}$ is characterized as the unique solution of the elliptic system

$$
\begin{cases}\Delta Q_{0}=-\nabla V_{0}:\left(\nabla v_{0}\right)^{*} & \text { in } \Omega_{F}, \\ Q_{0}=2 \varepsilon\left(V_{0}\right) n \cdot n & \text { on } \partial \Omega_{S}, \\ \frac{\partial Q_{0}}{\partial n}=\Delta V_{0} \cdot n+G_{0} \cdot n & \text { on } \partial \Omega .\end{cases}
$$

In the main result of Section 2, we will prove that, with these hypotheses on the data, system (1.14)1.22 admits a unique regular solution defined locally in time:

Proposition 2.1. Let $\widehat{X} \in B_{M}$ be given. There exist $\alpha>0$ and $C>0$ such that for all $T$ satisfying

$$
T^{\alpha}(1+M) \leqslant C
$$

for all $\left(V_{0}, \Xi_{1}, G\right)$ satisfying (2.1)-2.4, the system 1.14-1.22 admits a unique solution $(X, v, q, \xi)$ satisfying

$$
\begin{gathered}
v \in H^{2}\left(L^{2}\left(\Omega_{F}\right)\right) \cap H^{1}\left(H^{2}\left(\Omega_{F}\right)\right) \cap C^{1}\left(H^{1}\left(\Omega_{F}\right)\right) \cap L^{2}\left(H^{5 / 2+1 / 8}\left(\Omega_{F}\right)\right) \\
q \in H^{1}\left(H^{1}\left(\Omega_{F}\right)\right) \cap L^{2}\left(H^{3 / 2+1 / 8}\left(\Omega_{F}\right)\right)
\end{gathered}
$$

and

$$
\xi \in C^{2}\left(H^{1 / 2+1 / 8}\left(\Omega_{S}\right)\right) \cap C^{1}\left(H^{3 / 2+1 / 8}\left(\Omega_{S}\right)\right) \cap C^{0}\left(H^{5 / 2+1 / 8}\left(\Omega_{S}\right)\right) .
$$

The proof of Proposition 2.1 is based on a fixed point argument. We set

$$
\mathcal{Y}:=\left\{(\widetilde{v}, \widetilde{q}) \in \mathcal{Y}_{1} \times \mathcal{Y}_{2}: \widetilde{v}(0, \cdot)=V_{0}, \widetilde{q}(0, \cdot)=Q_{0} \text { and } \partial_{t} \widetilde{v}(0, \cdot)=V_{1} \text { in } \Omega_{F}\right\},
$$

where

$$
\mathcal{Y}_{1}:=H^{2}\left(L^{2}\left(\Omega_{F}\right)\right) \cap H^{1}\left(H^{2}\left(\Omega_{F}\right)\right) \cap C^{1}\left(H^{1}\left(\Omega_{F}\right)\right) \cap L^{2}\left(H^{5 / 2+1 / 8}\left(\Omega_{F}\right)\right)
$$

and

$$
\mathcal{Y}_{2}:=\left\{\widetilde{q} \in H^{1}\left(H^{1}\left(\Omega_{F}\right)\right) \cap L^{2}\left(H^{3 / 2+1 / 8}\left(\Omega_{F}\right)\right): \partial_{t} \widetilde{q} \in H^{1 / 4}\left(L^{2}\left(\partial \Omega_{S}\right)\right)\right\} .
$$

In what follows, we use the norms

$$
\begin{gathered}
\|\widetilde{v}\|_{\mathcal{Y}_{1}}:=\|\widetilde{v}\|_{H^{2}\left(L^{2}\left(\Omega_{F}\right)\right) \cap H^{1}\left(H^{2}\left(\Omega_{F}\right)\right) \cap L^{2}\left(H^{5 / 2+1 / 8}\left(\Omega_{F}\right)\right)}, \\
\|\widetilde{q}\|_{\mathcal{Y}_{2}}:=\|\widetilde{q}\|_{H^{1}\left(H^{1}\left(\Omega_{F}\right)\right) \cap L^{2}\left(H^{3 / 2+1 / 8}\left(\Omega_{F}\right)\right)}+\left\|\partial_{t} \widetilde{q}\right\|_{H^{1 / 4}\left(L^{2}\left(\partial \Omega_{S}\right)\right)}
\end{gathered}
$$

For any $(\widetilde{v}, \widetilde{q})$ given in $\mathcal{Y}$, we introduce the following auxiliary systems

$$
\begin{cases}\partial_{t t} \xi-\operatorname{div} \Sigma(\xi)=0 & \text { in }(0, T) \times \Omega_{S}, \\ \xi(t, \cdot)=\int_{0}^{t} \widetilde{v}(s, \cdot) d s & \text { on }(0, T) \times \partial \Omega_{S} \\ \xi(0, \cdot)=0, \quad \partial_{t} \xi(0, \cdot)=\Xi_{1} & \text { in } \Omega_{S}\end{cases}
$$


and

$$
\begin{cases}\partial_{t} v-\operatorname{div} \mathbb{T}(v, q)=F_{1} & \text { in }(0, T) \times \Omega_{F}, \\ \operatorname{div} v=F_{2} & \text { in }(0, T) \times \Omega_{F}, \\ \mathbb{T}(v, q) n=\Sigma(\xi) n+F_{3} & \text { on }(0, T) \times \partial \Omega_{S}, \\ v=0 & \text { on }(0, T) \times \partial \Omega, \\ v(0, \cdot)=V_{0} & \text { in } \Omega_{F},\end{cases}
$$

where

$$
\begin{gathered}
F_{1}:=G+\operatorname{div} \mathbb{T}_{\widehat{X}}(\widetilde{v}, \widetilde{q})-\operatorname{div} \mathbb{T}(\widetilde{v}, \widetilde{q}), \\
F_{2}:=-\nabla \widetilde{v}(t, y):\left[\operatorname{Cof}(\nabla \widehat{X})-I_{3}\right], \\
F_{3}:=-\mathbb{T}_{\widehat{X}}(\widetilde{v}, \widetilde{q}) n+\mathbb{T}(\widetilde{v}, \widetilde{q}) n .
\end{gathered}
$$

We recall that $\mathbb{T}_{\widehat{X}}$ is defined by $(1.6)$. Let us briefly explain why, in the space $\mathcal{Y}_{2}$, we add the fact that $\partial_{t} \widetilde{q} \in H^{1 / 4}\left(L^{2}\left(\partial \Omega_{S}\right)\right)$ : this will allow to get that $F_{3}$ belongs to $H^{5 / 4}\left(L^{2}\left(\partial \Omega_{S}\right)\right)$ (see $\left.\sqrt{2.43}\right)$ and it will be useful in the third step of subsection 2.4 to apply a regularity result from [15].

Let us now consider the map

$$
\Lambda: \mathcal{Y} \rightarrow \mathcal{Y}, \quad \Lambda(\widetilde{v}, \widetilde{q}):=(v, q),
$$

where $(v, q, \xi)$ is the solution of $2.8-2.9$.

In order to prove Proposition 2.1 we will show the two following properties:

1. $\Lambda$ is well-defined:

$$
\Lambda(\widetilde{v}, \widetilde{q}) \in \mathcal{Y} \quad \text { for all }(\widetilde{v}, \widetilde{q}) \in \mathcal{Y}
$$

2. There exist $\alpha>0$ and $C>0$ such that, for all $T$ satisfying $(2.6), \Lambda$ is a contraction.

This will imply that $\Lambda$ admits a unique fixed point and this will yield Proposition 2.1. To prove the second point, since $\Lambda$ is an affine function, it is sufficient to prove that

$$
\|(v, q)\|_{\mathcal{Y}} \leqslant C T^{\alpha} M\|(\widetilde{v}, \widetilde{q})\|_{\mathcal{Y}}
$$

under the condition that the data are null:

$$
\Xi_{1}=0, \quad V_{0}=0, \quad Q_{0}=0, \quad V_{1}=0, \quad G=0 .
$$

To prove the regularity 2.14 and the estimate 2.15, we follow similar arguments: in what follows, we will show simultaneously that $(v, q) \in \mathcal{Y}$ and that it satisfies the corresponding estimate when (2.16) holds.

To make the proof easier to read, we have separated it in different subsections: in subsection 2.1 we give estimates on terms involving $\widehat{X}$ and in subsection 2.2 , we estimate terms involving $(\widetilde{v}, \widetilde{q})$. These preliminary estimates are then used in subsection 2.3 to estimate the right-hand sides $F_{1}, F_{2}, F_{3}$ in 2.8 2.9. At last, subsection 2.1 resumes these different results and gives the proof of Proposition 2.1 It relies on regularity results for the two sub-problems: a hidden regularity result presented in [25] is applied to the linearized elasticity equation whereas a regularity result presented in [15] is applied to a nonstationary Stokes system.

\subsection{Estimates on the terms coming from $\widehat{X}$}

Using that $\widehat{X} \in B_{M}$ (see 1.11 for the definition of $B_{M}$ ), we first deduce from a standard Sobolev embedding that

$$
\begin{aligned}
\left\|\nabla \widehat{X}-I_{3}\right\|_{C^{0}\left([0, T] \times \overline{\Omega_{F}}\right)} \leqslant C\left\|\nabla \widehat{X}-I_{3}\right\|_{C^{0}\left(H^{3 / 2+1 / 8}\left(\Omega_{F}\right)\right)} & \\
\leqslant C T^{1 / 2}\|\widehat{X}\|_{H^{1}\left(H^{5 / 2+1 / 8}\left(\Omega_{F}\right)\right)} & \leqslant C T^{1 / 2} M .
\end{aligned}
$$

In particular, if

$$
T^{1 / 2} M \leqslant 1,
$$

then

$$
\left\{\begin{array}{l}
\|\nabla \widehat{X}\|_{C^{0}\left([0, T] \times \overline{\Omega_{F}}\right)} \leqslant C, \quad\|\operatorname{Cof}(\nabla \widehat{X})\|_{C^{0}\left([0, T] \times \overline{\Omega_{F}}\right)} \leqslant C, \\
\|\nabla \widehat{X}\|_{C^{0}\left(H^{3 / 2+1 / 8}\left(\Omega_{F}\right)\right)} \leqslant C, \quad\|\operatorname{Cof}(\nabla \widehat{X})\|_{C^{0}\left(H^{3 / 2+1 / 8}\left(\Omega_{F}\right)\right)} \leqslant C .
\end{array}\right.
$$


The above estimate and 2.17 yield

$$
\left\|\operatorname{Cof}(\nabla \widehat{X})-I_{3}\right\|_{C^{0}\left([0, T] \times \overline{\Omega_{F}}\right)} \leqslant C\left\|\operatorname{Cof}(\nabla \widehat{X})-I_{3}\right\|_{C^{0}\left(H^{3 / 2+1 / 8}\left(\Omega_{F}\right)\right)} \leqslant C T^{1 / 2} M .
$$

Since

$$
\|\widehat{v}\|_{\mathcal{S}_{2, T}} \leqslant M
$$

we can apply Lemma A.6 (with $p_{1}=+\infty, p_{2}=2, m_{1}=0, m_{2}=5 / 8$ and $\theta=1 / 5$ ) and get

$$
\left\|\nabla^{2} \widehat{v}\right\|_{L^{5 / 2}\left(H^{1 / 2}\left(\Omega_{F}\right)\right)} \leqslant C M \text {. }
$$

Gathering this estimate with

$$
\nabla^{2} \widehat{X}(t, y)=\int_{0}^{t} \nabla^{2} \widehat{v}(s, y) d s
$$

we deduce that

$$
\left\|\nabla^{2} \widehat{X}\right\|_{C^{0}\left(L^{3}\left(\Omega_{F}\right)\right)} \leqslant C T^{3 / 5} M
$$

Combining this estimate with 2.19 , we deduce

$$
\|\nabla \operatorname{Cof}(\nabla \widehat{X})\|_{C^{0}\left(L^{3}\left(\Omega_{F}\right)\right)} \leqslant C T^{3 / 5} M
$$

Using 2.19), 2.21 and the Sobolev embedding $H^{1+1 / 8}\left(\partial \Omega_{S}\right) \subset C^{0}\left(\partial \Omega_{S}\right)$, we deduce

$$
\left\|\left[\operatorname{Cof}(\nabla \widehat{X})-I_{3}\right]_{i_{1}, i_{2}}\right\|_{H^{1}\left(C^{0}\left(\partial \Omega_{S}\right)\right)}+\left\|\left[\operatorname{Cof}(\nabla \widehat{X})-I_{3}\right]_{i_{1}, i_{2}}[\operatorname{Cof}(\nabla \widehat{X})]_{i_{3}, i_{4}}\right\|_{H^{1}\left(C^{0}\left(\partial \Omega_{S}\right)\right)} \leqslant C M
$$

for all $i_{1}, \ldots, i_{4} \in\{1,2,3\}$. Now, for $p_{0} \in[2,5 / 2)$, we have from a Sobolev embedding and from Lemma A.6 (with $p_{1}=2, p_{2}=+\infty, m_{1}=13 / 8, m_{2}=1$ and $\theta=2 / p_{0}$ ):

$$
\begin{aligned}
& \|\nabla \widehat{v}\|_{L^{p_{0}}\left(L^{\infty}\left(\Omega_{F}\right)\right)} \leqslant C\|\nabla \widehat{v}\|_{L^{p_{0}\left(H^{1+5 /\left(4 p_{0}\right)}\left(\Omega_{F}\right)\right)}} \\
& \leqslant C\|\nabla \widehat{v}\|_{L^{2}\left(H^{13 / 8}\left(\Omega_{F}\right)\right)}^{2 / p_{0}}\|\nabla \widehat{v}\|_{C^{0}\left(H^{1}\left(\Omega_{F}\right)\right)}^{1-2 / p_{0}} \leqslant C M .
\end{aligned}
$$

Now we can obtain several estimates on the time derivatives of $\operatorname{Cof}(\nabla \widehat{X})$. Using the formula for the cofactor matrix and 2.19, 2.21 , we have

$$
\partial_{t} \operatorname{Cof}(\nabla \widehat{X}) \in C^{0}\left(H^{1}\left(\Omega_{F}\right)\right), \quad\left\|\partial_{t} \operatorname{Cof}(\nabla \widehat{X})\right\|_{\left.C^{0}\left(H^{1}\left(\Omega_{F}\right)\right)\right)} \leqslant C M
$$

and

$$
\partial_{t t} \operatorname{Cof}(\nabla \widehat{X}) \in L^{2}\left(L^{2}\left(\Omega_{F}\right)\right), \quad\left\|\partial_{t t} \operatorname{Cof}(\nabla \widehat{X})\right\|_{\left.L^{2}\left(L^{2}\left(\Omega_{F}\right)\right)\right)} \leqslant C M
$$

Finally, combining 2.19, 2.22, 2.23 and 2.26, we deduce

$$
\partial_{t} \nabla \operatorname{Cof}(\nabla \widehat{X}) \in L^{2}\left(L^{3}\left(\Omega_{F}\right)\right), \quad\left\|\partial_{t} \nabla \operatorname{Cof}(\nabla \widehat{X})\right\|_{\left.L^{2}\left(L^{3}\left(\Omega_{F}\right)\right)\right)} \leqslant C M T^{1 / 10} .
$$

We now estimate terms of the form

$$
z:=[\operatorname{Cof}(\nabla \widehat{X})]_{i_{1}, i_{2}} \frac{\partial \widehat{v}_{i_{3}}}{\partial y_{i_{4}}} \frac{\partial \widehat{X}_{i_{5}}}{\partial y_{i_{6}}}
$$

for some $i_{1}, \ldots, i_{6} \in\{1,2,3\}$. First using 2.19, 2.21 and 2.23, we notice that

$$
z \in C^{0}\left(H^{1}\left(\Omega_{F}\right)\right), \quad\|z\|_{\left.C^{0}\left(H^{1}\left(\Omega_{F}\right)\right)\right)} \leqslant C M .
$$

From 2.19 and 2.21, we also obtain

We now show that

$$
\begin{gathered}
z \in L^{2}\left(H^{3 / 2+1 / 8}\left(\Omega_{F}\right)\right), \quad\|z\|_{L^{2}\left(H^{3 / 2+1 / 8}\left(\Omega_{F}\right)\right)} \leqslant C M, \\
z \in H^{1}\left(L^{2}\left(\Omega_{F}\right)\right), \quad\|z\|_{H^{1}\left(L^{2}\left(\Omega_{F}\right)\right)} \leqslant C M .
\end{gathered}
$$

$$
z \in H^{1 / 4}\left(L^{4}\left(\partial \Omega_{S}\right)\right)
$$


Using the notation $\lfloor\cdot\rfloor_{s, 2,(0, T), \mathcal{X}}$ defined in A.2, the trace theorem and Lemma A.5 for $s=1 / 3, m_{1}=0$ and $m_{2}=3 / 2$ we deduce

$$
\begin{aligned}
& \|z\|_{H^{1 / 4}\left(L^{4}\left(\partial \Omega_{S}\right)\right)}^{2}=\|z\|_{L^{2}\left(L^{4}\left(\partial \Omega_{S}\right)\right)}^{2}+\lfloor z\rfloor_{1 / 4,2,(0, T), L^{4}\left(\partial \Omega_{S}\right)}^{2} \\
& \leqslant C\left(T\|z\|_{C^{0}\left(H^{1}\left(\Omega_{F}\right)\right)}^{2}+T^{1 / 6}\lfloor z\rfloor_{1 / 3,2,(0, T), L^{4}\left(\partial \Omega_{S}\right)}^{2}\right) \\
& \leqslant C\left(T\|z\|_{C^{0}\left(H^{1}\left(\Omega_{F}\right)\right)}^{2}+T^{1 / 6}\|z\|_{H^{1 / 3}\left(H^{1}\left(\Omega_{F}\right)\right)}^{2}\right) \\
& \leqslant C\left(T\|z\|_{C^{0}\left(H^{1}\left(\Omega_{F}\right)\right)}^{2}+T^{1 / 6}\|z\|_{L^{2}\left(H^{3 / 2}\left(\Omega_{F}\right)\right)}^{4 / 3}\|z\|_{H^{1}\left(L^{2}\left(\Omega_{F}\right)\right)}^{2 / 3}\right) .
\end{aligned}
$$

In particular, we have obtained

$$
\left\|[\operatorname{Cof}(\nabla \widehat{X})]_{i_{1}, i_{2}} \frac{\partial \widehat{v}_{i_{3}}}{\partial y_{i_{4}}} \frac{\partial \widehat{X}_{i_{5}}}{\partial y_{i_{6}}}\right\|_{H^{1 / 4}\left(L^{4}\left(\partial \Omega_{S}\right)\right)} \leqslant C T^{1 / 12} M
$$

for all $i_{1}, \ldots, i_{6} \in\{1,2,3\}$.

\subsection{Estimates on the terms coming from $(\widetilde{v}, \widetilde{q})$}

We recall that $(\widetilde{v}, \widetilde{q}) \in \mathcal{Y}$, where $\mathcal{Y}$ is defined by $(2.7)$. This yields other regularity properties and some estimates in the case of null data 2.16. First, we have

$$
\widetilde{v} \in C^{0}\left(H^{2}\left(\Omega_{F}\right)\right), \quad \widetilde{q} \in C^{0}\left(H^{1}\left(\Omega_{F}\right)\right)
$$

and if $\widetilde{v}(0, \cdot)=0$ and $\widetilde{q}(0, \cdot)=0$, then

$$
\|\widetilde{v}\|_{C^{0}\left(H^{2}\left(\Omega_{F}\right)\right)} \leqslant T^{1 / 2}\|\widetilde{v}\|_{H^{1}\left(H^{2}\left(\Omega_{F}\right)\right)}, \quad\|\widetilde{q}\|_{C^{0}\left(H^{1}\left(\Omega_{F}\right)\right)} \leqslant T^{1 / 2}\|\widetilde{q}\|_{H^{1}\left(H^{1}\left(\Omega_{F}\right)\right)} .
$$

We also have

$$
\partial_{t} \nabla \widetilde{v} \in C^{0}\left(L^{2}\left(\Omega_{F}\right)\right), \quad \nabla \widetilde{v} \in C^{0}\left(L^{6}\left(\Omega_{F}\right)\right)
$$

and if $\widetilde{v}(0, \cdot)=0$ and $\partial_{t} \widetilde{v}(0, \cdot)=0$, then

$$
\left\|\partial_{t} \nabla \widetilde{v}\right\|_{C^{0}\left(L^{2}\left(\Omega_{F}\right)\right)} \leqslant C\left(\|\widetilde{v}\|_{H^{1}\left(H^{2}\left(\Omega_{F}\right)\right)}+\|\widetilde{v}\|_{H^{2}\left(L^{2}\left(\Omega_{F}\right)\right)}\right)
$$

and

$$
\|\nabla \widetilde{v}\|_{C^{0}\left(L^{6}\left(\Omega_{F}\right)\right)} \leqslant C T^{1 / 2}\left(\|\widetilde{v}\|_{H^{1}\left(H^{2}\left(\Omega_{F}\right)\right)}+\|\widetilde{v}\|_{H^{2}\left(L^{2}\left(\Omega_{F}\right)\right)}\right) .
$$

Using the trace theorem, we also have $\nabla \widetilde{v} \in H^{1}\left(L^{4}\left(\partial \Omega_{S}\right)\right)$ with

$$
\|\nabla \widetilde{v}\|_{H^{1}\left(L^{4}\left(\partial \Omega_{S}\right)\right)} \leqslant C\|\widetilde{v}\|_{H^{1}\left(H^{2}\left(\Omega_{F}\right)\right)} .
$$

Using Lemma A.5, we have

$$
\begin{gathered}
\widetilde{v} \in H^{3 / 2+1 / 8}\left((0, T) \times \partial \Omega_{S}\right), \\
\|\widetilde{v}\|_{H^{7 / 4-1 / 16}\left(L^{2}\left(\partial \Omega_{S}\right)\right)} \leqslant C\left(\|\widetilde{v}\|_{H^{1}\left(H^{2}\left(\Omega_{F}\right)\right)}+\|\widetilde{v}\|_{H^{2}\left(L^{2}\left(\Omega_{F}\right)\right)}\right)
\end{gathered}
$$

and

$$
\|\widetilde{v}\|_{H^{4 / 5}\left(H^{3 / 2+1 / 8}\left(\partial \Omega_{S}\right)\right)} \leqslant C\left(\|\widetilde{v}\|_{H^{1}\left(H^{2}\left(\Omega_{F}\right)\right)}+\|\widetilde{v}\|_{L^{2}\left(H^{5 / 2+1 / 8}\left(\Omega_{F}\right)\right)}\right) .
$$

Moreover, according to Corollary A.3 if we assume that the data are null (2.16), we obtain

$$
\|\widetilde{v}\|_{H^{3 / 2+1 / 8}\left(L^{2}\left(\partial \Omega_{S}\right)\right)} \leqslant C T^{1 / 16}\|\tilde{v}\|_{H^{7 / 4-1 / 16}\left(L^{2}\left(\partial \Omega_{S}\right)\right)}
$$

and

which implies

$$
\|\widetilde{v}\|_{L^{2}\left(H^{3 / 2+1 / 8}\left(\partial \Omega_{S}\right)\right)} \leqslant C T^{4 / 5}\|\widetilde{v}\|_{H^{4 / 5}\left(H^{3 / 2+1 / 8}\left(\partial \Omega_{S}\right)\right)}
$$

$$
\|\widetilde{v}\|_{H^{3 / 2+1 / 8}\left((0, T) \times \partial \Omega_{S}\right)} \leqslant C T^{1 / 16}\|\widetilde{v}\|_{\mathcal{Y}_{1}} .
$$




\subsection{Estimates on $F_{1}, F_{2}, F_{3}$}

We prove here the following result

Lemma 2.2. Assume $(\widetilde{v}, \widetilde{q}) \in \mathcal{Y}$, where $\mathcal{Y}$ is defined by 2.7). Then the functions $F_{1}, F_{2}$ and $F_{3}$ given by 2.10, 2.11 and 2.12 satisfy

$$
\begin{gathered}
F_{1} \in L^{2}\left(H^{1 / 2+1 / 8}\left(\Omega_{F}\right)\right) \cap H^{1}\left(L^{2}\left(\Omega_{F}\right)\right), \\
F_{2} \in L^{2}\left(H^{3 / 2+1 / 8}\left(\Omega_{F}\right)\right) \cap H^{1}\left(H^{1}\left(\Omega_{F}\right)\right) \cap H^{2}\left(H^{-1}\left(\Omega_{F}\right)\right), \\
F_{3} \in L^{2}\left(H^{1+1 / 8}\left(\partial \Omega_{S}\right)\right) \cap H^{1}\left(H^{1 / 2}\left(\partial \Omega_{S}\right)\right) \cap H^{5 / 4}\left(L^{2}\left(\partial \Omega_{S}\right)\right),
\end{gathered}
$$

Assume in addition that we are in the case of null data (2.16). Then we have the following estimates

$$
\left\|F_{1}\right\|_{L^{2}\left(H^{1 / 2+1 / 8}\left(\Omega_{F}\right)\right)}+\left\|F_{2}\right\|_{L^{2}\left(H^{3 / 2+1 / 8}\left(\Omega_{F}\right)\right)}+\left\|F_{3}\right\|_{L^{2}\left(H^{1+1 / 8}\left(\partial \Omega_{S}\right)\right)} \leqslant C T^{1 / 2} M\|(\widetilde{v}, \widetilde{q})\| \mathcal{Y},
$$

and

$$
\begin{aligned}
& \left\|\partial_{t} F_{1}\right\|_{L^{2}\left(L^{2}\left(\Omega_{F}\right)\right)}+\left\|\partial_{t} F_{2}\right\|_{L^{2}\left(H^{1}\left(\Omega_{F}\right)\right) \cap H^{1}\left(H^{-1}\left(\Omega_{F}\right)\right)} \\
& +\left\|\partial_{t} F_{3}\right\|_{L^{2}\left(H^{1 / 2}\left(\partial \Omega_{S}\right)\right) \cap H^{1 / 4}\left(L^{2}\left(\partial \Omega_{S}\right)\right)} \leqslant C T^{1 / 4} M\|(\widetilde{v}, \widetilde{q})\| \mathcal{Y} .
\end{aligned}
$$

Proof. To avoid repeating the same arguments, we prove directly the estimates if the condition of null data 2.16 holds and we skip the proof of 2.41 -2.43 which follows the same process.

Combining 2.10, 2.17), 2.19), 2.22), 22.23) and 2.26) (with $p_{0}=9 / 4$ ), we deduce $\partial_{t} F_{1} \in$ $L^{2}\left(L^{2}\left(\Omega_{F}\right)\right)$ with

Now, from 2.11, we deduce

$$
\left\|\partial_{t} F_{1}\right\|_{L^{2}\left(L^{2}\left(\Omega_{F}\right)\right)} \leqslant C T^{1 / 2} M\|(\widetilde{v}, \widetilde{q})\|_{\mathcal{Y}}
$$

$$
\partial_{t} F_{2}=-\nabla \partial_{t} \widetilde{v}(t, y):\left[\operatorname{Cof}(\nabla \widehat{X})-I_{3}\right]-\nabla \widetilde{v}(t, y): \partial_{t} \operatorname{Cof}(\nabla \widehat{X}),
$$

so that $\partial_{t} F_{2} \in L^{2}\left(H^{1}\left(\Omega_{F}\right)\right)$ with

$$
\left\|\partial_{t} F_{2}\right\|_{L^{2}\left(H^{1}\left(\Omega_{F}\right)\right)} \leqslant C T^{1 / 2} M\|(\widetilde{v}, \widetilde{q})\|_{\mathcal{Y}}
$$

We also deduce that $\partial_{t t} F_{2}$ can be written as

$$
\partial_{t t} F_{2}=-\nabla \partial_{t t} \widetilde{v}(t, y):\left[\operatorname{Cof}(\nabla \widehat{X})-I_{3}\right]-2 \nabla \partial_{t} \widetilde{v}(t, y): \partial_{t} \operatorname{Cof}(\nabla \widehat{X})-\nabla \widetilde{v}(t, y): \partial_{t t} \operatorname{Cof}(\nabla \widehat{X}) .
$$

The term

$$
\nabla \partial_{t t} \widetilde{v}(t, y):\left[\operatorname{Cof}(\nabla \widehat{X})-I_{3}\right]
$$

can be transformed as a linear combination of terms of the form

$$
\frac{\partial}{\partial y_{i_{1}}}\left(\partial_{t t} \widetilde{v}_{i_{2}}\left[\operatorname{Cof}(\nabla \widehat{X})-I_{3}\right]_{i_{3}, i_{4}}\right)-\left(\partial_{t t} \widetilde{v}_{i_{2}} \frac{\partial}{\partial y_{i_{1}}}[\operatorname{Cof}(\nabla \widehat{X})]_{i_{3}, i_{4}}\right),
$$

with $i_{1}, \ldots, i_{4} \in\{1,2,3\}$. Using 2.20, 2.24, 2.27, 2.36, 2.37 and the Sobolev embedding $L^{6 / 5}\left(\Omega_{F}\right) \subset H^{-1}\left(\Omega_{F}\right)$, we obtain $\partial_{t t} F_{2} \in L^{2}\left(H^{-1}\left(\Omega_{F}\right)\right)$ with

$$
\left\|\partial_{t} F_{2}\right\|_{H^{1}\left(H^{-1}\left(\Omega_{F}\right)\right)} \leqslant C T^{1 / 2} M\|(\widetilde{v}, \widetilde{q})\|_{\mathcal{Y}} .
$$

Finally, $\partial_{t} F_{3}$ is the linear combination of terms of the form

$$
\begin{gathered}
{\left[\operatorname{Cof}(\nabla \widehat{X})-I_{3}\right]_{i_{1}, i_{2}} \frac{\partial \widetilde{q}}{\partial t} n_{i_{3}}, \quad\left[\operatorname{Cof}(\nabla \widehat{X})-I_{3}\right]_{i_{1}, i_{2}}[\operatorname{Cof}(\nabla \widehat{X})]_{i_{3}, i_{4}} \frac{\partial^{2} \widetilde{v}_{i_{5}}}{\partial t \partial y_{i_{6}}} n_{i_{7}}} \\
{[\operatorname{Cof}(\nabla \widehat{X})]_{i_{1}, i_{2}} \frac{\partial \widehat{v}_{i_{3}}}{\partial y_{i_{4}}} \frac{\partial \widehat{X}_{i_{5}}}{\partial y_{i_{6}}} \frac{\partial \widetilde{v}_{i_{7}}}{\partial y_{i_{8}}} n_{i_{9}}, \quad[\operatorname{Cof}(\nabla \widehat{X})]_{i_{1}, i_{2}} \frac{\partial \widehat{v}_{i_{3}}}{\partial y_{i_{4}}} \frac{\partial \widehat{X}_{i_{5}}}{\partial y_{i_{6}}} \widetilde{q} n_{i_{7}}}
\end{gathered}
$$

with $i_{1}, \ldots, i_{9} \in\{1,2,3\}$. Thus $\partial_{t} F_{3} \in L^{2}\left(H^{1 / 2}\left(\partial \Omega_{S}\right)\right)$ with

$$
\left\|\partial_{t} F_{3}\right\|_{L^{2}\left(H^{1 / 2}\left(\partial \Omega_{S}\right)\right)} \leqslant C T^{1 / 2} M\|(\widetilde{v}, \widetilde{q})\|_{\mathcal{Y}} .
$$


Then we estimate $\partial_{t} F_{3}$ in $H^{1 / 4}\left(L^{2}\left(\partial \Omega_{S}\right)\right)$. Using 2.34, 2.38 and Lemma A.7 with $s=1 / 4, \sigma_{0}=1$, $\mathcal{X}_{1}=\mathcal{X}_{2}=L^{4}\left(\partial \Omega_{S}\right)$ and $\mathcal{X}_{3}=L^{2}\left(\partial \Omega_{S}\right)$, we deduce

$$
\left\|[\operatorname{Cof}(\nabla \widehat{X})]_{i_{1}, i_{2}} \frac{\partial \widehat{v}_{i_{3}}}{\partial y_{i_{4}}} \frac{\partial \widehat{X}_{i_{5}}}{\partial y_{i_{6}}} \frac{\partial \widetilde{v}_{i_{7}}}{\partial y_{i_{8}}}\right\|_{H^{1 / 4}\left(L^{2}\left(\partial \Omega_{S}\right)\right)} \leqslant C T^{1 / 4} M\|(\widetilde{v}, \widetilde{q})\|_{\mathcal{Y}}
$$

and a similar estimate for the last term in 2.51). In order to estimate the first term in (2.51), we use 2.7), 2.25 and Lemma A.7 with $s=1 / 4, \sigma_{0}=1, \mathcal{X}_{1}=L^{2}\left(\partial \Omega_{S}\right), \mathcal{X}_{2}=L^{\infty}\left(\partial \Omega_{S}\right)$ and $\mathcal{X}_{3}=L^{2}\left(\partial \Omega_{S}\right)$ :

$$
\begin{aligned}
& \left\|\left[\operatorname{Cof}(\nabla \widehat{X})-I_{3}\right]_{i_{1}, i_{2}} \partial_{t} \widetilde{q}\right\|_{H^{1 / 4}\left(L^{2}\left(\partial \Omega_{S}\right)\right)} \\
& \leqslant C T^{1 / 4}\left\|\operatorname{Cof}(\nabla \widehat{X})-I_{3}\right\|_{H^{1}\left(L^{\infty}\left(\partial \Omega_{S}\right)\right)}\left\|\partial_{t} \widetilde{q}\right\|_{H^{1 / 4}\left(L^{2}\left(\partial \Omega_{S}\right)\right)} \leqslant C T^{1 / 4} M\|(\widetilde{v}, \widetilde{q})\| \mathcal{Y} .
\end{aligned}
$$

The second term in 2.51 is treated using again Lemma A.7 with $s=1 / 4, \sigma_{0}=1, \mathcal{X}_{1}=L^{2}\left(\partial \Omega_{S}\right)$, $\mathcal{X}_{2}=L^{\infty}\left(\partial \Omega_{S}\right)$ and $\mathcal{\mathcal { X }}_{3}=L^{2}\left(\partial \Omega_{S}\right)$ :

$$
\begin{gathered}
\left\|\left[\operatorname{Cof}(\nabla \widehat{X})-I_{3}\right]_{i_{1}, i_{2}}[\operatorname{Cof}(\nabla \widehat{X})]_{i_{3}, i_{4}} \frac{\partial^{2} \widetilde{v}_{i_{5}}}{\partial t \partial y_{i_{6}}}\right\|_{H^{1 / 4}\left(L^{2}\left(\partial \Omega_{S}\right)\right)} \\
\leqslant C T^{1 / 4}\left\|\left[\operatorname{Cof}(\nabla \widehat{X})-I_{3}\right]_{i_{1}, i_{2}}[\operatorname{Cof}(\nabla \widehat{X})]_{i_{3}, i_{4}}\right\|_{H^{1}\left(L^{\infty}\left(\partial \Omega_{S}\right)\right)}\left\|\partial_{t} \nabla \widetilde{v}\right\|_{H^{1 / 4}\left(L^{2}\left(\partial \Omega_{S}\right)\right)} .
\end{gathered}
$$

Combining the above inequality with 2.25 and Lemma A.8 applied to $f:=\partial_{t} \widetilde{v}$, we obtain

$$
\left\|\left[\operatorname{Cof}(\nabla \widehat{X})-I_{3}\right]_{i_{1}, i_{2}}[\operatorname{Cof}(\nabla \widehat{X})]_{i_{3}, i_{4}} \frac{\partial^{2} \widetilde{v}_{i_{5}}}{\partial t \partial y_{i_{6}}}\right\|_{H^{1 / 4}\left(L^{2}\left(\partial \Omega_{S}\right)\right)} \leqslant C T^{1 / 4} M\|(\widetilde{v}, \widetilde{q})\|_{\mathcal{Y}} .
$$

We deduce $\partial_{t} F_{3} \in H^{1 / 4}\left(L^{2}\left(\partial \Omega_{S}\right)\right)$ with

$$
\left\|\partial_{t} F_{3}\right\|_{H^{1 / 4}\left(L^{2}\left(\partial \Omega_{S}\right)\right)} \leqslant C T^{1 / 4} M\|(\widetilde{v}, \widetilde{q})\| \mathcal{Y}
$$

The estimates of

$$
\left\|F_{1}\right\|_{L^{2}\left(H^{1 / 2+1 / 8}\left(\Omega_{F}\right)\right)}, \quad\left\|F_{2}\right\|_{L^{2}\left(H^{3 / 2+1 / 8}\left(\Omega_{F}\right)\right)}, \quad\left\|F_{3}\right\|_{L^{2}\left(H^{1+1 / 8}\left(\partial \Omega_{S}\right)\right)}
$$

are similar.

\subsection{Proof of Proposition 2.1}

We are now in position to prove Proposition 2.1. As explained after the statement of Proposition 2.1 . we will prove that the map $\Lambda$ (see (2.13) ) is well-defined (2.14) and satisfes (2.15). By this way, $\Lambda$ will be a contraction in $\mathcal{Y}$ if 2.6 holds for some $\alpha>0$ and $C>0$. The proof is split into four steps. The first step introduces a lifting for the divergence in order to get a divergence-free problem. A regularity result [25] is applied to the elasticity equation in the second step whereas a result on the regularity of the Stokes problem in [15] is applied in the third step. In the last step, additional spatial regularity is proved.

Step 1. Time derivative and lifting.

We consider the solution $w$ of the problem

$$
\begin{cases}\Delta w=\partial_{t} F_{2} & \text { in }(0, T) \times \Omega_{F} \\ w=0 & \text { on }(0, T) \times \partial \Omega_{F}\end{cases}
$$

Using standard elliptic estimates and 2.42, we obtain

$$
\|w\|_{H^{1}\left(H^{1}\left(\Omega_{F}\right)\right) \cap L^{2}\left(H^{3}\left(\Omega_{F}\right)\right)} \leqslant C\left\|\partial_{t} F_{2}\right\|_{H^{1}\left(H^{-1}\left(\Omega_{F}\right)\right) \cap L^{2}\left(H^{1}\left(\Omega_{F}\right)\right)},
$$

so that

$$
\|\nabla w\|_{H^{1}\left(L^{2}\left(\Omega_{F}\right)\right) \cap L^{2}\left(H^{2}\left(\Omega_{F}\right)\right)} \leqslant C\left\|\partial_{t} F_{2}\right\|_{H^{1}\left(H^{-1}\left(\Omega_{F}\right)\right) \cap L^{2}\left(H^{1}\left(\Omega_{F}\right)\right)} .
$$

Then, if we formally differentiate 2.8 and 2.9 with respect to time and we use $\nabla w$ to lift the divergence condition on $\partial_{t} v$, we get that $\Xi:=\partial_{t} \xi$ and $(V, Q):=\left(\partial_{t} v-\nabla w, \partial_{t} q\right)$ have to satisfy 


$$
\begin{cases}\partial_{t t} \Xi-\operatorname{div} \Sigma(\Xi)=0 & \text { in }(0, T) \times \Omega_{S}, \\ \Xi=\widetilde{v} & \text { on }(0, T) \times \partial \Omega_{S}, \\ \Xi(0, \cdot)=\Xi_{1}, \quad \partial_{t} \Xi(0, \cdot)=0 & \text { in } \Omega_{S}\end{cases}
$$

and

$$
\begin{cases}\partial_{t} V-\operatorname{div} \mathbb{T}(V, Q)=\partial_{t} F_{1}-\partial_{t} \nabla w+2 \operatorname{div} \varepsilon(\nabla w)=: \widetilde{F}_{1} & \text { in }(0, T) \times \Omega_{F}, \\ \operatorname{div} V=0 & \text { in }(0, T) \times \Omega_{F}, \\ \mathbb{T}(V, Q) n=\Sigma(\Xi) n+\partial_{t} F_{3}-2 \varepsilon(\nabla w) n=: \widetilde{F}_{3} & \text { on }(0, T) \times \partial \Omega_{S}, \\ V=-\nabla w=: \widetilde{F}_{4} & \text { on }(0, T) \times \partial \Omega, \\ V(0, \cdot)=G_{0}+\Delta V_{0}-\nabla Q_{0}-\nabla w(0, \cdot) & \text { in } \Omega_{F} .\end{cases}
$$

Step 2. Estimates on the elastic displacement.

According to standard results (we refer for instance to [26), system (2.57) admits a unique weak solution in $C^{0}\left(L^{2}\left(\Omega_{S}\right)\right) \cap C^{1}\left(H^{-1}\left(\Omega_{S}\right)\right)$. To get regularity on $\partial_{t} \xi$, we will use hidden regularity results (we refer to 21] for the wave equation and to 25] for the elasticity equation).

We use that the Dirichlet condition $\tilde{v}$ satisfies 2.39 . Note that

$$
\Xi(0, \cdot)=\Xi_{1} \in H^{3 / 2+1 / 8}\left(\Omega_{S}\right), \quad \partial_{t} \Xi(0, \cdot)=0 \in H^{1 / 2+1 / 8}\left(\Omega_{S}\right), \quad \Xi=\widetilde{v} \in H^{3 / 2+1 / 8}\left((0, T) \times \partial \Omega_{S}\right),
$$

and the compatibility conditions are satisfied since

$$
\widetilde{v}(0, \cdot)=V_{0}=\Xi_{1} \text { on } \partial \Omega_{S} \quad \text { and } \quad \partial_{t} \widetilde{v}(0, \cdot)=V_{1}=0 \text { on } \partial \Omega_{S}
$$

(see 2.7) and the fourth and sixth conditions in 2.4). By this way, according to Theorem 3.2 in [25] and interpolation arguments, we deduce that $\xi(t, \cdot):=\int_{0}^{t} \Xi(s, \cdot) d s$ satisfies

$$
\partial_{t} \xi \in C^{0}\left(H^{3 / 2+1 / 8}\left(\Omega_{S}\right)\right) \cap C^{1}\left(H^{1 / 2+1 / 8}\left(\Omega_{S}\right)\right)
$$

and

with the estimate

$$
\Sigma\left(\partial_{t} \xi\right) n \in H^{1 / 2+1 / 8}\left((0, T) \times \partial \Omega_{S}\right)
$$

$$
\begin{aligned}
\left\|\partial_{t t} \xi\right\|_{C^{0}\left(H^{1 / 2+1 / 8}\left(\Omega_{S}\right)\right)}+\left\|\partial_{t} \xi\right\|_{C^{0}\left(H^{3 / 2+1 / 8}\left(\Omega_{S}\right)\right)}+ & \left\|\Sigma\left(\partial_{t} \xi\right) n\right\|_{H^{1 / 2+1 / 8}\left((0, T) \times \partial \Omega_{S}\right)} \\
& \leqslant C\left(\left\|\Xi_{1}\right\|_{H^{3 / 2+1 / 8}\left(\Omega_{S}\right)}+\|\widetilde{v}\|_{H^{3 / 2+1 / 8}\left((0, T) \times \partial \Omega_{S}\right)}\right)
\end{aligned}
$$

where $C$ is independent of $T$. Moreover, in the case of null data 2.16, if we use 2.40, we deduce

$$
\left\|\partial_{t t} \xi\right\|_{C^{0}\left(H^{1 / 2+1 / 8}\left(\Omega_{S}\right)\right)}+\left\|\partial_{t} \xi\right\|_{C^{0}\left(H^{3 / 2+1 / 8}\left(\Omega_{S}\right)\right)}+\left\|\Sigma\left(\partial_{t} \xi\right) n\right\|_{H^{1 / 2+1 / 8}\left((0, T) \times \partial \Omega_{S}\right)} \leqslant C T^{1 / 16}\|\widetilde{v}\|_{\mathcal{y}_{1}} .
$$

Step 3. Estimates on the fluid velocity and pressure.

The existence and uniqueness of a solution of 2.58 will come from results in [15]. First we check that the compatibility conditions are satisfied: from the first and the third conditions in 2.4 , 2.63) and 2.55 , we deduce

$$
\operatorname{div}\left(G_{0}+\Delta V_{0}-\nabla Q_{0}-\nabla w(0, \cdot)\right)=0 \text { in } \Omega_{F} .
$$

Moreover, from the first and the sixth conditions in 2.4 and 2.58, we deduce

$$
G_{0}+\Delta V_{0}-\nabla Q_{0}-\nabla w(0, \cdot)=\widetilde{F}_{4}(0, \cdot) \quad \text { on } \partial \Omega .
$$

Finally, using (2.41)-2.43), 2.56), Lemma A.8 with $f:=\nabla w$, we have

$$
\widetilde{F}_{1} \in L^{2}\left(L^{2}\left(\Omega_{F}\right)\right), \quad \widetilde{F}_{3} \in L^{2}\left(H^{1 / 2}\left(\partial \Omega_{S}\right)\right) \cap H^{1 / 4}\left(L^{2}\left(\partial \Omega_{S}\right)\right),
$$

$$
\widetilde{F}_{4} \in L^{2}\left(H^{3 / 2}(\partial \Omega)\right) \cap H^{3 / 4}\left(L^{2}(\partial \Omega)\right) .
$$


Thus, according to [15] (Theorem 7.5 with $r=0$ ), the system 2.58) admits a unique strong solution $(V, Q)$ with the following regularity

$$
V \in L^{2}\left(H^{2}\left(\Omega_{F}\right)\right) \cap H^{1}\left(L^{2}\left(\Omega_{F}\right)\right) \cap C^{0}\left(H^{1}\left(\Omega_{F}\right)\right), \quad Q \in L^{2}\left(H^{1}\left(\Omega_{F}\right)\right) .
$$

Let us set

$$
v(t, \cdot):=V_{0}+\int_{0}^{t}(V(s, \cdot)+\nabla w(s, \cdot)) d s, \quad q(t, \cdot):=Q_{0}+\int_{0}^{t} Q(s, \cdot) d s .
$$

According to 2.4 and since

$$
F_{1}(0, \cdot)=G_{0}, \quad F_{2}(0, \cdot)=0 \quad F_{3}(0, \cdot)=0
$$

we see that $(v, q, \xi)$ is the solution of 2.8-2.9. Moreover, using again 2.56), we have

$$
v \in H^{1}\left(H^{2}\left(\Omega_{F}\right)\right) \cap H^{2}\left(L^{2}\left(\Omega_{F}\right)\right) \cap C^{1}\left(H^{1}\left(\Omega_{F}\right)\right), \quad q \in H^{1}\left(H^{1}\left(\Omega_{F}\right)\right) .
$$

In the case of null data 2.16, we have the estimate

$$
\begin{gathered}
\|v\|_{H^{1}\left(H^{2}\left(\Omega_{F}\right)\right) \cap H^{2}\left(L^{2}\left(\Omega_{F}\right)\right) \cap C^{1}\left(H^{1}\left(\Omega_{F}\right)\right)}+\|q\|_{H^{1}\left(H^{1}\left(\Omega_{F}\right)\right)} \\
\leqslant C\left(\left\|\partial_{t} F_{1}\right\|_{L^{2}\left(L^{2}\left(\Omega_{F}\right)\right)}+\left\|\partial_{t} F_{2}\right\|_{H^{1}\left(H^{-1}\left(\Omega_{F}\right)\right) \cap L^{2}\left(H^{1}\left(\Omega_{F}\right)\right)}\right. \\
+\left\|\partial_{t} F_{3}\right\|_{L^{2}\left(H^{1 / 2}\left(\partial \Omega_{S}\right)\right) \cap H^{1 / 4}\left(L^{2}\left(\partial \Omega_{S}\right)\right)} \\
\left.\quad+\left\|\Sigma\left(\partial_{t} \xi\right) n\right\|_{L^{2}\left(H^{1 / 2}\left(\partial \Omega_{S}\right)\right) \cap H^{1 / 4}\left(L^{2}\left(\partial \Omega_{S}\right)\right)}\right) .
\end{gathered}
$$

We then use 2.45 to estimate the three first terms and 2.62 to estimate the last term in the right-hand side and we obtain

$$
\|v\|_{H^{1}\left(H^{2}\left(\Omega_{F}\right)\right) \cap H^{2}\left(L^{2}\left(\Omega_{F}\right)\right) \cap C^{1}\left(H^{1}\left(\Omega_{F}\right)\right)}+\|q\|_{H^{1}\left(H^{1}\left(\Omega_{F}\right)\right)} \leqslant C\left(T^{1 / 4} M+T^{1 / 16}\right)\|(\widetilde{v}, \widetilde{q})\|_{\mathcal{Y}} .
$$

In order to prove that

$$
\partial_{t} q \in H^{1 / 4}\left(L^{2}\left(\partial \Omega_{S}\right)\right)
$$

we use the boundary condition in 2.58. First, from Lemma A.8 applied to $f:=\partial_{t} v$ and 2.65, we find

$$
\partial_{t} \nabla v \in H^{1 / 4}\left(L^{2}\left(\partial \Omega_{S}\right)\right)
$$

with

$$
\left\|\partial_{t} \nabla v\right\|_{H^{1 / 4}\left(L^{2}\left(\partial \Omega_{S}\right)\right)} \leqslant C\left(T^{1 / 4} M+T^{1 / 16}\right)\|(\widetilde{v}, \widetilde{q})\| \mathcal{Y} .
$$

On the other hand, from 2.16, 2.62 and Corollary A.3

$$
\left\|\Sigma\left(\partial_{t} \xi\right) n\right\|_{H^{1 / 4}\left(L^{2}\left(\partial \Omega_{S}\right)\right)} \leqslant C T^{1 / 2}\|\widetilde{v}\|_{\mathcal{Y}_{1}}
$$

Using 2.45, we deduce 2.66 with

$$
\left\|\partial_{t} q\right\|_{H^{1 / 4}\left(L^{2}\left(\partial \Omega_{S}\right)\right)} \leqslant C\left(T^{1 / 4} M+T^{1 / 16}\right)\|(\widetilde{v}, \widetilde{q})\|_{\mathcal{Y}} .
$$

Step 4. Additional spatial regularity.

We still have to prove that $\xi \in C^{0}\left(H^{5 / 2+1 / 8}\left(\Omega_{S}\right)\right)$ and $(v, q) \in L^{2}\left(H^{5 / 2+1 / 8}\left(\Omega_{F}\right)\right) \times L^{2}\left(H^{3 / 2+1 / 8}\left(\Omega_{F}\right)\right)$.

We first consider 2.8 as an elliptic problem. For the boundary condition, according to (2.7), we have

$$
\xi \in C^{0}\left(H^{2+1 / 8}\left(\partial \Omega_{S}\right)\right)
$$

with

$$
\|\xi\|_{C^{0}\left(H^{2+1 / 8}\left(\partial \Omega_{S}\right)\right)} \leqslant C T^{1 / 2}\|\widetilde{v}\|_{L^{2}\left(H^{5 / 2+1 / 8}\left(\Omega_{F}\right)\right)} .
$$

Thus, according to 2.60, we deduce that

$$
\xi \in C^{0}\left(H^{5 / 2+1 / 8}\left(\Omega_{S}\right)\right)
$$

and in the case of null data 2.16, gathering 2.62, 2.68) and the trace theorem, we obtain

$$
\|\Sigma(\xi) n\|_{C^{0}\left(H^{1+1 / 8}\left(\partial \Omega_{S}\right)\right)} \leqslant C T^{1 / 8}\|\widetilde{v}\|_{\mathcal{Y}_{1}} .
$$


Then, we consider 2.9 as an elliptic system and we obtain

$$
\begin{aligned}
& \|v\|_{L^{2}\left(H^{5 / 2+1 / 8}\left(\Omega_{F}\right)\right)}+\|q\|_{L^{2}\left(H^{3 / 2+1 / 8}\left(\Omega_{F}\right)\right)} \\
& \quad \leqslant C\left(\|v\|_{H^{1}\left(H^{1 / 2+1 / 8}\left(\Omega_{F}\right)\right)}+\left\|F_{1}\right\|_{L^{2}\left(H^{1 / 2+1 / 8}\left(\Omega_{F}\right)\right)}\right. \\
& \left.\quad+\left\|F_{2}\right\|_{L^{2}\left(H^{3 / 2+1 / 8}\left(\Omega_{F}\right)\right)}+\left\|F_{3}\right\|_{L^{2}\left(H^{1+1 / 8}\left(\partial \Omega_{S}\right)\right)}+\|\Sigma(\xi) n\|_{L^{2}\left(H^{1+1 / 8}\left(\partial \Omega_{F}\right)\right)}\right) .
\end{aligned}
$$

Using 2.44, 2.65, 2.69 to estimate the right-hand side, we deduce

$$
\|v\|_{L^{2}\left(H^{5 / 2+1 / 8}\left(\Omega_{F}\right)\right)}+\|q\|_{L^{2}\left(H^{3 / 2+1 / 8}\left(\Omega_{F}\right)\right)} \leqslant C\left(T^{1 / 4} M+T^{1 / 16}\right)\|(\widetilde{v}, \widetilde{q})\|_{\mathcal{Y}}
$$

Gathering 2.65, 2.67) and 2.70 yields 2.15. This ends the proof of Proposition 2.1.

\section{Proof of Theorem 1.4}

Let us consider $\widehat{X} \in B_{M}$ (see 1.11 ). In order to prove Theorem 1.4 we first assume that the data $V_{0}, \Xi_{1}, G$ are smoother by replacing the hypotheses $\left.(1.23), 1.24\right)$ and $(1.25)$ by the stronger hypotheses (2.1)-(2.4). According to Proposition 2.1. system 1.14 - 1.22) admits a strong solution $(X, v, q, \xi)$ in $(0, T)$ where the existence time $T$ satisfies $(2.6)$. The regularity given by Proposition 2.1 will allow us to give a sense to all the terms appearing in the computations made in this section. The goal of the next subsections (from subsection 3.1 to subsection 3.5 is to prove the estimate (1.28) on $(X, v, q, \xi)$.

In subsection 3.1. we first prove an energy estimate satisfied by the solution. Next, in subsection 3.2 we consider the system satisfied by the time derivative of the solution and prove an energy estimate associated to this system. In subsection 3.3 we get spatial regularity thanks to standard elliptic estimates, more precisely, using the previous subsections, we estimate $\xi$ in $C^{0}\left(H^{2}\left(\Omega_{S}\right)\right)$ and $(v, q)$ in $C^{0}\left(H^{2}\left(\Omega_{F}\right)\right) \times$ $C^{0}\left(H^{1}\left(\Omega_{F}\right)\right)$. This leads to estimate 3.32 . Then, the tricky part of the proof consists in obtaining more regularity in space for the solution. To do so, some useful results on Stokes system are stated and proved in subsection 3.4 and the additional regularity results are given in subsection 3.5 At last, in subsection 3.6. we finish the proof by a density argument to deal with less regular data.

\subsection{Energy estimates}

We multiply 1.14 by $v$, integrate by parts and use $1.15,1.19$ and 1.20 :

$$
\begin{aligned}
\frac{1}{2} \frac{d}{d t} \int_{\Omega_{F}}|v|^{2} d y+\int_{\Omega_{F}}\left\{\left[(\nabla v) \operatorname{Cof}(\nabla \widehat{X})^{*}+\operatorname{Cof}(\nabla \widehat{X})(\nabla v)^{*}-q I_{3}\right] \operatorname{Cof}(\nabla \widehat{X})\right\}: \nabla v d y & \\
& +\int_{\partial \Omega_{S}} \Sigma(\xi) n \cdot \partial_{t} \xi d \gamma=\int_{\Omega_{F}} G \cdot v d y .
\end{aligned}
$$

We multiply 1.16 by $\partial_{t} \xi$ and integrate by parts:

$$
\frac{1}{2} \frac{d}{d t}\left(\int_{\Omega_{S}}\left[\left|\partial_{t} \xi\right|^{2}+2 \lambda_{1}|\varepsilon(\xi)|^{2}+\lambda_{2}(\operatorname{div} \xi)^{2}\right] d y\right)=\int_{\partial \Omega_{S}} \Sigma(\xi) n \cdot \partial_{t} \xi d \gamma .
$$

Adding the two above equations, we deduce

$$
\begin{aligned}
& \frac{1}{2} \frac{d}{d t} \int_{\Omega_{F}}|v|^{2} d y+\frac{1}{2} \int_{\Omega_{F}}|\varepsilon(v)|^{2} d y+\frac{1}{2} \frac{d}{d t}\left(\int_{\Omega_{S}}\left[\left|\partial_{t} \xi\right|^{2}+2 \lambda_{1}|\varepsilon(\xi)|^{2}+\lambda_{2}(\operatorname{div} \xi)^{2}\right] d y\right) \\
& \leqslant C\left\|\nabla \widehat{X}-I_{3}\right\|_{C^{0}\left([0, T] \times \overline{\Omega_{F}}\right)} \int_{\Omega_{F}}|\nabla v|^{2} d y+\left|\int_{\Omega_{F}} G \cdot v d y\right| .
\end{aligned}
$$

Using Korn's inequality (see for instance [16], 2.17) and $T^{1 / 2} M \leqslant C$ with $C$ small enough, we deduce

$$
\begin{aligned}
\|v\|_{C^{0}\left(L^{2}\left(\Omega_{F}\right)\right)}+\|v\|_{L^{2}\left(H^{1}\left(\Omega_{F}\right)\right)}+\|\xi\|_{C^{1}\left(L^{2}\left(\Omega_{S}\right)\right)} & +\|\xi\|_{C^{0}\left(H^{1}\left(\Omega_{S}\right)\right)} \\
\leqslant & C\left(\left\|V_{0}\right\|_{L^{2}\left(\Omega_{F}\right)}+\left\|\Xi_{1}\right\|_{L^{2}\left(\Omega_{S}\right)}+\|G\|_{L^{2}\left(L^{2}\left(\Omega_{F}\right)\right)}\right) .
\end{aligned}
$$




\subsection{Estimates on the time derivative}

We first differentiate $1.14-1.20$ with respect to time:

$$
\begin{aligned}
& \partial_{t t} v-\operatorname{div} \partial_{t} \mathbb{T}_{\widehat{X}}(v, q)=\partial_{t} G \quad \text { in }(0, T) \times \Omega_{F}, \\
& \left(\partial_{t} \nabla v\right): \operatorname{Cof}(\nabla \widehat{X})+\nabla v: \partial_{t} \operatorname{Cof}(\nabla \widehat{X})=0 \quad \text { in }(0, T) \times \Omega_{F} \text {, } \\
& \partial_{t t t} \xi-\operatorname{div} \Sigma\left(\partial_{t} \xi\right)=0 \quad \text { in }(0, T) \times \Omega_{S}, \\
& \partial_{t} v=0 \quad \text { on }(0, T) \times \partial \Omega, \\
& \partial_{t} v=\partial_{t t} \xi \quad \text { on }(0, T) \times \partial \Omega_{S}, \\
& \partial_{t} \mathbb{T}_{\widehat{X}}(v, q) n=\Sigma\left(\partial_{t} \xi\right) n \quad \text { on }(0, T) \times \partial \Omega_{S}, \\
& \begin{cases}\partial_{t} v(0, \cdot)=V_{1} & \text { in } \Omega_{F}, \\
\partial_{t} \xi(0, \cdot)=\Xi_{1}, \partial_{t t} \xi(0, \cdot)=0 & \text { in } \Omega_{S} .\end{cases}
\end{aligned}
$$

We recall that $\mathbb{T}_{\widehat{X}}$ is defined by 1.6 and that $V_{1}$ is introduced in 2.4. Applying standard elliptic results on 2.5 yields

$$
\left\|Q_{0}\right\|_{H^{1}\left(\Omega_{F}\right)} \leqslant C\left(1+\left\|v_{0}\right\|_{H^{2}\left(\Omega_{F}\right)}\right)\left\|V_{0}\right\|_{H^{2}\left(\Omega_{F}\right)}+C\left\|G_{0}\right\|_{L^{2}\left(\Omega_{F}\right)} .
$$

We thus deduce that $V_{1}$ satisfies

$$
\left\|V_{1}\right\|_{L^{2}\left(\Omega_{F}\right)} \leqslant C\left(1+\left\|v_{0}\right\|_{H^{2}\left(\Omega_{F}\right)}\right)\left\|V_{0}\right\|_{H^{2}\left(\Omega_{F}\right)}+C\left\|G_{0}\right\|_{L^{2}\left(\Omega_{F}\right)} .
$$

As for the proof of $(3.2)$, we multiply $(3.3)$ by $\partial_{t} v$ and $(3.5)$ by $\partial_{t t} \xi$, and after some integrations by parts, we find

$$
\begin{aligned}
\frac{1}{2} \frac{d}{d t} \int_{\Omega_{F}}\left|\partial_{t} v\right|^{2} d y & +\int_{\Omega_{F}} \partial_{t}\left\{\left[(\nabla v) \operatorname{Cof}(\nabla \widehat{X})^{*}+\operatorname{Cof}(\nabla \widehat{X})(\nabla v)^{*}-q I_{3}\right] \operatorname{Cof}(\nabla \widehat{X})\right\}: \partial_{t} \nabla v d y \\
& +\frac{1}{2} \frac{d}{d t}\left(\int_{\Omega_{S}}\left[\left|\partial_{t t} \xi\right|^{2}+2 \lambda_{1}\left|\varepsilon\left(\partial_{t} \xi\right)\right|^{2}+\lambda_{2}\left(\operatorname{div} \partial_{t} \xi\right)^{2}\right] d y\right)=\int_{\Omega_{F}} \partial_{t} G \cdot \partial_{t} v d y
\end{aligned}
$$

Now, we integrate this expression on the time interval $(0, t)$. For the second integral, we expand the first time derivative:

$$
\begin{aligned}
& \int_{0}^{t} \int_{\Omega_{F}} \partial_{t}\left\{\left[(\nabla v) \operatorname{Cof}(\nabla \widehat{X})^{*}+\operatorname{Cof}(\nabla \widehat{X})(\nabla v)^{*}-q I_{3}\right] \operatorname{Cof}(\nabla \widehat{X})\right\}: \partial_{t} \nabla v d y d s \\
& =\int_{0}^{t} \int_{\Omega_{F}}\left[\left(\partial_{t} \nabla v\right) \operatorname{Cof}(\nabla \widehat{X})^{*} \operatorname{Cof}(\nabla \widehat{X})+\operatorname{Cof}(\nabla \widehat{X})\left(\partial_{t} \nabla v\right)^{*} \operatorname{Cof}(\nabla \widehat{X})\right]: \partial_{t} \nabla v d y d s \\
& +\int_{0}^{t} \int_{\Omega_{F}}\left[(\nabla v) \partial_{t} \operatorname{Cof}(\nabla \widehat{X})^{*} \operatorname{Cof}(\nabla \widehat{X})+(\nabla v) \operatorname{Cof}(\nabla \widehat{X})^{*} \partial_{t} \operatorname{Cof}(\nabla \widehat{X})\right]: \partial_{t} \nabla v d y d s \\
& +\int_{0}^{t} \int_{\Omega_{F}}\left[\partial_{t} \operatorname{Cof}(\nabla \widehat{X})(\nabla v)^{*} \operatorname{Cof}(\nabla \widehat{X})+\operatorname{Cof}(\nabla \widehat{X})(\nabla v)^{*} \partial_{t} \operatorname{Cof}(\nabla \widehat{X})\right]: \partial_{t} \nabla v d y d s \\
& -\int_{0}^{t} \int_{\Omega_{F}} \partial_{t}[q \operatorname{Cof}(\nabla \widehat{X})]: \partial_{t} \nabla v d y d s .
\end{aligned}
$$

Using 2.17) and 2.19, the first integral gives:

$$
\begin{aligned}
& \int_{0}^{t} \int_{\Omega_{F}}\left[\left(\partial_{t} \nabla v\right) \operatorname{Cof}(\nabla \widehat{X})^{*} \operatorname{Cof}(\nabla \widehat{X})+\operatorname{Cof}(\nabla \widehat{X})\left(\partial_{t} \nabla v\right)^{*} \operatorname{Cof}(\nabla \widehat{X})\right]: \partial_{t} \nabla v d y d s \\
& \geqslant \int_{0}^{t} \int_{\Omega_{F}}\left|\varepsilon\left(\partial_{t} v\right)\right|^{2} d y d s-C \int_{0}^{T}\left\|\nabla \widehat{X}-I_{3}\right\|_{C^{0}\left([0, T] \times \overline{\Omega_{F}}\right)} \int_{\Omega_{F}}\left|\nabla \partial_{t} v\right|^{2} d y d s \\
& \geqslant \int_{0}^{t} \int_{\Omega_{F}}\left|\varepsilon\left(\partial_{t} v\right)\right|^{2} d y d s-C T^{1 / 2} M\|v\|_{H^{1}\left(H^{1}\left(\Omega_{F}\right)\right)}^{2} .
\end{aligned}
$$


Using 2.27) and $H^{1}\left(\Omega_{F}\right) \subset L^{6}\left(\Omega_{F}\right)$, we deduce for the second and third integrals of 3.13 :

$$
\begin{array}{r}
\left|\int_{0}^{t} \int_{\Omega_{F}}\left[(\nabla v) \partial_{t} \operatorname{Cof}(\nabla \widehat{X})^{*} \operatorname{Cof}(\nabla \widehat{X})+(\nabla v) \operatorname{Cof}(\nabla \widehat{X})^{*} \partial_{t} \operatorname{Cof}(\nabla \widehat{X})\right]: \partial_{t} \nabla v d y d s\right| \\
+\left|\int_{0}^{t} \int_{\Omega_{F}}\left[\partial_{t} \operatorname{Cof}(\nabla \widehat{X})(\nabla v)^{*} \operatorname{Cof}(\nabla \widehat{X})+\operatorname{Cof}(\nabla \widehat{X})(\nabla v)^{*} \partial_{t} \operatorname{Cof}(\nabla \widehat{X})\right]: \partial_{t} \nabla v d y d s\right| \\
\leqslant C_{\delta} T M^{2}\|v\|_{C^{0}\left(H^{2}\left(\Omega_{F}\right)\right)}^{2}+\delta\|v\|_{H^{1}\left(H^{1}\left(\Omega_{F}\right)\right)}^{2}
\end{array}
$$

for any $\delta>0$. For the last term in 3.13 , we have:

$$
\begin{aligned}
\int_{0}^{t} \int_{\Omega_{F}} \partial_{t}[q \operatorname{Cof}(\nabla \widehat{X})]: \partial_{t} \nabla v d y d s=\int_{0}^{t} \int_{\Omega_{F}} \partial_{t} q \operatorname{Cof}(\nabla \widehat{X}): \partial_{t} \nabla v d y d s & +\int_{0}^{t} \int_{\Omega_{F}} q \partial_{t}[\operatorname{Cof}(\nabla \widehat{X})]: \partial_{t} \nabla v d y d s
\end{aligned}
$$

Combining the above estimate and 3.4 , we deduce

$$
\begin{aligned}
& \int_{0}^{t} \int_{\Omega_{F}} \partial_{t}[q \operatorname{Cof}(\nabla \widehat{X})]: \partial_{t} \nabla v d y d s \\
& =-\int_{0}^{t} \int_{\Omega_{F}}\left(\partial_{t} q\right) \nabla v: \partial_{t} \operatorname{Cof}(\nabla \widehat{X}) d y d s+\int_{0}^{t} \int_{\Omega_{F}} q \partial_{t}[\operatorname{Cof}(\nabla \widehat{X})]: \partial_{t} \nabla v d y d s \\
& =-\int_{\Omega_{F}} q(t) \nabla v(t): \partial_{t} \operatorname{Cof}(\nabla \widehat{X})(t, \cdot) d y+\int_{\Omega_{F}} Q_{0} \nabla V_{0}:\left(\partial_{t} \operatorname{Cof}(\nabla \widehat{X})\right)(0, \cdot) d y \\
& +2 \int_{0}^{t} \int_{\Omega_{F}} q\left(\partial_{t} \nabla v\right): \partial_{t} \operatorname{Cof}(\nabla \widehat{X}) d y d s+\int_{0}^{t} \int_{\Omega_{F}} q \nabla v: \partial_{t t} \operatorname{Cof}(\nabla \widehat{X}) d y d s .
\end{aligned}
$$

For the first integral in the right-hand side, we have:

$$
\begin{aligned}
\left|\int_{\Omega_{F}} q(t) \nabla v(t): \partial_{t} \operatorname{Cof}(\nabla \widehat{X})(t, \cdot) d y\right| & \leqslant C\|q\|_{C^{0}\left(L^{6}\left(\Omega_{F}\right)\right)}\left\|\nabla v: \partial_{t} \operatorname{Cof}(\nabla \widehat{X})\right\|_{C^{0}\left(L^{6 / 5}\left(\Omega_{F}\right)\right)} \\
& \leqslant \delta\|q\|_{C^{0}\left(H^{1}\left(\Omega_{F}\right)\right)}^{2}+C_{\delta}\left\|\nabla v: \partial_{t} \operatorname{Cof}(\nabla \widehat{X})\right\|_{C^{0}\left(L^{6 / 5}\left(\Omega_{F}\right)\right)}^{2}
\end{aligned}
$$

for any $\delta>0$. We rewrite the last term:

$$
\nabla v: \partial_{t} \operatorname{Cof}(\nabla \widehat{X})=-\nabla V_{0}:\left(\nabla v_{0}\right)^{*}+\int_{0}^{t} \partial_{t}\left(\nabla v: \partial_{t} \operatorname{Cof}(\nabla \widehat{X})\right) d s
$$

where we have used that $\left(\partial_{t} \operatorname{Cof}(\nabla \widehat{X})\right)(0, \cdot)=-\left(\nabla v_{0}\right)^{*}$. Since

$$
\begin{aligned}
\left\|\int_{0}^{t} \partial_{t}\left(\nabla v: \partial_{t} \operatorname{Cof}(\nabla \widehat{X})\right) d s\right\|_{L^{6 / 5}\left(\Omega_{F}\right)} \leqslant C \int_{0}^{t}\left\|\partial_{t} \nabla v\right\|_{L^{2}\left(\Omega_{F}\right)}\left\|\partial_{t} \operatorname{Cof}(\nabla \widehat{X})\right\|_{L^{6}\left(\Omega_{F}\right)} d s & \\
& +C \int_{0}^{t}\|\nabla v\|_{L^{6}\left(\Omega_{F}\right)}\left\|\partial_{t t} \operatorname{Cof}(\nabla \widehat{X})\right\|_{L^{2}\left(\Omega_{F}\right)} d s
\end{aligned}
$$

we get from 2.27 and 2.28

$$
\left\|\int_{0}^{t} \partial_{t}\left(\nabla v: \partial_{t} \operatorname{Cof}(\nabla \widehat{X})\right) d s\right\|_{L^{6 / 5}\left(\Omega_{F}\right)} \leqslant C T^{1 / 2} M\|v\|_{H^{1}\left(H^{1}\left(\Omega_{F}\right)\right) \cap C^{0}\left(H^{2}\left(\Omega_{F}\right)\right)} .
$$

Gathering 3.17, 3.18 and the above equation, we obtain

$$
\begin{aligned}
\left|\int_{\Omega_{F}} q(t) \nabla v(t): \partial_{t} \operatorname{Cof}(\nabla \widehat{X})(t, \cdot) d y\right| \leqslant \delta\|q\|_{C^{0}\left(H^{1}\left(\Omega_{F}\right)\right)}^{2} & +C_{\delta}\left\|v_{0}\right\|_{H^{2}\left(\Omega_{F}\right)}^{2}\left\|V_{0}\right\|_{H^{2}\left(\Omega_{F}\right)}^{2} \\
& +C_{\delta} T M^{2}\|v\|_{H^{1}\left(H^{1}\left(\Omega_{F}\right)\right) \cap C^{0}\left(H^{2}\left(\Omega_{F}\right)\right)}^{2} .
\end{aligned}
$$


For the third integral in the right-hand side of (3.16), we use 2.27) to deduce for any $\delta>0$

$$
\begin{aligned}
&\left|\int_{0}^{t} \int_{\Omega_{F}} q\left(\partial_{t} \nabla v\right): \partial_{t} \operatorname{Cof}(\nabla \widehat{X}) d y d s\right| \\
& \leqslant C_{\delta} \int_{0}^{T}\|q\|_{L^{6}\left(\Omega_{F}\right)}^{2}\left\|\partial_{t} \operatorname{Cof}(\nabla \widehat{X})\right\|_{L^{6}\left(\Omega_{F}\right)}^{2} d t+\delta \int_{0}^{T} \int_{\Omega_{F}}\left|\partial_{t} \nabla v\right|^{2} d y d t \\
& \leqslant C_{\delta} T M^{2}\|q\|_{C^{0}\left(H^{1}\left(\Omega_{F}\right)\right)}^{2}+\delta\|v\|_{H^{1}\left(H^{1}\left(\Omega_{F}\right)\right)}^{2} .
\end{aligned}
$$

For the last integral in the right-hand side of 3.16 , we use 2.28 to obtain the following estimate

$$
\begin{aligned}
& \left|\int_{0}^{t} \int_{\Omega_{F}} q \nabla v: \partial_{t t} \operatorname{Cof}(\nabla \widehat{X}) d y d s\right| \\
& \leqslant C T^{1 / 2}\|q\|_{C^{0}\left(L^{6}\left(\Omega_{F}\right)\right)}\|\nabla v\|_{C^{0}\left(L^{6}\left(\Omega_{F}\right)\right)}\left\|\partial_{t t} \operatorname{Cof}(\nabla \widehat{X})\right\|_{L^{2}\left(L^{2}\left(\Omega_{F}\right)\right)} \\
& \leqslant \delta\|q\|_{C^{0}\left(H^{1}\left(\Omega_{F}\right)\right)}^{2}+C_{\delta} T M^{2}\|\nabla v\|_{C^{0}\left(H^{1}\left(\Omega_{F}\right)\right)}^{2} .
\end{aligned}
$$

Gathering the last inequality with 3.19, 3.20 and 3.10, equality 3.16 becomes

$$
\begin{array}{r}
\left|\int_{0}^{t} \int_{\Omega_{F}} \partial_{t}[q \operatorname{Cof}(\nabla \widehat{X})]: \partial_{t} \nabla v d y d s\right| \leqslant C_{\delta}\left(1+\left\|v_{0}\right\|_{H^{2}\left(\Omega_{F}\right)}^{2}\right)\left\|V_{0}\right\|_{H^{2}\left(\Omega_{F}\right)}^{2}+C\left\|G_{0}\right\|_{L^{2}\left(\Omega_{F}\right)}^{2} \\
+\left(3 \delta+C_{\delta} T M^{2}\right)\|q\|_{C^{0}\left(H^{1}\left(\Omega_{F}\right)\right)}^{2} \\
+\left(\delta+C \delta T M^{2}\right)\|v\|_{H^{1}\left(H^{1}\left(\Omega_{F}\right) \cap C^{0}\left(H^{2}\left(\Omega_{F}\right)\right)\right.}^{2} .
\end{array}
$$

Coming back to (3.13, this last inequality, 3.14 and 3.15 imply that

$$
\begin{aligned}
\int_{0}^{t} \int_{\Omega_{F}} \partial_{t}\left\{\left[(\nabla v) \operatorname{Cof}(\nabla \widehat{X})^{*}+\operatorname{Cof}(\nabla \widehat{X})(\nabla v)^{*}-q I_{3}\right] \operatorname{Cof}(\nabla \widehat{X})\right\}: \partial_{t} \nabla v d y d s \\
\geqslant \int_{0}^{t} \int_{\Omega_{F}}\left|\varepsilon\left(\partial_{t} v\right)\right|^{2} d y d s-\left(2 \delta+C_{\delta} T^{1 / 2} M\right)\|v\|_{H^{1}\left(H^{1}\left(\Omega_{F}\right)\right) \cap C^{0}\left(H^{2}\left(\Omega_{F}\right)\right)}^{2} \\
\quad-C_{\delta}\left(1+\left\|v_{0}\right\|_{H^{2}\left(\Omega_{F}\right)}^{2}\right)\left\|V_{0}\right\|_{H^{2}\left(\Omega_{F}\right)}^{2}-C\left\|G_{0}\right\|_{L^{2}\left(\Omega_{F}\right)}^{2}-\left(3 \delta+C_{\delta} T M^{2}\right)\|q\|_{C^{0}\left(H^{1}\left(\Omega_{F}\right)\right)}^{2} .
\end{aligned}
$$

At last, using this inequality, Korn's inequality, $\sqrt{3.9}$ and $\sqrt{3.11}$ in the identity $(3.12)$ integrated in time, we get

$$
\begin{gathered}
\left\|\partial_{t} v\right\|_{C^{0}\left(L^{2}\left(\Omega_{F}\right)\right)}^{2}+\left\|\partial_{t} v\right\|_{L^{2}\left(H^{1}\left(\Omega_{F}\right)\right)}^{2}+\left\|\partial_{t t} \xi\right\|_{C^{0}\left(L^{2}\left(\Omega_{S}\right)\right)}^{2}+\left\|\partial_{t} \xi\right\|_{C^{0}\left(H^{1}\left(\Omega_{S}\right)\right)}^{2} \\
\leqslant C_{\delta}\left(\left(1+\left\|v_{0}\right\|_{H^{2}\left(\Omega_{F}\right)}^{2}\right)\left\|V_{0}\right\|_{H^{2}\left(\Omega_{F}\right)}^{2}+\left\|\Xi_{1}\right\|_{H^{1}\left(\Omega_{S}\right)}^{2}\right)+\left(3 \delta+C M^{2}\right)\|q\|_{C^{0}\left(H^{1}\left(\Omega_{F}\right)\right)}^{2} \\
+\left(2 \delta+C_{\delta} T^{1 / 2} M\right)\|v\|_{H^{1}\left(H^{1}\left(\Omega_{F}\right)\right) \cap C^{0}\left(H^{2}\left(\Omega_{F}\right)\right)}^{2} \\
+C\left(\left\|G_{0}\right\|_{L^{2}\left(\Omega_{F}\right)}^{2}+\|G\|_{H^{1}\left(L^{2}\left(\Omega_{F}\right)\right)}^{2}\right) .
\end{gathered}
$$

\subsection{Elliptic estimates}

In this section, we consider standard elliptic estimates to bound the $H^{2}$ norm in space of the solution. According to 1.14-1.22, the following elliptic problems hold:

$$
\begin{cases}-\operatorname{div} \Sigma(\xi)=-\partial_{t t} \xi & \text { in }(0, T) \times \Omega_{S} \\ \xi(t, \cdot)=\int_{0}^{t} v(s, \cdot) d s & \text { on }(0, T) \times \partial \Omega_{S}\end{cases}
$$

and

$$
\begin{cases}-\operatorname{div} \mathbb{T}(v, q)=-\partial_{t} v+\operatorname{div} R+G & \text { in }(0, T) \times \Omega_{F}, \\ \operatorname{div} v=-\nabla v:\left[\operatorname{Cof}(\nabla \widehat{X})-I_{3}\right] & \text { in }(0, T) \times \Omega_{F}, \\ \mathbb{T}(v, q) n=\Sigma(\xi) n-R n & \text { on }(0, T) \times \partial \Omega_{S}, \\ v=0 & \text { on }(0, T) \times \partial \Omega,\end{cases}
$$


where

$$
R=(\nabla v)\left(\operatorname{Cof}(\nabla \widehat{X})^{*} \operatorname{Cof}(\nabla \widehat{X})-I_{3}\right)+\left[\operatorname{Cof}(\nabla \widehat{X})(\nabla v)^{*} \operatorname{Cof}(\nabla \widehat{X})-(\nabla v)^{*}\right]-q\left[\operatorname{Cof}(\nabla \widehat{X})-I_{3}\right] .
$$

According to Proposition 2.1 $v$ belongs to $C^{0}\left(H^{2}\left(\Omega_{F}\right)\right)$ and $\partial_{t t} \xi$ belongs to $C^{0}\left(L^{2}\left(\Omega_{S}\right)\right)$. Using the elliptic regularity for 3.22 , this implies an estimate of $\xi$ in $C^{0}\left(H^{2}\left(\Omega_{S}\right)\right)$ :

$$
\|\xi\|_{C^{0}\left(H^{2}\left(\Omega_{S}\right)\right)} \leqslant C\left(\left\|\partial_{t t} \xi\right\|_{C^{0}\left(L^{2}\left(\Omega_{S}\right)\right)}+T\|v\|_{C^{0}\left(H^{2}\left(\Omega_{F}\right)\right)}\right) .
$$

Now, using 2.19, 2.20, 2.24 and the embedding $H^{1}\left(\Omega_{F}\right) \subset L^{6}\left(\Omega_{F}\right)$, we have

$$
\|R\|_{C^{0}\left(H^{1}\left(\Omega_{F}\right)\right)} \leqslant C T^{1 / 2} M\left(\|v\|_{C^{0}\left(H^{2}\left(\Omega_{F}\right)\right)}+\|q\|_{C^{0}\left(H^{1}\left(\Omega_{F}\right)\right)}\right),
$$

and

$$
\left\|\nabla v:\left[\operatorname{Cof}(\nabla \widehat{X})-I_{3}\right]\right\|_{C^{0}\left(H^{1}\left(\Omega_{F}\right)\right)} \leqslant C T^{1 / 2} M\|v\|_{C^{0}\left(H^{2}\left(\Omega_{F}\right)\right)} .
$$

Using the elliptic regularity for 3.23 and taking into account (3.26) and (3.27), we deduce

$$
\begin{aligned}
& \|v\|_{C^{0}\left(H^{2}\left(\Omega_{F}\right)\right)}+\|q\|_{C^{0}\left(H^{1}\left(\Omega_{F}\right)\right)} \\
& \qquad C\left(\|G\|_{C^{0}\left(L^{2}\left(\Omega_{F}\right)\right)}+\left\|\partial_{t} v\right\|_{C^{0}\left(L^{2}\left(\Omega_{F}\right)\right)}+\|\Sigma(\xi)\|_{C^{0}\left(H^{1}\left(\Omega_{S}\right)\right)}\right) \\
& \quad+C T^{1 / 2} M\left(\|v\|_{C^{0}\left(H^{2}\left(\Omega_{F}\right)\right)}+\|q\|_{C^{0}\left(H^{1}\left(\Omega_{F}\right)\right)}\right) .
\end{aligned}
$$

Taking

$$
T \leqslant \frac{\eta}{M^{2}}
$$

with $\eta$ small enough, we finally obtain

$$
\begin{aligned}
& \|v\|_{C^{0}\left(H^{2}\left(\Omega_{F}\right)\right)}+\|q\|_{C^{0}\left(H^{1}\left(\Omega_{F}\right)\right)} \\
& \leqslant C\left(\|G\|_{C^{0}\left(L^{2}\left(\Omega_{F}\right)\right)}+\left\|\partial_{t} v\right\|_{C^{0}\left(L^{2}\left(\Omega_{F}\right)\right)}+\|\Sigma(\xi)\|_{C^{0}\left(H^{1}\left(\Omega_{S}\right)\right)}\right) .
\end{aligned}
$$

Combining 3.25 and 3.30 and taking $T \leqslant \eta$ with $\eta$ small enough, we find

$$
\begin{aligned}
\|v\|_{C^{0}\left(H^{2}\left(\Omega_{F}\right)\right)}+\|q\|_{C^{0}\left(H^{1}\left(\Omega_{F}\right)\right)}+ & \|\xi\|_{C^{0}\left(H^{2}\left(\Omega_{S}\right)\right)} \\
& \leqslant C\left(\|G\|_{C^{0}\left(L^{2}\left(\Omega_{F}\right)\right)}+\left\|\partial_{t t} \xi\right\|_{C^{0}\left(L^{2}\left(\Omega_{S}\right)\right)}+\left\|\partial_{t} v\right\|_{C^{0}\left(L^{2}\left(\Omega_{F}\right)\right)}\right) .
\end{aligned}
$$

Then we gather the above estimate and (3.21), and take $\delta$ small enough and then $\eta$ small enough in 3.29. This gives

$$
\begin{aligned}
\|v\|_{C^{1}\left(L^{2}\left(\Omega_{F}\right)\right)}+ & \|v\|_{H^{1}\left(H^{1}\left(\Omega_{F}\right)\right)}+\|v\|_{C^{0}\left(H^{2}\left(\Omega_{F}\right)\right)}+\|q\|_{C^{0}\left(H^{1}\left(\Omega_{F}\right)\right)} \\
& +\|\xi\|_{C^{2}\left(L^{2}\left(\Omega_{S}\right)\right)}+\|\xi\|_{C^{1}\left(H^{1}\left(\Omega_{S}\right)\right)}+\|\xi\|_{C^{0}\left(H^{2}\left(\Omega_{S}\right)\right)} \\
\leqslant C\left(\left(1+\left\|v_{0}\right\|_{H^{2}\left(\Omega_{F}\right)}\right)\left\|V_{0}\right\|_{H^{2}\left(\Omega_{F}\right)}+\left\|\Xi_{1}\right\|_{H^{1}\left(\Omega_{S}\right)}+\|G\|_{H^{1}\left(L^{2}\left(\Omega_{F}\right)\right) \cap C^{0}\left(L^{2}\left(\Omega_{F}\right)\right)}\right) &
\end{aligned}
$$

with a constant $C$ independent of $\left(V_{0}, \Xi_{1}, G\right)$, of $T$ and of $M$.

\subsection{Study of a linear Stokes system}

In order to complete the proof of Theorem 1.4 we still have to estimate $(v, q)$ in $L^{2}\left(H^{5 / 2+1 / 8}\left(\Omega_{F}\right)\right) \times$ $L^{2}\left(H^{3 / 2+1 / 8}\left(\Omega_{F}\right)\right)$ and $\xi$ in $C^{1}\left(H^{1+1 / 8}\left(\Omega_{S}\right)\right) \cap C^{0}\left(H^{2+1 / 8}\left(\Omega_{S}\right)\right)$. This is done in Subsection 3.5 These additional estimates with more regular spatial norms will be achieved by using some specific results satisfied by the solution of the Stokes system. These results are presented in this subsection and involve some duality arguments. 
Our aim is to study the following Stokes type system

$$
\begin{cases}\partial_{t} w-\operatorname{div} \mathbb{T}_{\widehat{X}}\left(w, \pi_{w}\right)=f_{1} & \text { in }(0, T) \times \Omega_{F}, \\ \nabla w: \operatorname{Cof}(\nabla \widehat{X})=f_{2} & \text { in }(0, T) \times \Omega_{F}, \\ \mathbb{T}_{\widehat{X}}\left(w, \pi_{w}\right) n=f_{3} & \text { on }(0, T) \times \partial \Omega_{S}, \\ w=f_{4} & \text { on }(0, T) \times \partial \Omega, \\ w(0, \cdot)=w_{0} & \text { in } \Omega_{F},\end{cases}
$$

where $\mathbb{T}_{\widehat{X}}$ is defined by $(1.6)$.

In order to study this system, we introduce the adjoint system

$$
\begin{cases}-\partial_{t} \varphi-\operatorname{div} \mathbb{T}_{\widehat{X}}\left(\varphi, \pi_{\varphi}\right)=f & \text { in }(0, T) \times \Omega_{F}, \\ \nabla \varphi: \operatorname{Cof}(\nabla \widehat{X})=0 & \text { in }(0, T) \times \Omega_{F}, \\ \mathbb{T}_{\widehat{X}}\left(\varphi, \pi_{\varphi}\right) n=0 & \text { on }(0, T) \times \partial \Omega_{S}, \\ \varphi=0 & \text { on }(0, T) \times \partial \Omega, \\ \varphi(T, \cdot)=0 & \text { in } \Omega_{F} .\end{cases}
$$

We also need to lift the "divergence condition", that is to consider the following stationary system

$$
\begin{cases}-\operatorname{div} \mathbb{T}_{\widehat{X}}\left(W, \pi_{W}\right)=0 & \text { in }(0, T) \times \Omega_{F}, \\ \nabla W: \operatorname{Cof}(\nabla \widehat{X})=f_{2} & \text { in }(0, T) \times \Omega_{F}, \\ \mathbb{T}_{\widehat{X}}\left(W, \pi_{W}\right) n=0 & \text { on }(0, T) \times \partial \Omega_{S}, \\ W=0 & \text { on }(0, T) \times \partial \Omega\end{cases}
$$

Lemma 3.1. Let $\widehat{X} \in B_{M}$. There exist $\alpha>0$ and $\eta>0$ such that for any $T$ such that

$$
T^{\alpha} M \leqslant \eta
$$

and for any

$$
\begin{gathered}
w_{0} \in H^{1}\left(\Omega_{F}\right), \quad f_{1} \in L^{2}\left(0, T ; L^{2}\left(\Omega_{F}\right)\right), \quad f_{2} \in H^{1}\left(0, T ; H^{-1}\left(\Omega_{F}\right)\right) \cap L^{2}\left(0, T ; H^{1}\left(\Omega_{F}\right)\right), \\
f_{3} \in L^{2}\left(0, T ; H^{1 / 2}\left(\partial \Omega_{S}\right)\right) \cap H^{1 / 4}\left(0, T ; L^{2}\left(\partial \Omega_{S}\right)\right), \\
f_{4} \in L^{2}\left(0, T ; H^{3 / 2}(\partial \Omega)\right) \cap H^{3 / 4}\left(0, T ; L^{2}(\partial \Omega)\right),
\end{gathered}
$$

system (3.33) admits a unique strong solution

$$
w \in L^{2}\left(0, T ; H^{2}\left(\Omega_{F}\right)\right) \cap H^{1}\left(0, T ; L^{2}\left(\Omega_{F}\right)\right) .
$$

Moreover, we have the estimates

$$
\begin{aligned}
& \|w\|_{L^{2}\left(0, T ; H^{2}\left(\Omega_{F}\right)\right) \cap H^{1}\left(0, T ; L^{2}\left(\Omega_{F}\right)\right)} \leqslant C\left(\left\|f_{1}\right\|_{L^{2}\left(0, T ; L^{2}\left(\Omega_{F}\right)\right)}+\left\|f_{2}\right\|_{H^{1}\left(0, T ; H^{-1}\left(\Omega_{F}\right)\right) \cap L^{2}\left(0, T ; H^{1}\left(\Omega_{F}\right)\right)}\right. \\
& \left.\quad+\left\|f_{3}\right\|_{L^{2}\left(0, T ; H^{1 / 2}\left(\partial \Omega_{S}\right)\right) \cap H^{1 / 4}\left(0, T ; L^{2}\left(\partial \Omega_{S}\right)\right)}+\left\|f_{4}\right\|_{L^{2}\left(0, T ; H^{3 / 2}(\partial \Omega)\right) \cap H^{3 / 4}\left(0, T ; L^{2}(\partial \Omega)\right)}+\left\|w_{0}\right\|_{H^{1}\left(\Omega_{F}\right)}\right) .
\end{aligned}
$$

Similarly, if $f \in L^{2}\left(0, T ; L^{2}\left(\Omega_{F}\right)\right)$, there exists a strong solution

$$
\varphi \in L^{2}\left(0, T ; H^{2}\left(\Omega_{F}\right)\right) \cap H^{1}\left(0, T ; L^{2}\left(\Omega_{F}\right)\right), \pi_{\varphi} \in L^{2}\left(0, T ; H^{1}\left(\Omega_{F}\right)\right)
$$

of 3.34 and we have the estimate

$$
\|\varphi\|_{L^{2}\left(0, T ; H^{2}\left(\Omega_{F}\right)\right) \cap H^{1}\left(0, T ; L^{2}\left(\Omega_{F}\right)\right)}+\left\|\pi_{\varphi}\right\|_{L^{2}\left(0, T ; H^{1}\left(\Omega_{F}\right)\right)} \leqslant C\|f\|_{L^{2}\left(0, T ; L^{2}\left(\Omega_{F}\right)\right)} .
$$


The proof of the above result is completely similar to Proposition 2.1. with a fixed point argument and by using the estimates on $\widehat{X}$ presented in Subsection 2.1 We thus skip the proof.

The fact that (3.34) is an adjoint system of $(3.33)$ can be seen by multiplying (3.33) by $\varphi,(3.34)$ by $w$ and by using integrations by parts. By this way, we get

$$
\begin{aligned}
\int_{0}^{T} \int_{\Omega_{F}} w \cdot f d x d t=\int_{\Omega_{F}} w_{0} \cdot \varphi(0, \cdot) d x & +\int_{0}^{T} \int_{\Omega_{F}} f_{1} \cdot \varphi d x d t-\int_{0}^{T} \int_{\Omega_{F}} f_{2} \pi_{\varphi} d x d t \\
& +\int_{0}^{T} \int_{\partial \Omega_{S}} f_{3} \cdot \varphi d x d t-\int_{0}^{T} \int_{\partial \Omega} f_{4} \cdot \mathbb{T}_{\widehat{X}}\left(\varphi, \pi_{\varphi}\right) n d x d t .
\end{aligned}
$$

This yields the following result on (3.34):

Lemma 3.2. Let $\widehat{X} \in B_{M}$. Assume (3.36) and $f \in H^{-1 / 3}\left(0, T ; L^{2}\left(\Omega_{F}\right)\right)$. Then there exists a weak solution

$$
\varphi \in L^{2}\left(0, T ; H^{4 / 3}\left(\Omega_{F}\right)\right) \cap H^{2 / 3}\left(0, T ; L^{2}\left(\Omega_{F}\right)\right)
$$

of 3.34. Moreover, we have the estimates

$$
\|\varphi\|_{L^{2}\left(0, T ; H^{4 / 3}\left(\Omega_{F}\right)\right) \cap H^{2 / 3}\left(0, T ; L^{2}\left(\Omega_{F}\right)\right)} \leqslant C\|f\|_{H^{-1 / 3}\left(0, T ; L^{2}\left(\Omega_{F}\right)\right)}
$$

and

$$
T^{-2 / 3}\|\varphi\|_{L^{2}\left((0, T) \times \Omega_{F}\right)}+T^{-1 / 6}\|\varphi\|_{C^{0}\left([0, T] ; L^{2}\left(\Omega_{F}\right)\right)} \leqslant C\|f\|_{H^{-1 / 3}\left(0, T ; L^{2}\left(\Omega_{F}\right)\right)} .
$$

Proof. Lemma 3.1 and formula 3.39 with

$$
f_{2}=0, \quad f_{3}=0, \quad f_{4}=0, \quad w_{0}=0 .
$$

show that there exists a weak solution $\varphi \in L^{2}\left((0, T) \times \Omega_{F}\right)$ of 3.34 for any $f \in\left(H_{L}^{1}\right)^{\prime}\left(0, T ; L^{2}\left(\Omega_{F}\right)\right)$ defined by

$$
\int_{0}^{T} \int_{\Omega_{F}} \varphi \cdot f_{1} d x d t=\langle f, w\rangle_{\left(H_{L}^{1}\right)^{\prime}\left(0, T ; L^{2}\left(\Omega_{F}\right)\right), H_{L}^{1}\left(0, T ; L^{2}\left(\Omega_{F}\right)\right)} .
$$

We recall that for $s \in(1 / 2,3 / 2), H_{L}^{s}(0, T ; \mathcal{X})$ is the subspace of of functions $f \in H^{s}(0, T ; \mathcal{X})$ such that $f(0)=0$. We also use $H_{R}^{s}(0, T ; \mathcal{X})$ in the case where $f(T)=0$ instead of $f(0)=0$. From (3.37), we deduce that

$$
\|\varphi\|_{L^{2}\left((0, T) \times \Omega_{F}\right)} \leqslant C\|f\|_{\left(H_{L}^{1}\right)^{\prime}\left(0, T ; L^{2}\left(\Omega_{F}\right)\right)} .
$$

By interpolation, this yields that if $f \in H^{-1 / 3}\left(0, T ; L^{2}\left(\Omega_{F}\right)\right)$, then $\varphi$ satisfies 3.40 .

In order to show 3.41 and 3.42 with a constant $C$ independent of $T$, we need to use the change of variables A.5 of the appendix and we define the linear map

$$
\Lambda_{1}: L^{2}\left((0,1) \times \Omega_{F}\right) \rightarrow L^{2}\left(0,1 ; H^{2}\left(\Omega_{F}\right)\right) \cap H^{1}\left(0,1 ; L^{2}\left(\Omega_{F}\right)\right)
$$

defined by $\Lambda_{1}\left(f^{*}\right)=\varphi^{*}$, where $f^{*}$ and $\varphi^{*}$ are the transformations of $f$ and $\varphi$ through A.5. From (A.6), A.7 and 3.38, we have

$$
\left\|\Lambda_{1}\right\|_{\mathcal{L}\left(L^{2}\left((0,1) \times \Omega_{F}\right), L^{2}\left(0,1 ; H^{2}\left(\Omega_{F}\right)\right)\right)} \leqslant C \quad \text { and } \quad\left\|\Lambda_{1}\right\|_{\mathcal{L}\left(L^{2}\left((0,1) \times \Omega_{F}\right), H_{R}^{1}\left(0,1 ; L^{2}\left(\Omega_{F}\right)\right)\right)} \leqslant C T .
$$

Using (A.6), A.7) and (3.37) and the definition of a weak solution of (3.34), we also deduce

$$
\left\|\Lambda_{1}\right\|_{\mathcal{L}\left(\left(H_{L}^{1}\right)^{\prime}\left(0,1 ; L^{2}\left(\Omega_{F}\right)\right), L^{2}\left((0,1) \times \Omega_{F}\right)\right.} \leqslant C T .
$$

Using an interpolation argument and (3.46), 3.47), we deduce

$$
\left\{\begin{array}{l}
\left\|\Lambda_{1}\right\|_{\mathcal{L}\left(H^{-1 / 3}\left(0,1 ; L^{2}\left(\Omega_{F}\right)\right), H_{R}^{2 / 3}\left(0,1 ; L^{2}\left(\Omega_{F}\right)\right)\right.} \leqslant C T, \\
\left\|\Lambda_{1}\right\|_{\mathcal{L}\left(H^{-1 / 3}\left(0,1 ; L^{2}\left(\Omega_{F}\right)\right), L^{2}\left(0,1 ; H^{4 / 3}\left(\Omega_{F}\right)\right)\right.} \leqslant C T^{1 / 3} .
\end{array}\right.
$$

Furthermore, from A.6, Lemma A.1 and Corollary A.4 we have

$$
\begin{gathered}
\left\|\varphi^{*}\right\|_{H_{R}^{2 / 3}\left(0,1 ; L^{2}\left(\Omega_{F}\right)\right)}=T^{1 / 6}\|\varphi\|_{H_{R}^{2 / 3}\left(0, T ; L^{2}\left(\Omega_{F}\right)\right)}, \\
\left\|\varphi^{*}\right\|_{L^{2}\left(0,1 ; H^{4 / 3}\left(\Omega_{F}\right)\right)}=T^{-1 / 2}\|\varphi\|_{L^{2}\left(0, T ; H^{4 / 3}\left(\Omega_{F}\right)\right)}, \\
\left\|f^{*}\right\|_{H^{-1 / 3}\left(0,1 ; L^{2}\left(\Omega_{F}\right)\right)} \leqslant T^{-5 / 6}\|f\|_{H^{-1 / 3}\left(0, T ; L^{2}\left(\Omega_{F}\right)\right)}
\end{gathered}
$$


From the above relation and 3.48, we obtain 3.41. Then, 3.42 comes from 3.41 and from the two relations

$$
\|\varphi\|_{L^{2}\left(0, T ; L^{2}\left(\Omega_{F}\right)\right)} \leqslant C T^{2 / 3}\|\varphi\|_{H_{L}^{2 / 3}\left(0, T ; L^{2}\left(\Omega_{F}\right)\right)}, \quad\|\varphi\|_{C^{0}\left([0, T] ; L^{2}\left(\Omega_{F}\right)\right)} \leqslant C T^{1 / 6}\|\varphi\|_{H_{L}^{2 / 3}\left(0, T ; L^{2}\left(\Omega_{F}\right)\right)} .
$$

Using again (3.39), we deduce from Lemma 3.2 a result for the system 3.33 in the case

$$
f_{2}=0, \quad f_{4}=0 .
$$

Proposition 3.3. Let $\widehat{X} \in B_{M}$. Assume 3.49, 3.36 and

$$
f_{1} \in L^{2}\left(0, T ; L^{2}\left(\Omega_{F}\right)\right), \quad f_{3} \in L^{2}\left(0, T ; L^{2}\left(\partial \Omega_{S}\right)\right), \quad w_{0} \in L^{2}\left(\Omega_{F}\right) .
$$

Then there exists a weak solution

$$
w \in H^{1 / 3}\left(0, T ; L^{2}\left(\Omega_{F}\right)\right)
$$

of 3.33. Moreover, we have the estimates

$$
\left.\|w\|_{H^{1 / 3}\left(0, T ; L^{2}\left(\Omega_{F}\right)\right)} \leqslant C\left(T^{2 / 3}\left\|f_{1}\right\|_{L^{2}\left(0, T ; L^{2}\left(\Omega_{F}\right)\right)}+\left\|f_{3}\right\|_{L^{2}\left(0, T ; L^{2}\left(\partial \Omega_{S}\right)\right)}+T^{1 / 6}\left\|w_{0}\right\|_{L^{2}\left(\Omega_{F}\right)}\right)\right) .
$$

Proof. The solution of 3.33 is defined by duality with system 3.34$)$ : for any $f \in H^{-1 / 3}\left(0, T ; L^{2}\left(\Omega_{F}\right)\right)$, we consider the solution $\varphi$ of (3.34) given in Lemma 3.2 and $w$ is defined by

$$
\begin{aligned}
\langle w, f\rangle_{H^{1 / 3}\left(0, T ; L^{2}\left(\Omega_{F}\right)\right), H^{-1 / 3}\left(0, T ; L^{2}\left(\Omega_{F}\right)\right)}:=\int_{0}^{T} \int_{\Omega_{F}} f_{1} \cdot \varphi d x d t & +\int_{0}^{T} \int_{\partial \Omega_{S}} f_{3} \cdot \varphi d \gamma d t \\
& +\int_{\Omega_{F}} w_{0} \cdot \varphi(0, \cdot) d x .
\end{aligned}
$$

We use 3.41 and 3.42 to deduce 3.50.

Let us now consider the stationary system (3.35).

Lemma 3.4. Let $\widehat{X} \in B_{M}$. Assume 3.36 and

$$
f_{2} \in H^{1}\left(0, T ; L^{3 / 2}\left(\Omega_{F}\right)\right) \cap L^{2}\left(0, T ; H^{1}\left(\Omega_{F}\right)\right) .
$$

Then there exists

$$
W \in H^{1}\left(0, T ; L^{2}\left(\Omega_{F}\right)\right) \cap L^{2}\left(0, T ; H^{2}\left(\Omega_{F}\right)\right), \quad \pi_{W} \in L^{2}\left(0, T ; H^{1}\left(\Omega_{F}\right)\right)
$$

with

$$
\begin{aligned}
\|W\|_{H^{1}\left(0, T ; L^{2}\left(\Omega_{F}\right)\right) \cap L^{2}\left(0, T ; H^{2}\left(\Omega_{F}\right)\right)}+\left\|\pi_{W}\right\|_{L^{2}\left(0, T ; H^{1}\left(\Omega_{F}\right)\right)} & \\
& \leqslant C M\left\|f_{2}\right\|_{L^{2}\left(0, T ; H^{1}\left(\Omega_{F}\right)\right)}+C\left\|f_{2}\right\|_{H^{1}\left(0, T ; L^{3 / 2}\left(\Omega_{F}\right)\right)} .
\end{aligned}
$$

Proof. We write 3.35 as

$$
\begin{cases}-\operatorname{div} \mathbb{T}\left(W, \pi_{W}\right)=\operatorname{div} \widehat{F} & \text { in }(0, T) \times \Omega_{F}, \\ \operatorname{div} W=\widehat{F}_{2} & \text { in }(0, T) \times \Omega_{F}, \\ \mathbb{T}\left(W, \pi_{W}\right) n=-\widehat{F} n & \text { on }(0, T) \times \partial \Omega_{S}, \\ W=0 & \text { on }(0, T) \times \partial \Omega,\end{cases}
$$

with

$$
\begin{gathered}
\widehat{F}=\mathbb{T}_{\widehat{X}}\left(W, \pi_{W}\right)-\mathbb{T}\left(W, \pi_{W}\right), \\
\widehat{F}_{2}=f_{2}+\nabla W:\left(I_{3}-\operatorname{Cof}(\nabla \widehat{X})\right) .
\end{gathered}
$$


We recall that $\mathbb{T}_{\widehat{X}}$ is given by 1.6 . Assume that $\left(W, \pi_{W}\right) \in L^{2}\left(0, T ; H^{2}\left(\Omega_{F}\right)\right) \times L^{2}\left(0, T ; H^{1}\left(\Omega_{F}\right)\right)$ and let us consider $\widehat{F}$ and $\widehat{F}_{2}$ defined by the above formulas. Using Section 2.1 we can show that

$$
\begin{aligned}
& \|\operatorname{div} \widehat{F}\|_{L^{2}\left((0, T) \times \Omega_{F}\right)}+\left\|\widehat{F}_{2}\right\|_{L^{2}\left((0, T) ; H^{1}\left(\Omega_{F}\right)\right)}+\|\widehat{F} n\|_{L^{2}\left(0, T ; H^{1 / 2}\left(\partial \Omega_{S}\right)\right)} \\
& \quad \leqslant C\left(\left\|f_{2}\right\|_{L^{2}\left(0, T ; H^{1}\left(\Omega_{F}\right)\right)}+T^{\alpha} M\left[\|W\|_{L^{2}\left(0, T ; H^{2}\left(\Omega_{F}\right)\right)}+\left\|\pi_{W}\right\|_{L^{2}\left(0, T ; H^{1}\left(\Omega_{F}\right)\right)}\right]\right) .
\end{aligned}
$$

Using the standard elliptic estimates for the Stokes system and the above estimate, we can prove in a similar way as Proposition 2.1 (with a fixed point argument) the existence and uniqueness of the solution $\left(W, \pi_{W}\right) \in L^{2}\left(0, T ; H^{2}\left(\Omega_{F}\right)\right) \times L^{2}\left(0, T ; H^{1}\left(\Omega_{F}\right)\right)$ of 3.35 (by assuming $(3.36)$. Moreover, we have the estimate

$$
\|W\|_{L^{2}\left(0, T ; H^{2}\left(\Omega_{F}\right)\right)}+\left\|\pi_{W}\right\|_{L^{2}\left(0, T ; H^{1}\left(\Omega_{F}\right)\right)} \leqslant C\left\|f_{2}\right\|_{L^{2}\left(0, T ; H^{1}\left(\Omega_{F}\right)\right)} .
$$

To obtain an estimate of $W$ in $H^{1}\left(0, T ; L^{2}\left(\Omega_{F}\right)\right)$, we differentiate 3.35 with respect to time:

$$
\begin{cases}-\operatorname{div} \mathbb{T}_{\widehat{X}}\left(\partial_{t} W, \partial_{t} \pi_{W}\right)=\operatorname{div} \breve{F} & \text { in }(0, T) \times \Omega_{F}, \\ \nabla \partial_{t} W: \operatorname{Cof}(\nabla \widehat{X})=\breve{F}_{2} & \text { in }(0, T) \times \Omega_{F}, \\ \mathbb{T}_{\widehat{X}}\left(\partial_{t} W, \partial_{t} \pi_{W}\right) n=-\breve{F} n & \text { on }(0, T) \times \partial \Omega_{S}, \\ \partial_{t} W=0 & \text { on }(0, T) \times \partial \Omega,\end{cases}
$$

with

$$
\begin{gathered}
\breve{F}=\left\{\left[(\nabla W) \partial_{t} \operatorname{Cof}(\nabla \widehat{X})^{*}+\partial_{t} \operatorname{Cof}(\nabla \widehat{X})(\nabla W)^{*}\right] \operatorname{Cof}(\nabla \widehat{X})\right\} \\
+\left\{\left[(\nabla W) \operatorname{Cof}(\nabla \widehat{X})^{*}+\operatorname{Cof}(\nabla \widehat{X})(\nabla W)^{*}-\pi_{W} I_{3}\right] \partial_{t} \operatorname{Cof}(\nabla \widehat{X})\right\} \\
\breve{F}_{2}=\partial_{t} f_{2}+\nabla W:\left(\partial_{t} \operatorname{Cof}(\nabla \widehat{X})\right) .
\end{gathered}
$$

System (3.58) is satisfied in a weak sense: from the first part of the proof, we know that if $(3.36)$ holds, for any $f \in L^{2}\left(0, T ; L^{2}\left(\Omega_{F}\right)\right)$, there exists a unique solution $\varphi \in L^{2}\left(0, T ; H^{2}\left(\Omega_{F}\right)\right), \pi_{\varphi} \in L^{2}\left(0, T ; H^{1}\left(\Omega_{F}\right)\right)$ of

$$
\begin{cases}-\operatorname{div} \mathbb{T}_{\widehat{X}}\left(\varphi, \pi_{\varphi}\right)=f & \text { in }(0, T) \times \Omega_{F}, \\ \nabla \varphi: \operatorname{Cof}(\nabla \widehat{X})=0 & \text { in }(0, T) \times \Omega_{F}, \\ \mathbb{T}_{\widehat{X}}\left(\varphi, \pi_{\varphi}\right) n=0 & \text { on }(0, T) \times \partial \Omega_{S}, \\ \varphi=0 & \text { on }(0, T) \times \partial \Omega .\end{cases}
$$

Then, for general $\breve{F}, \breve{F}_{2} \in L^{2}\left(0, T ; L^{6 / 5}\left(\Omega_{F}\right)\right)$, we can define the solution of 3.58 as the function

$$
\partial_{t} W \in L^{2}\left(0, T ; L^{2}\left(\Omega_{F}\right)\right)
$$

such that

$$
\int_{0}^{T} \int_{\Omega_{F}} \partial_{t} W \cdot f d x d t:=\int_{0}^{T} \int_{\Omega_{F}}\left(\breve{F}: \nabla \varphi+\breve{F}_{2} \pi_{\varphi}\right) d x d t .
$$

Moreover, thanks to 3.38 we have

$$
\left\|\partial_{t} W\right\|_{L^{2}\left(0, T ; L^{2}\left(\Omega_{F}\right)\right)} \leqslant C\left(\|\breve{F}\|_{L^{2}\left(0, T ; L^{6 / 5}\left(\Omega_{F}\right)\right)}+\left\|\breve{F}_{2}\right\|_{L^{2}\left(0, T ; L^{6 / 5}\left(\Omega_{F}\right)\right)}\right)
$$

where $C$ is independent of $T$.

From 2.19, 2.27), 3.59, 3.60, we deduce

$$
\begin{aligned}
\left\|\breve{F}_{2}\right\|_{L^{2}\left(0, T ; L^{6 / 5}\left(\Omega_{F}\right)\right)} & \leqslant\left\|\partial_{t} f_{2}\right\|_{L^{2}\left(0, T ; L^{6 / 5}\left(\Omega_{F}\right)\right)}+\left\|\nabla W:\left(\partial_{t} \operatorname{Cof}(\nabla \widehat{X})\right)\right\|_{L^{2}\left(0, T ; L^{6 / 5}\left(\Omega_{F}\right)\right)} \\
& \leqslant C\left\|\partial_{t} f_{2}\right\|_{L^{2}\left(0, T ; L^{3 / 2}\left(\Omega_{F}\right)\right)}+C M\|\nabla W\|_{L^{2}\left(0, T ; L^{6}\left(\Omega_{F}\right)\right)}
\end{aligned}
$$

and

$$
\|\breve{F}\|_{L^{2}\left(0, T ; L^{6 / 5}\left(\Omega_{F}\right)\right)} \leqslant C M\left(\|\nabla W\|_{L^{2}\left(0, T ; L^{6}\left(\Omega_{F}\right)\right)}+\left\|\pi_{W}\right\|_{L^{2}\left(0, T ; L^{6}\left(\Omega_{F}\right)\right)}\right) .
$$

Combining the above relations, 3.57 and 3.63, we obtain 3.52. 


\subsection{Estimate of the $H^{5 / 2+1 / 8}\left(\Omega_{F}\right)$-norm}

In order to obtain estimates on $(v, q)$ in $L^{2}\left(H^{5 / 2+1 / 8}\left(\Omega_{F}\right)\right) \times L^{2}\left(H^{3 / 2+1 / 8}\left(\Omega_{F}\right)\right)$, we are going to use the ellipticity of the stationary Stokes system 3.23 . To do so, the main difficulty will come from the boundary term $\Sigma(\xi) n$ that we need to estimate in $L^{2}\left(H^{1+1 / 8}\left(\partial \Omega_{S}\right)\right)$. This will be done by applying a hidden regularity result 25] and by proving some bounds on $\xi$ in $H^{2+1 / 8}\left((0, T) \times \partial \Omega_{S}\right)$. Since $\partial_{t} \xi=$ $v$ on $(0, T) \times \partial \Omega_{S}$, we first work on the system satisfied by $\left(\partial_{t} v, \partial_{t} q\right)$ in order to prove that $\partial_{t} v$ in $H^{1 / 8}\left(L^{2}\left(\partial \Omega_{S}\right)\right)$. From Lemma A.9 it is in fact sufficient to estimate $\partial_{t} v$ in $H^{1 / 3}\left(L^{2}\left(\Omega_{F}\right)\right)$. This will be done in the next proposition with the help of Proposition 3.3 and Lemma 3.4 . A key point of the proof will be to estimate the term $\Sigma\left(\partial_{t} \xi\right) n$ that appears as a boundary term in the system of $\left(\partial_{t} v, \partial_{t} q\right)$. At this step again, we will use a hidden regularity result stated in [25].

We will prove the following result:

Proposition 3.5. Assume $\widehat{X} \in B_{M},\left\|v_{0}\right\|_{H^{2}(\Omega)} \leqslant M$ and 3.36. Then the solution of system 1.14(1.22) satisfies

$$
\partial_{t} v \in H^{1 / 3}\left(L^{2}\left(\Omega_{F}\right)\right)
$$

with the estimate

$$
\begin{array}{r}
\left\|\partial_{t} v\right\|_{H^{1 / 3}\left(L^{2}\left(\Omega_{F}\right)\right)} \leqslant C\left(\left(1+\left\|v_{0}\right\|_{H^{2}\left(\Omega_{F}\right)}\right)\left\|V_{0}\right\|_{H^{2}\left(\Omega_{F}\right)}+\left\|\Xi_{1}\right\|_{H^{1}\left(\Omega_{S}\right)}+\|G\|_{H^{1}\left(L^{2}\left(\Omega_{F}\right)\right) \cap C^{0}\left(L^{2}\left(\Omega_{F}\right)\right)}\right) \\
+C T^{1 / 10} M\left(\|v\|_{L^{2}\left(H^{5 / 2+1 / 8}\left(\Omega_{F}\right)\right)}+\|q\|_{L^{2}\left(H^{3 / 2+1 / 8}\left(\Omega_{F}\right)\right)}\right) .
\end{array}
$$

Proof. From system $3.3-(3.9)$, we deduce that $\left(\partial_{t} v, \partial_{t} q\right)$ satisfies 3.33 with

$$
f_{1}:=\partial_{t} G+\operatorname{div} r, \quad f_{2}:=-\nabla v: \partial_{t} \operatorname{Cof}(\nabla \widehat{X}), \quad f_{3}:=-r n+\Sigma\left(\partial_{t} \xi\right) n, \quad f_{4}=0, \quad w_{0}=V_{1}
$$

and

$$
\begin{aligned}
r=\left\{\left[(\nabla v) \partial_{t} \operatorname{Cof}(\nabla \widehat{X})^{*}+\partial_{t} \operatorname{Cof}(\nabla \widehat{X})(\nabla v)^{*}\right] \operatorname{Cof}(\nabla \widehat{X})\right\} \\
+\left\{\left[(\nabla v) \operatorname{Cof}(\nabla \widehat{X})^{*}+\operatorname{Cof}(\nabla \widehat{X})(\nabla v)^{*}-q I_{3}\right] \partial_{t} \operatorname{Cof}(\nabla \widehat{X})\right\} .
\end{aligned}
$$

Let us consider $\left(W, \pi_{W}\right)$ the solution of the stationary system 3.35 associated with $f_{2}$ given in (3.65). We write

$$
\check{v}:=\partial_{t} v-W \quad \text { and } \quad \check{q}:=\partial_{t} q-\pi_{W} .
$$

Then

$$
\begin{cases}\partial_{t} \check{v}-\operatorname{div} \mathbb{T}_{\widehat{X}}(\check{v}, \check{q})=f_{1}-\partial_{t} W & \text { in }(0, T) \times \Omega_{F}, \\ \nabla \check{v}: \operatorname{Cof}(\nabla \widehat{X})=0 & \text { in }(0, T) \times \Omega_{F}, \\ \mathbb{T}_{\widehat{X}}(\check{v}, \check{q}) n=f_{3} & \text { on }(0, T) \times \partial \Omega_{S}, \\ \check{v}=0 & \text { on }(0, T) \times \partial \Omega, \\ \check{v}(0, \cdot)=V_{1}-W(0, \cdot) & \text { in } \Omega_{F} .\end{cases}
$$

We can apply Proposition 3.3 and we deduce

$$
\begin{aligned}
\left\|\partial_{t} v\right\|_{H^{1 / 3}\left(L^{2}\left(\Omega_{F}\right)\right)} \leqslant C\left(T^{2 / 3}\left\|f_{1}-\partial_{t} W\right\|_{L^{2}\left(L^{2}\left(\Omega_{F}\right)\right)}+\left\|f_{3}\right\|_{L^{2}\left((0, T) \times \partial \Omega_{S}\right)}\right. \\
\left.+T^{1 / 6}\left\|V_{1}-W(0, \cdot)\right\|_{L^{2}\left(\Omega_{F}\right)}\right)+\|W\|_{H^{1 / 3}\left(L^{2}\left(\Omega_{F}\right)\right)} .
\end{aligned}
$$

Using Corollary A.3 for $\sigma_{1}=1$ and $s_{1}=1 / 3$, we have, for the last term

$$
\begin{aligned}
\|W\|_{H^{1 / 3}\left(L^{2}\left(\Omega_{F}\right)\right)} & \leqslant\|W-W(0, \cdot)\|_{H^{1 / 3}\left(L^{2}\left(\Omega_{F}\right)\right)}+\|W(0, \cdot)\|_{H^{1 / 3}\left(L^{2}\left(\Omega_{F}\right)\right)} \\
& \leqslant C T^{2 / 3}\|W-W(0, \cdot)\|_{H^{1}\left(L^{2}\left(\Omega_{F}\right)\right)}+C\|W(0, \cdot)\|_{L^{2}\left(\Omega_{F}\right)} \\
& \leqslant C T^{2 / 3}\|W\|_{H^{1}\left(L^{2}\left(\Omega_{F}\right)\right)}+C\|W(0, \cdot)\|_{L^{2}\left(\Omega_{F}\right)} .
\end{aligned}
$$

Thus inequality 3.68 becomes

$$
\begin{aligned}
\left\|\partial_{t} v\right\|_{H^{1 / 3}\left(L^{2}\left(\Omega_{F}\right)\right)} \leqslant C\left(T^{2 / 3}\left\|f_{1}\right\|_{L^{2}\left(L^{2}\left(\Omega_{F}\right)\right)}+T^{2 / 3}\|W\|_{H^{1}\left(L^{2}\left(\Omega_{F}\right)\right)}+\left\|f_{3}\right\|_{L^{2}\left((0, T) \times \partial \Omega_{S}\right)}\right. \\
\left.+T^{1 / 6}\left\|V_{1}\right\|_{L^{2}\left(\Omega_{F}\right)}+\|W(0, \cdot)\|_{L^{2}\left(\Omega_{F}\right)}\right) .
\end{aligned}
$$


Using Lemma 3.4 (see 3.52 ), we have

$$
\|W\|_{H^{1}\left(L^{2}\left(\Omega_{F}\right)\right)} \leqslant C\left(M\left\|f_{2}\right\|_{L^{2}\left(H^{1}\left(\Omega_{F}\right)\right)}+\left\|f_{2}\right\|_{H^{1}\left(L^{3 / 2}\left(\Omega_{F}\right)\right)}\right) .
$$

Let us estimate the two terms in the right-hand side of this last inequality. First, using Lemma A.6. we deduce that

$$
\begin{aligned}
& \left\|\nabla^{2} v\right\|_{L^{2}\left(L^{3}\left(\Omega_{F}\right)\right)} \leqslant C T^{1 / 10}\|v\|_{L^{2}\left(H^{5 / 2+1 / 8}\left(\Omega_{F}\right)\right) \cap C^{0}\left(H^{2}\left(\Omega_{F}\right)\right)}, \\
& \|\nabla q\|_{L^{2}\left(L^{3}\left(\Omega_{F}\right)\right)} \leqslant C T^{1 / 10}\|q\|_{L^{2}\left(H^{3 / 2+1 / 8}\left(\Omega_{F}\right)\right) \cap C^{0}\left(H^{1}\left(\Omega_{F}\right)\right)} .
\end{aligned}
$$

Second, we notice that $\nabla f_{2}$ is a linear combination of

$$
\frac{\partial}{\partial t}[\operatorname{Cof}(\nabla \widehat{X})]_{i_{1}, i_{2}} \frac{\partial^{2} v_{i_{3}}}{\partial y_{i_{4}} \partial y_{i_{5}}}, \quad \frac{\partial^{2}}{\partial t \partial y_{i_{3}}}[\operatorname{Cof}(\nabla \widehat{X})]_{i_{1}, i_{2}} \frac{\partial v_{i_{4}}}{\partial y_{i_{5}}},
$$

with $i_{1}, \ldots, i_{5} \in\{1,2,3\}$ whereas

$$
\partial_{t} f_{2}=-\nabla \partial_{t} v: \partial_{t} \operatorname{Cof}(\nabla \widehat{X})-\nabla v: \partial_{t t} \operatorname{Cof}(\nabla \widehat{X}) .
$$

Using 3.72 with 3.71, 2.27, 2.29, 3.32 and 3.36), we deduce

$$
\begin{aligned}
&\left\|f_{2}\right\|_{L^{2}\left(H^{1}\left(\Omega_{F}\right)\right)} \leqslant C T^{1 / 10} M\left(\|v\|_{L^{2}\left(H^{5 / 2+1 / 8}\left(\Omega_{F}\right)\right)}+\|v\|_{C^{0}\left(H^{2}\left(\Omega_{F}\right)\right)}\right) \\
& \leqslant C T^{1 / 10} M\left(\|v\|_{L^{2}\left(H^{5 / 2+1 / 8}\left(\Omega_{F}\right)\right)}+\left(1+\left\|v_{0}\right\|_{H^{2}\left(\Omega_{F}\right)}\right)\left\|V_{0}\right\|_{H^{2}\left(\Omega_{F}\right)}+\left\|\Xi_{1}\right\|_{H^{1}\left(\Omega_{S}\right)}\right. \\
&\left.+\|G\|_{H^{1}\left(L^{2}\left(\Omega_{F}\right)\right) \cap C^{0}\left(L^{2}\left(\Omega_{F}\right)\right)}\right) .
\end{aligned}
$$

Similarly, using (3.73) with 2.27, 2.28, 3.32 and (3.36), we deduce

$$
\begin{aligned}
& \left\|\partial_{t} f_{2}\right\|_{L^{2}\left(L^{3 / 2}\left(\Omega_{F}\right)\right)} \\
& \quad \leqslant C M\left(\left(1+\left\|v_{0}\right\|_{H^{2}\left(\Omega_{F}\right)}\right)\left\|V_{0}\right\|_{H^{2}\left(\Omega_{F}\right)}+\left\|\Xi_{1}\right\|_{H^{1}\left(\Omega_{S}\right)}+\|G\|_{H^{1}\left(L^{2}\left(\Omega_{F}\right)\right) \cap C^{0}\left(L^{2}\left(\Omega_{F}\right)\right)}\right) .
\end{aligned}
$$

Thus, (3.70 becomes

$$
\begin{aligned}
\|W\|_{H^{1}\left(L^{2}\left(\Omega_{F}\right)\right)} \leqslant & C M\left(T^{1 / 10} M\|v\|_{L^{2}\left(H^{5 / 2+1 / 8}\left(\Omega_{F}\right)\right)}\right. \\
& \left.+\left(1+\left\|v_{0}\right\|_{H^{2}\left(\Omega_{F}\right)}\right)\left\|V_{0}\right\|_{H^{2}\left(\Omega_{F}\right)}+\left\|\Xi_{1}\right\|_{H^{1}\left(\Omega_{S}\right)}+\|G\|_{H^{1}\left(L^{2}\left(\Omega_{F}\right)\right) \cap C^{0}\left(L^{2}\left(\Omega_{F}\right)\right)}\right) .
\end{aligned}
$$

Moreover, since $f_{2}(0, \cdot)=\nabla V_{0}:\left(\nabla v_{0}\right)^{*}$, we have

$$
\|W(0, \cdot)\|_{L^{2}\left(\Omega_{F}\right)} \leqslant C\left\|v_{0}\right\|_{H^{2}\left(\Omega_{F}\right)}\left\|V_{0}\right\|_{H^{2}\left(\Omega_{F}\right)} .
$$

It remains to estimate the norms of $f_{1}$ in $L^{2}\left(L^{2}\left(\Omega_{F}\right)\right)$ and of $f_{3}$ in $L^{2}\left((0, T) \times \partial \Omega_{S}\right)$ in 3.69$)$. To estimate $\Sigma\left(\partial_{t} \xi\right) n$ that appears in $f_{3}$, we first combine 1.19 , a trace theorem and 3.32 to deduce that

$$
\left\|\partial_{t} \xi\right\|_{H^{1}\left((0, T) \times \partial \Omega_{S}\right)} \leqslant C\left(\left(1+\left\|v_{0}\right\|_{H^{2}\left(\Omega_{F}\right)}\right)\left\|V_{0}\right\|_{H^{2}\left(\Omega_{F}\right)}+\left\|\Xi_{1}\right\|_{H^{1}\left(\Omega_{S}\right)}+\|G\|_{H^{1}\left(L^{2}\left(\Omega_{F}\right)\right) \cap C^{0}\left(L^{2}\left(\Omega_{F}\right)\right)}\right) .
$$

Then, we apply Theorem 3.2 in 25 , to the system satisfied by $\partial_{t} \xi$ :

$$
\Sigma\left(\partial_{t} \xi\right) n \in L^{2}\left((0, T) \times \partial \Omega_{S}\right)
$$

with

$$
\left\|\Sigma\left(\partial_{t} \xi\right) n\right\|_{L^{2}\left((0, T) \times \partial \Omega_{S}\right)} \leqslant C\left(\left(1+\left\|v_{0}\right\|_{H^{2}\left(\Omega_{F}\right)}\right)\left\|V_{0}\right\|_{H^{2}\left(\Omega_{F}\right)}+\left\|\Xi_{1}\right\|_{H^{1}\left(\Omega_{S}\right)}+\|\|_{H^{1}\left(L^{2}\left(\Omega_{F}\right)\right) \cap C^{0}\left(L^{2}\left(\Omega_{F}\right)\right)}\right) .
$$

Moreover, using 3.71, 2.19, 2.24, 2.27, 2.29, we get

$$
\begin{aligned}
\|r\|_{L^{2}\left(0, T, H^{1}\left(\Omega_{F}\right)\right)} \leqslant C T^{1 / 10} M\left(\|v\|_{C^{0}\left(H^{2}\left(\Omega_{F}\right)\right)}+\|q\|_{C^{0}\left(H^{1}\left(\Omega_{F}\right)\right)}\right. & \\
& \left.+\|v\|_{L^{2}\left(H^{5 / 2+1 / 8}\left(\Omega_{F}\right)\right)}+\|q\|_{L^{2}\left(H^{3 / 2+1 / 8}\left(\Omega_{F}\right)\right)}\right) .
\end{aligned}
$$

Using 3.32 and 3.78 , we deduce from the above relation that

$$
\begin{aligned}
\left\|f_{1}\right\|_{L^{2}\left(L^{2}\left(\Omega_{F}\right)\right)} \leqslant C\left(\left\|V_{0}\right\|_{H^{2}\left(\Omega_{F}\right)}+\right. & \left.\left\|\Xi_{1}\right\|_{H^{1}\left(\Omega_{S}\right)}+\|G\|_{H^{1}\left(L^{2}\left(\Omega_{F}\right)\right) \cap C^{0}\left(L^{2}\left(\Omega_{F}\right)\right)}\right) \\
& +C T^{1 / 10} M\left(\|v\|_{L^{2}\left(H^{5 / 2+1 / 8}\left(\Omega_{F}\right)\right)}+\|q\|_{L^{2}\left(H^{3 / 2+1 / 8}\left(\Omega_{F}\right)\right)}\right)
\end{aligned}
$$


and

$$
\begin{array}{r}
\left\|f_{3}\right\|_{L^{2}\left((0, T) \times \partial \Omega_{S}\right)} \leqslant C\left(\left(1+\left\|v_{0}\right\|_{H^{2}\left(\Omega_{F}\right)}\right)\left\|V_{0}\right\|_{H^{2}\left(\Omega_{F}\right)}+\left\|\Xi_{1}\right\|_{H^{1}\left(\Omega_{S}\right)}+\|G\|_{H^{1}\left(L^{2}\left(\Omega_{F}\right)\right) \cap C^{0}\left(L^{2}\left(\Omega_{F}\right)\right)}\right) \\
+C T^{1 / 10} M\left(\|v\|_{L^{2}\left(H^{5 / 2+1 / 8}\left(\Omega_{F}\right)\right)}+\|q\|_{L^{2}\left(H^{3 / 2+1 / 8}\left(\Omega_{F}\right)\right)}\right) .
\end{array}
$$

Inserting 3.76, 3.77), these last two estimates and 3.11) in 3.69), we get the result.

Using Proposition 3.5, we will prove a regularity result on the pressure. The regularity of the pressure in $H^{1 / 3}\left(H^{1}\left(\Omega_{F}\right)\right)$ will be useful in Section 4 .

Proposition 3.6. We have

$$
q \in H^{1 / 3}\left(H^{1}\left(\Omega_{F}\right)\right)
$$

with the estimate

$$
\begin{aligned}
\|q\|_{H^{1 / 3}\left(H^{1}\left(\Omega_{F}\right)\right)} & \leqslant C T^{1 / 10} M\left(\|v\|_{L^{2}\left(H^{5 / 2+1 / 8}\left(\Omega_{F}\right)\right)}+\|q\|_{L^{2}\left(H^{3 / 2+1 / 8}\left(\Omega_{F}\right)\right)}\right) \\
+ & C\left(\left(1+\left\|v_{0}\right\|_{H^{2}\left(\Omega_{F}\right)}\right)\left\|V_{0}\right\|_{H^{2}\left(\Omega_{F}\right)}+\left\|\Xi_{1}\right\|_{H^{1}\left(\Omega_{S}\right)}+\|G\|_{H^{1}\left(L^{2}\left(\Omega_{F}\right)\right) \cap C^{0}\left(L^{2}\left(\Omega_{F}\right)\right)}\right) .
\end{aligned}
$$

Proof. We recall that $(v, q)$ satisfies the elliptic system (3.23), where $R$ is defined in (3.24). We need to estimate the terms appearing in this system.

First, using 2.19, 2.27, 2.29, and Lemma A.7 for $s=1 / 3$ and $\sigma_{0}=1$, we deduce

$$
\|R\|_{H^{1 / 3}\left(H^{1}\left(\Omega_{F}\right)\right)}+\|\nabla v:[\operatorname{Cof}(\nabla \widehat{X})-I]\|_{H^{1 / 3}\left(H^{1}\left(\Omega_{F}\right)\right)} \leqslant C T^{1 / 6} M\left(\|v\|_{H^{1 / 3}\left(H^{2}\left(\Omega_{F}\right)\right)}+\|q\|_{H^{1 / 3}\left(H^{1}\left(\Omega_{F}\right)\right)}\right) .
$$

On the other hand, combining $1.19,3.32$ and the trace theorem, we obtain

$$
\begin{aligned}
\|\xi\|_{H^{2}\left((0, T) \times \partial \Omega_{S}\right)} \leqslant & C T^{1 / 2}\|v\|_{L^{2}\left(H^{5 / 2+1 / 8}\left(\Omega_{F}\right)\right)} \\
& +C\left(\left(1+\left\|v_{0}\right\|_{H^{2}\left(\Omega_{F}\right)}\right)\left\|V_{0}\right\|_{H^{2}\left(\Omega_{F}\right)}+\left\|\Xi_{1}\right\|_{H^{1}\left(\Omega_{S}\right)}+\|G\|_{H^{1}\left(L^{2}\left(\Omega_{F}\right)\right) \cap C^{0}\left(L^{2}\left(\Omega_{F}\right)\right)}\right) .
\end{aligned}
$$

Using [25, Theorem 3.2], we deduce

$$
\begin{aligned}
\|\Sigma(\xi)\|_{H^{1}\left((0, T) \times \partial \Omega_{S}\right)} & \leqslant C T^{1 / 2}\|v\|_{L^{2}\left(H^{5 / 2+1 / 8}\left(\Omega_{F}\right)\right)} \\
+ & +C\left(\left(1+\left\|v_{0}\right\|_{H^{2}\left(\Omega_{F}\right)}\right)\left\|V_{0}\right\|_{H^{2}\left(\Omega_{F}\right)}+\left\|\Xi_{1}\right\|_{H^{1}\left(\Omega_{S}\right)}+\|G\|_{H^{1}\left(L^{2}\left(\Omega_{F}\right)\right) \cap C^{0}\left(L^{2}\left(\Omega_{F}\right)\right)}\right) .
\end{aligned}
$$

Applying Lemma A.5 with $s=1 / 3$ (and where $\Omega$ is replaced by $\partial \Omega_{S}$ ), we deduce from the above estimate

$$
\begin{aligned}
\|\Sigma(\xi)\|_{H^{1 / 3}\left(H^{1 / 2}\left(\partial \Omega_{S}\right)\right)} \leqslant C\|\Sigma(\xi)\|_{H^{1 / 3}\left(H^{2 / 3}\left(\partial \Omega_{S}\right)\right)} \leqslant C T^{1 / 2}\|v\|_{L^{2}\left(H^{5 / 2+1 / 8}\left(\Omega_{F}\right)\right)} \\
+C\left(\left(1+\left\|v_{0}\right\|_{H^{2}\left(\Omega_{F}\right)}\right)\left\|V_{0}\right\|_{H^{2}\left(\Omega_{F}\right)}+\left\|\Xi_{1}\right\|_{H^{1}\left(\Omega_{S}\right)}+\|G\|_{H^{1}\left(L^{2}\left(\Omega_{F}\right)\right) \cap C^{0}\left(L^{2}\left(\Omega_{F}\right)\right)}\right) .
\end{aligned}
$$

We apply elliptic estimates to the system (3.23) satisfied by $(v, q)$ and the estimate (3.81) then follows from 3.82, 3.83 and 3.64). This finishes the proof of Proposition 3.6

At this step, we are in position to complete the proof of Theorem 1.4 in the case of smooth data (that is, data satisfying (2.1)-(2.4). We recall that we have already obtained 3.32 .

From Lemma A.9 (3.64) and 3.32, we have

$$
\partial_{t} v \in H^{1 / 8}\left(L^{2}\left(\partial \Omega_{S}\right)\right)
$$

with the estimate

$$
\begin{aligned}
\left\|\partial_{t} v\right\|_{H^{1 / 8}\left(L^{2}\left(\partial \Omega_{S}\right)\right)} \leqslant C\left(\left(1+\left\|v_{0}\right\|_{H^{2}\left(\Omega_{F}\right)}\right)\right. & \left.\left\|V_{0}\right\|_{H^{2}\left(\Omega_{F}\right)}+\left\|\Xi_{1}\right\|_{H^{1}\left(\Omega_{S}\right)}+\|G\|_{H^{1}\left(L^{2}\left(\Omega_{F}\right)\right) \cap C^{0}\left(L^{2}\left(\Omega_{F}\right)\right)}\right) \\
& +C T^{1 / 10} M\left(\|v\|_{L^{2}\left(H^{5 / 2+1 / 8}\left(\Omega_{F}\right)\right)}+\|q\|_{L^{2}\left(H^{3 / 2+1 / 8}\left(\Omega_{F}\right)\right)}\right) .
\end{aligned}
$$

Since $\partial_{t} \xi=v$ on $(0, T) \times \partial \Omega_{S}$, we have

$$
\|\xi\|_{C^{0}\left(H^{2+1 / 8}\left(\partial \Omega_{S}\right)\right)} \leqslant C T^{1 / 2}\|v\|_{L^{2}\left(H^{2+1 / 8}\left(\partial \Omega_{S}\right)\right)}
$$


and these two estimates yield

$$
\begin{array}{r}
\|\xi\|_{H^{2+1 / 8}\left((0, T) \times \partial \Omega_{S}\right)} \leqslant C\left(\left(1+\left\|v_{0}\right\|_{H^{2}\left(\Omega_{F}\right)}\right)\left\|V_{0}\right\|_{H^{2}\left(\Omega_{F}\right)}+\left\|\Xi_{1}\right\|_{H^{1}\left(\Omega_{S}\right)}+\|G\|_{H^{1}\left(L^{2}\left(\Omega_{F}\right)\right) \cap C^{0}\left(L^{2}\left(\Omega_{F}\right)\right)}\right) \\
+C\left(T^{1 / 10} M+T^{1 / 2}\right)\left(\|v\|_{L^{2}\left(H^{5 / 2+1 / 8}\left(\Omega_{F}\right)\right)}+\|q\|_{L^{2}\left(H^{3 / 2+1 / 8}\left(\Omega_{F}\right)\right)}\right) .
\end{array}
$$

Applying to $\xi$ Theorem 3.2 in 25] combined with interpolation arguments, we deduce

$$
\begin{aligned}
& \|\xi\|_{C^{0}\left(H^{2+1 / 8}\left(\Omega_{S}\right)\right)}+\|\xi\|_{C^{1}\left(H^{1+1 / 8}\left(\Omega_{S}\right)\right)}+\|\Sigma(\xi) n\|_{\left.H^{1+1 / 8}\left((0, T) \times \partial \Omega_{S}\right)\right)} \\
& \leqslant C\left(\left(1+\left\|v_{0}\right\|_{H^{2}\left(\Omega_{F}\right)}\right)\left\|V_{0}\right\|_{H^{2}\left(\Omega_{F}\right)}+\left\|\Xi_{1}\right\|_{H^{1+1 / 8}\left(\Omega_{S}\right)}+\|G\|_{H^{1}\left(L^{2}\left(\Omega_{F}\right)\right) \cap C^{0}\left(L^{2}\left(\Omega_{F}\right)\right)}\right) \\
& \quad+C\left(T^{1 / 10} M+T^{1 / 2}\right)\left(\|v\|_{L^{2}\left(H^{5 / 2+1 / 8}\left(\Omega_{F}\right)\right)}+\|q\|_{L^{2}\left(H^{3 / 2+1 / 8}\left(\Omega_{F}\right)\right)}\right) .
\end{aligned}
$$

We are going now to use the elliptic regularity of the Stokes system 3.23 . From 2.20 , we deduce

$$
\left\|\nabla v:\left[\operatorname{Cof}(\nabla \widehat{X})-I_{3}\right]\right\|_{L^{2}\left(H^{3 / 2+1 / 8}\left(\Omega_{F}\right)\right)} \leqslant C T^{1 / 2} M\|v\|_{L^{2}\left(H^{5 / 2+1 / 8}\left(\Omega_{F}\right)\right.} .
$$

Similarly, from the definition 3.24 of $R$,

$$
\|R\|_{L^{2}\left(H^{3 / 2+1 / 8}\left(\Omega_{F}\right)\right)} \leqslant C T^{1 / 2} M\left(\|v\|_{L^{2}\left(H^{5 / 2+1 / 8}\left(\Omega_{F}\right)\right)}+\|q\|_{L^{2}\left(H^{3 / 2+1 / 8}\left(\Omega_{F}\right)\right)}\right) .
$$

Then from 3.32,

$$
\left\|\partial_{t} v\right\|_{L^{2}\left(H^{1 / 2+1 / 8}\left(\Omega_{F}\right)\right)} \leqslant C\left(\left(1+\left\|v_{0}\right\|_{H^{2}\left(\Omega_{F}\right)}\right)\left\|V_{0}\right\|_{H^{2}\left(\Omega_{F}\right)}+\left\|\Xi_{1}\right\|_{H^{1}\left(\Omega_{S}\right)}+\|G\|_{H^{1}\left(L^{2}\left(\Omega_{F}\right)\right) \cap C^{0}\left(L^{2}\left(\Omega_{F}\right)\right)}\right) .
$$

From the elliptic regularity of the Stokes system (3.23) together with 3.84, (3.85), (3.86) and (3.87), we deduce

$$
\begin{aligned}
& \|v\|_{L^{2}\left(H^{5 / 2+1 / 8}\left(\Omega_{F}\right)\right)}+\|q\|_{L^{2}\left(H^{3 / 2+1 / 8}\left(\Omega_{F}\right)\right)} \\
\leqslant & C\left(\left(1+\left\|v_{0}\right\|_{H^{2}\left(\Omega_{F}\right)}\right)\left\|V_{0}\right\|_{H^{2}\left(\Omega_{F}\right)}+\left\|\Xi_{1}\right\|_{H^{1+1 / 8}\left(\Omega_{S}\right)}+\|G\|_{H^{1}\left(L^{2}\left(\Omega_{F}\right)\right) \cap C^{0}\left(L^{2}\left(\Omega_{F}\right)\right) \cap L^{2}\left(H^{1 / 2+1 / 8}\left(\Omega_{F}\right)\right)}\right) .
\end{aligned}
$$

Going back to (3.84, this allows us to deduce 1.28 if the data satisfy $2.1-2.4$.

\subsection{A density argument}

We are now in position to conclude the proof of Theorem 1.4

Let us consider $\left(V_{0}, \Xi_{1}, G\right)$ satisfying $(1.23)-(1.27)$. Using Section B there exists a sequence $\left(\left(V_{0}^{k}, \Xi_{1}^{k}, G^{k}\right)\right)_{k}$ satisfying (2.1)-(2.4) and such that

$$
\begin{gathered}
V_{0}^{k} \rightarrow V_{0} \quad \text { in } \quad H^{2}\left(\Omega_{F}\right), \\
\Xi_{1}^{k} \rightarrow \Xi_{1} \quad \text { in } \quad H^{1+1 / 8}\left(\Omega_{S}\right), \\
G^{k} \rightarrow G \quad \text { in } \quad H^{1}\left(L^{2}\left(\Omega_{F}\right)\right) \cap L^{2}\left(H^{1 / 2+1 / 8}\left(\Omega_{F}\right)\right) .
\end{gathered}
$$

For all $k$, we can consider the solution $\left(X^{k}, v^{k}, q^{k}, \xi^{k}\right)$ of the system (1.14)-(1.22 associated with $\left(V_{0}^{k}, \Xi_{1}^{k}, G^{k}\right)$ that we can construct as above in the interval $(0, T)$ with $T$ satisfying $(2.6)$ for some $\alpha$ and $C$.

From 1.28, we deduce that $\left(\left(X^{k}, v^{k}, q^{k}, \xi^{k}\right)\right)_{k}$ is a Cauchy sequence in $\mathcal{S}_{1, T} \times \mathcal{S}_{2, T} \times \mathcal{S}_{3, T} \times \mathcal{S}_{4, T}$. It thus converges to $(X, v, q, \xi) \in \mathcal{S}_{1, T} \times \mathcal{S}_{2, T} \times \mathcal{S}_{3, T} \times \mathcal{S}_{4, T}$ and we have

$$
\left(X^{k}, v^{k}, q^{k}, \xi^{k}\right) \rightarrow(X, v, q, \xi) \quad \text { in } \mathcal{S}_{1, T} \times \mathcal{S}_{2, T} \times \mathcal{S}_{3, T} \times \mathcal{S}_{4, T}
$$

Using the linearity of the system $1.14-1.22$, we deduce that $(X, v, q, \xi)$ is the solution of $1.14-(1.22$. Moreover it satisfies (1.28). 


\section{Proof of Theorem 1.1}

We recall that $\left(v_{0}, \xi_{1}, g\right)$ satisfies $1.7-1.9$. From Theorem 1.4 there exist $\alpha>0$ and $C>0$ such that for all $T$ satisfying $(2.6)$ and for all $\widehat{X} \in B_{M}$ (see (1.11), there exists a unique solution $(X, v, q, \xi)$ of the system 1.14-1.22 where

$$
V_{0}=v_{0}, \quad \Xi_{1}=\xi_{1} \quad G=g .
$$

Using (1.28), we can choose $M$ large enough (and $T$ satisfying 2.6) such that the mapping

$$
\Lambda: \widehat{X} \in B_{M} \mapsto X \in B_{M}
$$

is well-defined.

Our goal now is to show that $\Lambda$ has a fixed point. This is done by proving that, for some $\alpha>0$

$$
\left\|\Lambda\left(\widehat{X}^{1}\right)-\Lambda\left(\widehat{X}^{2}\right)\right\|_{H^{1}\left(H^{2}\left(\Omega_{F}\right)\right) \cap H^{2}\left(L^{2}\left(\Omega_{F}\right)\right)} \leqslant C T^{\alpha}\left\|\widehat{X}^{1}-\widehat{X}^{2}\right\|_{H^{1}\left(H^{2}\left(\Omega_{F}\right)\right) \cap H^{2}\left(L^{2}\left(\Omega_{F}\right)\right)}
$$

that yields that, for $T$ small, $\Lambda$ is a contraction for the topology of $H^{1}\left(H^{2}\left(\Omega_{F}\right)\right) \cap H^{2}\left(L^{2}\left(\Omega_{F}\right)\right)$. Since $B_{M}$ is a closed set of $H^{1}\left(H^{2}\left(\Omega_{F}\right)\right) \cap H^{2}\left(L^{2}\left(\Omega_{F}\right)\right)$, we deduce that $\Lambda$ admits a unique fixed point in $B_{M}$.

Let us consider $\widehat{X}^{1}, \widehat{X}^{2} \in B_{M}$ (see $(1.11)$ ). For $i=1,2$, we denote by $\left(X^{i}, v^{i}, q^{i}, \xi^{i}\right)$ the unique solution of the system $1.14-(1.20)$ with 4.1 .

We set

$$
v:=v^{1}-v^{2}, \quad q:=q^{1}-q^{2}, \quad \xi:=\xi^{1}-\xi^{2} .
$$

Then

$$
\begin{cases}\partial_{t} v-\operatorname{div} \mathbb{T}_{\widehat{X}^{1}}(v, q)=-\operatorname{div} \mathbb{T}_{\widehat{X}^{2}}\left(v^{2}, q^{2}\right)+\operatorname{div} \mathbb{T}_{\widehat{X}^{1}}\left(v^{2}, q^{2}\right) & \text { in }(0, T) \times \Omega_{F}, \\ \nabla v: \operatorname{Cof}\left(\nabla \widehat{X}^{1}\right)=\nabla v^{2}:\left[\operatorname{Cof}\left(\nabla \widehat{X}^{2}\right)-\operatorname{Cof}\left(\nabla \widehat{X}^{1}\right)\right] & \text { in }(0, T) \times \Omega_{F}, \\ v=0 & \text { on }(0, T) \times \partial \Omega, \\ \mathbb{T}_{\widehat{X}^{1}}(v, q) n=\Sigma(\xi) n+\mathbb{T}_{\widehat{X}^{2}}\left(v^{2}, q^{2}\right) n-\mathbb{T}_{\widehat{X}^{1}}\left(v^{2}, q^{2}\right) n & \text { on }(0, T) \times \partial \Omega_{S}, \\ v(0, \cdot)=0 & \text { in } \Omega_{F}\end{cases}
$$

and

$$
\left\{\begin{array}{l}
\partial_{t t} \xi-\operatorname{div} \Sigma(\xi)=0 \quad \text { in }(0, T) \times \Omega_{S} \\
v=\partial_{t} \xi \quad \text { in }(0, T) \times \partial \Omega_{S} \\
\xi(0, \cdot)=0, \partial_{t} \xi(0, \cdot)=0 \quad \text { in } \Omega_{S}
\end{array}\right.
$$

We first remark that to prove (4.3), it is sufficient to prove the following estimate: there exists $\alpha>0$ such that

$$
\|v\|_{L^{2}\left(H^{2}\left(\Omega_{F}\right)\right)}+\|v\|_{H^{1}\left(L^{2}\left(\Omega_{F}\right)\right)} \leqslant C T^{\alpha}\left(\left\|\widehat{v}^{1}-\widehat{v}^{2}\right\|_{L^{2}\left(H^{2}\left(\Omega_{F}\right)\right)}+\left\|\widehat{v}^{1}-\widehat{v}^{2}\right\|_{H^{1}\left(L^{2}\left(\Omega_{F}\right)\right)}\right) .
$$

Using Lemma 3.1 and assuming (2.6), we deduce

$$
\begin{array}{r}
\|v\|_{L^{2}\left(H^{2}\left(\Omega_{F}\right)\right) \cap H^{1}\left(L^{2}\left(\Omega_{F}\right)\right)} \leqslant C\left(\left\|-\operatorname{div} \mathbb{T}_{\widehat{X}^{2}}\left(v^{2}, q^{2}\right)+\operatorname{div} \mathbb{T}_{\widehat{X}^{1}}\left(v^{2}, q^{2}\right)\right\|_{L^{2}\left(L^{2}\left(\Omega_{F}\right)\right)}\right. \\
+\left\|\nabla v^{2}:\left[\operatorname{Cof}\left(\nabla \widehat{X}^{2}\right)-\operatorname{Cof}\left(\nabla \widehat{X}^{1}\right)\right]\right\|_{H^{1}\left(H^{-1}\left(\Omega_{F}\right)\right) \cap L^{2}\left(H^{1}\left(\Omega_{F}\right)\right)} \\
+\left\|\mathbb{T}_{\widehat{X}^{2}}\left(v^{2}, q^{2}\right) n-\mathbb{T}_{\widehat{X}^{1}}\left(v^{2}, q^{2}\right) n\right\|_{L^{2}\left(H^{1 / 2}\left(\partial \Omega_{S}\right)\right) \cap H^{1 / 4}\left(L^{2}\left(\partial \Omega_{S}\right)\right)} \\
\left.+\|\Sigma(\xi) n\|_{L^{2}\left(H^{1 / 2}\left(\partial \Omega_{S}\right)\right) \cap H^{1 / 4}\left(L^{2}\left(\partial \Omega_{S}\right)\right)}\right) .
\end{array}
$$

First, we have

$$
\left\|\nabla \widehat{X}^{1}-\nabla \widehat{X}^{2}\right\|_{C^{0}\left(H^{1}\left(\Omega_{F}\right)\right)} \leqslant T^{1 / 2}\left\|\widehat{v}^{1}-\widehat{v}^{2}\right\|_{L^{2}\left(H^{2}\left(\Omega_{F}\right)\right)}
$$

Using this estimate combined with 1.28, 1.6 and 1.27, we deduce that

$$
\begin{gathered}
\left\|-\operatorname{div} \mathbb{T}_{\widehat{X}^{2}}\left(v^{2}, q^{2}\right)+\operatorname{div} \mathbb{T}_{\widehat{X}^{1}}\left(v^{2}, q^{2}\right)\right\|_{L^{2}\left(L^{2}\left(\Omega_{F}\right)\right)} \leqslant C T^{\alpha}\left\|\widehat{v}^{1}-\widehat{v}^{2}\right\|_{L^{2}\left(H^{2}\left(\Omega_{F}\right)\right)}, \\
\left\|\nabla v^{2}:\left[\operatorname{Cof}\left(\nabla \widehat{X}^{2}\right)-\operatorname{Cof}\left(\nabla \widehat{X}^{1}\right)\right]\right\|_{L^{2}\left(H^{1}\left(\Omega_{F}\right)\right)} \leqslant C T^{\alpha}\left\|\widehat{v}^{1}-\widehat{v}^{2}\right\|_{L^{2}\left(H^{2}\left(\Omega_{F}\right)\right)}, \\
\left\|\mathbb{T}_{\widehat{X}^{2}}\left(v^{2}, q^{2}\right) n-\mathbb{T}_{\widehat{X}^{1}}\left(v^{2}, q^{2}\right) n\right\|_{L^{2}\left(H^{1 / 2}\left(\partial \Omega_{S}\right)\right)} \leqslant C T^{\alpha}\left\|\widehat{v}^{1}-\widehat{v}^{2}\right\|_{L^{2}\left(H^{2}\left(\Omega_{F}\right)\right)},
\end{gathered}
$$


for some $\alpha>0$. According to Lemma A.6 with $p_{1}=2, p_{2}=\infty, m_{1}=2$ and $m_{2}=0$

$$
\left\|\widehat{v}^{1}-\widehat{v}^{2}\right\|_{L^{8 / 3}\left(H^{3 / 2}\left(\Omega_{F}\right)\right)} \leqslant C\left\|\widehat{v}^{1}-\widehat{v}^{2}\right\|_{L^{\infty}\left(L^{2}\left(\Omega_{F}\right)\right) \cap L^{2}\left(H^{2}\left(\Omega_{F}\right)\right)} .
$$

We deduce that

$$
\left\|\nabla \widehat{v}^{1}-\nabla \widehat{v}^{2}\right\|_{L^{2}\left(L^{3}\left(\Omega_{F}\right)\right)} \leqslant C T^{1 / 8}\left\|\widehat{v}^{1}-\widehat{v}^{2}\right\|_{L^{\infty}\left(L^{2}\left(\Omega_{F}\right)\right) \cap L^{2}\left(H^{2}\left(\Omega_{F}\right)\right)} .
$$

Using this estimate, the fact that $L^{6 / 5}\left(\Omega_{F}\right) \subset H^{-1}\left(\Omega_{F}\right)$ and 4.8 , we deduce

$$
\begin{aligned}
& \left\|\nabla v^{2}:\left[\operatorname{Cof}\left(\nabla \widehat{X}^{2}\right)-\operatorname{Cof}\left(\nabla \widehat{X}^{1}\right)\right]\right\|_{H^{1}\left(H^{-1}\left(\Omega_{F}\right)\right)} \\
& \quad \leqslant C T^{\alpha}\left(\left\|\widehat{v}^{1}-\widehat{v}^{2}\right\|_{L^{2}\left(H^{2}\left(\Omega_{F}\right)\right)}+\left\|\widehat{v}^{1}-\widehat{v}^{2}\right\|_{H^{1}\left(L^{2}\left(\Omega_{F}\right)\right)}\right) .
\end{aligned}
$$

Let us now estimate

$$
\left\|\mathbb{T}_{\widehat{X}^{2}}\left(v^{2}, q^{2}\right) n-\mathbb{T}_{\widehat{X}^{1}}\left(v^{2}, q^{2}\right) n\right\|_{H^{1 / 4}\left(L^{2}\left(\partial \Omega_{S}\right)\right)} .
$$

In order to do this, we first combine 1.28 and Lemma A.5 with $s=1 / 3$ and the spaces $H^{1}\left(L^{2}\left(\Omega_{F}\right)\right)$, $L^{2}\left(H^{3 / 2+1 / 8}\left(\Omega_{F}\right)\right)$ to deduce that

$$
\left\|\nabla v^{2}\right\|_{H^{1 / 3}\left(H^{1}\left(\Omega_{F}\right)\right)}+\left\|q^{2}\right\|_{H^{1 / 3}\left(H^{1}\left(\Omega_{F}\right)\right)} \leqslant C M .
$$

Moreover, for $i=1,2$, we deduce from 2.19

$$
\left\|\nabla \widehat{X}^{i}\right\|_{C^{0}\left(H^{3 / 2+1 / 8}\left(\Omega_{F}\right)\right)}+\left\|\nabla \widehat{v}^{i}\right\|_{L^{2}\left(H^{3 / 2+1 / 8}\left(\Omega_{F}\right)\right)} \leqslant C(M+1),
$$

and, using 4.8, this implies

$$
\left\|\operatorname{Cof}\left(\nabla \widehat{X}^{2}\right)-\operatorname{Cof}\left(\nabla \widehat{X}^{1}\right)\right\|_{H^{1}\left(H^{1}\left(\Omega_{F}\right)\right)} \leqslant C(M+1)\left\|\widehat{v}^{1}-\widehat{v}^{2}\right\|_{L^{2}\left(H^{2}\left(\Omega_{F}\right)\right)} .
$$

We deduce from the above estimates and from Lemma A.7 (with $s=1 / 4, \sigma_{0}=1$ ) that

$$
\left\|\mathbb{T}_{\widehat{X}^{2}}\left(v^{2}, q^{2}\right)-\mathbb{T}_{\widehat{X}^{1}}\left(v^{2}, q^{2}\right)\right\|_{H^{1 / 3}\left(H^{1}\left(\Omega_{F}\right)\right)} \leqslant C T^{\alpha}\left\|\widehat{v}^{1}-\widehat{v}^{2}\right\|_{L^{2}\left(H^{2}\left(\Omega_{F}\right)\right)} .
$$

The above estimate and the trace theorem yield

$$
\left\|\mathbb{T}_{\widehat{X}^{2}}\left(v^{2}, q^{2}\right) n-\mathbb{T}_{\widehat{X}^{1}}\left(v^{2}, q^{2}\right) n\right\|_{H^{1 / 3}\left(L^{2}\left(\partial \Omega_{S}\right)\right)} \leqslant C T^{\alpha}\left\|\widehat{v}^{1}-\widehat{v}^{2}\right\|_{L^{2}\left(H^{2}\left(\Omega_{F}\right)\right)} .
$$

In order to estimate the last term in the right-hand side of (4.7), we use Corollary A.3 with $\left(s_{2}, \sigma_{2}\right)=$ $(3 / 2,3 / 2+1 / 4)$ and Corollary A.2

$$
\|\xi\|_{L^{2}\left(H^{3 / 2}\left(\partial \Omega_{S}\right)\right) \cap H^{3 / 2}\left(L^{2}\left(\partial \Omega_{S}\right)\right)} \leqslant C\left(T\|v\|_{L^{2}\left(H^{2}\left(\Omega_{F}\right)\right)}+T^{1 / 4}\|v\|_{H^{3 / 4}\left(L^{2}\left(\partial \Omega_{S}\right)\right)}\right) .
$$

Then, Lemma A.8 implies

$$
\|\xi\|_{L^{2}\left(H^{3 / 2}\left(\partial \Omega_{S}\right)\right) \cap H^{3 / 2}\left(L^{2}\left(\partial \Omega_{S}\right)\right)} \leqslant C T^{1 / 4}\left(\|v\|_{L^{2}\left(H^{2}\left(\Omega_{F}\right)\right)}+\|v\|_{H^{1}\left(L^{2}\left(\Omega_{F}\right)\right)}\right) .
$$

Applying Theorem 3.2 in 25] to 4.5 and using interpolation arguments, we deduce

$$
\|\Sigma(\xi) n\|_{L^{2}\left(H^{1 / 2}\left(\partial \Omega_{S}\right)\right) \cap H^{1 / 2}\left(L^{2}\left(\partial \Omega_{S}\right)\right)} \leqslant C T^{1 / 4}\left(\|v\|_{L^{2}\left(H^{2}\left(\Omega_{F}\right)\right)}+\|v\|_{H^{1}\left(L^{2}\left(\Omega_{F}\right)\right)}\right) .
$$

As a conclusion, 4.6 is proved and the proof of Theorem 1.1 is complete.

\section{A Some technical results on Sobolev-Slobodeckij spaces}

We first recall that for $s>3 / 2$,

$$
H^{s}\left(\Omega_{F}\right) \subset L^{\infty}\left(\Omega_{F}\right)
$$

and thus, for any $s \in(3 / 2,2]$, there exists $C=C\left(s, \Omega_{F}\right)>0$ such that, for all $v \in H^{2}\left(\Omega_{F}\right)$

$$
\|v\|_{L^{\infty}\left(\Omega_{F}\right)} \leqslant C\|v\|_{H^{1}\left(\Omega_{F}\right)}^{2-s}\|v\|_{H^{2}\left(\Omega_{F}\right)}^{s-1} .
$$

Assume $\mathcal{X}$ is a Banach space. In this section, we assume

$$
T \leqslant 1
$$


We use the standard notation

$$
\lfloor f\rfloor_{s, 2,(0, T), \mathcal{X}}:=\left(\iint_{(0, T)^{2}} \frac{\left\|f\left(t_{1}, \cdot\right)-f\left(t_{2}, \cdot\right)\right\|_{\mathcal{X}}^{2}}{\left|t_{1}-t_{2}\right|^{2 s+1}} d t_{1} d t_{2}\right)^{\frac{1}{2}} \quad 0<s<1 .
$$

In that case, one can consider the following norm for $H^{s}(0, T ; \mathcal{X})$ :

We define

$$
\begin{gathered}
\|f\|_{H^{s}(0, T ; \mathcal{X})}:=\left(\|f\|_{L^{2}(0, T ; \mathcal{X})}^{2}+\lfloor f\rfloor_{s, 2,(0, T), \mathcal{X}}^{2}\right)^{1 / 2} \quad 0<s<1, \\
\|f\|_{H^{s}(0, T ; \mathcal{X})}:=\left(\|f\|_{H^{1}(0, T ; \mathcal{X})}^{2}+\left\lfloor\partial_{t} f\right\rfloor_{s-1,2,(0, T), \mathcal{X}}^{2}\right)^{1 / 2} \quad 1<s<2 .
\end{gathered}
$$

Then, we have the following relations

$$
\begin{gathered}
\left\|f^{*}\right\|_{L^{2}(0,1 ; \mathcal{X})}^{2}=T^{-1}\|f\|_{L^{2}(0, T ; \mathcal{X})}^{2}, \\
\left\|\partial_{t^{*}} f^{*}\right\|_{L^{2}(0,1 ; \mathcal{X})}^{2}=T\left\|\partial_{t} f\right\|_{L^{2}(0, T ; \mathcal{X})}^{2}, \\
\left\|\partial_{t^{*} t^{*}} f^{*}\right\|_{L^{2}(0,1 ; \mathcal{X})}^{2}=T^{3}\left\|\partial_{t t} f\right\|_{L^{2}(0, T ; \mathcal{X})}^{2}, \\
\left\lfloor f^{*}\right\rfloor_{s, 2,(0,1), \mathcal{X}}^{2}=T^{2 s-1}\lfloor f\rfloor_{s, 2,(0, T), \mathcal{X}}^{2} \quad(0<s<1), \\
\left\lfloor\partial_{t^{*}} f^{*}\right\rfloor_{s-1,2,(0,1), \mathcal{X}}^{2}=T^{2 s-1}\left\lfloor\partial_{t} f\right\rfloor_{s-1,2,(0, T), \mathcal{X}}^{2} \quad(1<s<2) .
\end{gathered}
$$

In particular,

$$
T^{-1 / 2}\|f\|_{H^{s}(0, T ; \mathcal{X})} \geqslant\left\|f^{*}\right\|_{H^{s}(0,1 ; \mathcal{X})} \geqslant T^{s-1 / 2}\|f\|_{H^{s}(0, T ; \mathcal{X})}
$$

for $s \in(0,1)$ and for $T \in(0,1]$.

In this section, we use the notation

$$
\oint f:=\frac{1}{T} \int_{0}^{T} f(s) d s
$$

We define the spaces

$$
H_{L}^{s}(0, T ; \mathcal{X}):=\left\{\begin{array}{l}
\left\{f \in H^{s}(0, T ; \mathcal{X}) ; f(0, \cdot)=0\right\} \quad \text { if } 1 / 2<s<3 / 2 \\
\left\{f \in H^{s}(0, T ; \mathcal{X}) ; f(0, \cdot)=0, \partial_{t} f(0, \cdot)=0\right\} \quad \text { if } 3 / 2<s<5 / 2
\end{array}\right.
$$

and

$$
H_{R}^{s}(0, T ; \mathcal{X}):=\left\{\begin{array}{l}
\left\{f \in H^{s}(0, T ; \mathcal{X}) ; f(T, \cdot)=0\right\} \quad \text { if } 1 / 2<s<3 / 2 \\
\left\{f \in H^{s}(0, T ; \mathcal{X}) ; f(T, \cdot)=0, \partial_{t} f(T, \cdot)=0\right\} \quad \text { if } 3 / 2<s<5 / 2
\end{array}\right.
$$

Lemma A.1. Assume $1 / 2<s<1$. The semi-norm A.2) is a norm in $H_{L}^{s}(0, T ; \mathcal{X})$, equivalent to (A.3). Moreover, there exists $C>0$ such that

$$
\|f\|_{L^{2}(0, T ; \mathcal{X})} \leqslant C T^{s}\lfloor f\rfloor_{s, 2,(0, T), \mathcal{X}} \quad \forall f \in H_{L}^{s}(0, T ; \mathcal{X}) .
$$

The same result holds for $H_{R}^{s}(0, T ; \mathcal{X})$.

Proof. First, we observe that $\lfloor\cdot\rfloor_{s, 2,(0,1), \mathcal{X}}$ is a norm in $H_{L}^{s}(0,1 ; \mathcal{X})$ since

$$
\left\lfloor f^{*}\right\rfloor_{s, 2,(0,1), \mathcal{X}}=0 \Rightarrow f^{*} \text { is constant }
$$

and $f^{*}(0, \cdot)=0$. In order to prove A.12, it suffices to prove

$$
\left\|f^{*}\right\|_{L^{2}(0,1 ; \mathcal{X})} \leqslant C\left\lfloor f^{*}\right\rfloor_{s, 2,(0,1), \mathcal{X}}, \forall f^{*} \in H_{L}^{s}(0,1 ; \mathcal{X}) .
$$

In fact, taking into account A.6 and A.9, we easily find A.12 from A.13. We prove A.13 by contradiction. For each $n \in \mathbb{N}^{*}$ we consider $f_{n}^{*} \in H_{L}^{s}(0,1 ; \mathcal{X})$ such that

$$
\left\|f_{n}^{*}\right\|_{L^{2}(0,1 ; \mathcal{X})}=1 \quad \text { and } \quad\left\lfloor f_{n}^{*}\right\rfloor_{s, 2,(0,1), \mathcal{X}}=\frac{1}{n} .
$$

We set

$$
\ell_{n}^{*}:(0,1) \rightarrow \mathbb{R}, \quad t^{*} \mapsto\left\|f_{n}^{*}\left(t^{*}, \cdot\right)\right\|_{\mathcal{X}}
$$


From A.14 and A.2, we deduce that $\left(\ell_{n}^{*}\right)$ is bounded in $H^{s}(0,1)$. From the compact injection of $H^{s}(0,1)$ in $L^{2}(0,1)$, we deduce that (up to a subsequence),

$$
\ell_{n}^{*} \rightarrow \ell^{*} \text { strongly in } L^{2}(0,1) .
$$

Combining this with $\left\lfloor\ell_{n}^{*}\right\rfloor_{s, 2,(0,1)}=1 / n$, we have that $\left(\ell_{n}^{*}\right)$ is a Cauchy sequence in $H^{s}(0,1)$. Thus, we deduce $\left\lfloor\ell^{*}\right\rfloor_{s, 2,(0,1)}=0$ and $\left\|\ell^{*}\right\|_{L^{2}(0,1)}=1$, but this is absurd since $\ell^{*} \in H_{L}^{s}(0,1)$. Consequently, A.13 is established.

Combining the above result and the Poincaré inequality, we deduce the following result:

Corollary A.2. Assume $3 / 2<s<2$. The semi-norm $f \mapsto\left\lfloor\partial_{t} f\right\rfloor_{s-1,2,(0, T), \mathcal{X}}$ is a norm in $H_{L}^{s}(0, T ; \mathcal{X})$, equivalent to A.4. Moreover, there exists $C>0$ independent of $T$ such that

$$
\|f\|_{H^{1}(0, T ; \mathcal{X})} \leqslant C T^{s-1}\left\lfloor\partial_{t} f\right\rfloor_{s-1,2,(0, T), \mathcal{X}}, \forall f \in H_{L}^{s}(0, T ; \mathcal{X}) .
$$

The same result holds for $H_{R}^{s}(0, T ; \mathcal{X})$.

Finally, we deduce the following result.

Corollary A.3. Assume $1 / 2<\sigma_{1} \leqslant 1$ and $s_{1} \in\left[0, \sigma_{1}\right]$. Then there exists $C>0$ independent of $T$ such that

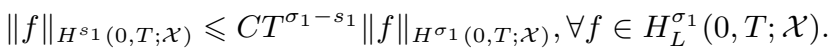

Similarly, assume $3 / 2<\sigma_{2} \leqslant 2$ and $s_{2} \in\left[0, \sigma_{2}\right]$. Then, there exists $C>0$ independent of $T$ such that

$$
\|f\|_{H^{s_{2}(0, T ; \mathcal{X})}} \leqslant C T^{\sigma_{2}-s_{2}}\|f\|_{H^{\sigma_{2}(0, T ; \mathcal{X})}}, \forall f \in H_{L}^{\sigma_{2}}(0, T ; \mathcal{X}) .
$$

The same result holds for $H_{R}^{s}(0, T ; \mathcal{X})$.

Corollary A.4. Assume $s>1 / 2$ and let us endow $H_{L}^{s}(0, T ; \mathcal{X})$ with the norm A.2. Then

$$
\left\|f^{*}\right\|_{\left(H_{L}^{s}\right)^{\prime}(0,1 ; \mathcal{X})}=T^{-s-1 / 2}\|f\|_{\left(H_{L}^{s}\right)^{\prime}\left(0, T ; L^{2}\left(\Omega_{F}\right)\right)} .
$$

Assume $s<1 / 2$. Then

$$
\left\|f^{*}\right\|_{H^{-s}(0,1 ; \mathcal{X})} \leqslant T^{-s-1 / 2}\|f\|_{H^{-s}\left(0, T ; L^{2}\left(\Omega_{F}\right)\right)} .
$$

Proof. First, we notice that for $f, g \in L^{2}(0, T ; \mathcal{X})$,

$$
\int_{0}^{1}\left(f^{*}\left(t^{*}\right), g^{*}\left(t^{*}\right)\right) \mathcal{X} d t^{*}=T^{-1} \int_{0}^{T}(f(t), g(t)) \mathcal{X} d t .
$$

From A.11, this allows to deduce the estimates for $H^{-s}, s<1 / 2$ :

$$
\left\|f^{*}\right\|_{H^{-s}(0,1 ; \mathcal{X})}=\sup _{g^{*} \neq 0} \frac{\left\langle f^{*}, g^{*}\right\rangle}{\left\|g^{*}\right\|_{H^{s}(0,1 ; \mathcal{X})}} \leqslant \sup _{g \neq 0} \frac{T^{-1}\langle f, g\rangle}{T^{s-1 / 2}\|g\|_{H^{s}(0, T ; \mathcal{X})}}=T^{-s-1 / 2}\|f\|_{H^{-s}(0, T ; \mathcal{X})} .
$$

Then for $s>1 / 2$, using Lemma A.1 and A.9, we obtain

$$
\left\|f^{*}\right\|_{\left(H_{L}^{s}\right)^{\prime}(0,1 ; \mathcal{X})}=\sup _{g^{*} \neq 0} \frac{\left\langle f^{*}, g^{*}\right\rangle}{\left\|g^{*}\right\|_{H_{L}^{s}(0,1 ; \mathcal{X})}}=\sup _{g \neq 0} \frac{T^{-1}\langle f, g\rangle}{T^{s-1 / 2}\|g\|_{H_{L}^{s}(0, T ; \mathcal{X})}}=T^{-s-1 / 2}\|f\|_{\left(H_{L}^{s}\right)^{\prime}(0, T ; \mathcal{X})} .
$$

We also show that the interpolation of spaces of the form $H^{s}\left(0, T ; H^{m}(\Omega)\right)$ can be done with constants independent of $T$ :

\section{Lemma A.5.}

1. Let $s \in[0,1], m_{1}, m_{2} \in \mathbb{R}_{+}$and $m:=s m_{1}+(1-s) m_{2}$. Then, there exists a constant $C$ independent of $T$ such that

$$
\|f\|_{H^{s}\left(0, T ; H^{m}(\Omega)\right)} \leqslant C\|f\|_{H^{1}\left(0, T ; H^{m_{1}}(\Omega)\right)}^{s}\|f\|_{L^{2}\left(0, T ; H^{\left.m_{2}(\Omega)\right)}\right.}^{1-s}
$$

for all $f \in H^{1}\left(0, T ; H^{m_{1}}(\Omega)\right) \cap L^{2}\left(0, T ; H^{m_{2}}(\Omega)\right)$. 
2. Let $s \in[1,2], m_{1}, m_{2} \in \mathbb{R}_{+}$and $m:=(s-1) m_{1}+(2-s) m_{2}$. Then, there exists a constant $C$ independent of $T$ such that

$$
\|f\|_{H^{s}\left(0, T ; H^{m}(\Omega)\right)} \leqslant C\|f\|_{H^{2}\left(0, T ; H^{m_{1}}(\Omega)\right)}^{s-1}\|f\|_{H^{1}\left(0, T ; H^{\left.m_{2}(\Omega)\right)}\right.}^{2-s}
$$

for all $f \in H^{2}\left(0, T ; H^{m_{1}}(\Omega)\right) \cap H^{1}\left(0, T ; H^{m_{2}}(\Omega)\right)$.

Proof. Let us start by proving the first assertion: we assume $s \in(0,1)$. By definition, we have

$$
\|f\|_{H^{s}\left(0, T ; H^{m}(\Omega)\right)}^{2}=\|f\|_{L^{2}\left(0, T ; H^{m}(\Omega)\right)}^{2}+\lfloor f\rfloor_{s, 2,(0, T), H^{m}(\Omega)}^{2} .
$$

For the first term of this expression, we simply have

$$
\|f\|_{L^{2}\left(0, T ; H^{m}(\Omega)\right)}^{2} \leqslant C\|f\|_{L^{2}\left(0, T ; H^{\left.m_{2}(\Omega)\right)}\right.}^{2-2 s}\|f\|_{L^{2}\left(0, T ; H^{m_{1}}(\Omega)\right)}^{2 s} \leqslant C\|f\|_{L^{2}\left(0, T ; H^{\left.m_{2}(\Omega)\right)}\right.}^{2-2 s}\|f\|_{H^{1}\left(0, T ; H^{\left.m_{1}(\Omega)\right)}\right.}^{2 s},
$$

where $C$ is independent of $T$. Then,

$$
\begin{aligned}
& \lfloor f\rfloor_{s, 2,(0, T), H^{m}(\Omega)}^{2}=T^{1-2 s}\left\lfloor f^{*}\right\rfloor_{s, 2,(0,1), H^{m}(\Omega)}^{2}=T^{1-2 s}\left\lfloor f^{*}-\oint f^{*}\right\rfloor_{s, 2,(0,1), H^{m}(\Omega)}^{2} \\
& \leqslant T^{1-2 s}\left\|f^{*}-\oint f^{*}\right\|_{H^{s}\left(0,1 ; H^{m}(\Omega)\right)}^{2} \leqslant C T^{1-2 s}\left\|f^{*}-\oint f^{*}\right\|_{L^{2}\left(0,1 ; H^{\left.m_{2}(\Omega)\right)}\right.}^{2-2 s}\left\|f^{*}-\oint f^{*}\right\|_{H^{1}\left(0,1 ; H^{\left.m_{1}(\Omega)\right)}\right.}^{2 s} \\
& \leqslant C T^{1-2 s}\left\|f^{*}-\oint f^{*}\right\|_{L^{2}\left(0,1 ; H^{m_{2}(\Omega)}\right)}^{2-2 s}\left\|\partial_{t^{*}} f^{*}\right\|_{L^{2}\left(0,1 ; H^{m_{1}}(\Omega)\right)}^{2 s} \\
& =C T^{1-2 s} T^{s-1}\|f-\oint f\|_{L^{2}\left(0, T ; H^{\left.m_{2}(\Omega)\right)}\right.}^{2-2 s} T^{s}\left\|\partial_{t} f\right\|_{L^{2}\left(0, T ; H^{\left.m_{1}(\Omega)\right)}\right.}^{2 s} \leqslant C\|f\|_{L^{2}\left(0, T ; H^{\left.m_{2}(\Omega)\right)}\right.}^{2-2 s}\|f\|_{H^{1}\left(0, T ; H^{\left.m_{1}(\Omega)\right)}\right.}^{2 s}
\end{aligned}
$$

For the last inequality, we have used the fact that

$$
\|\oint f\|_{L^{2}\left(0, T ; H^{\left.m_{2}(\Omega)\right)}\right.} \leqslant\|f\|_{L^{2}\left(0, T ; H^{\left.m_{2}(\Omega)\right)} .\right.}
$$

As long as the second assertion is concerned, it suffices to recall the definition of the $H^{s}\left(0, T ; H^{m}(\Omega)\right)$ norm and use A.16 for $\partial_{t} f$ in order to estimate $\left\lfloor\partial_{t} f\right\rfloor_{s, 2,(0, T)}^{2}$.

Using the Hölder inequality, we can deduce the following result.

Lemma A.6. Let $p_{1}, p_{2} \in[1,+\infty], m_{1}, m_{2} \in \mathbb{R}_{+}, \theta \in[0,1]$ and

$$
\frac{1}{p_{3}}:=\frac{\theta}{p_{1}}+\frac{1-\theta}{p_{2}} \quad \text { and } \quad m_{3}:=\theta m_{1}+(1-\theta) m_{2} .
$$

Then, there exists a constant $C$ independent of $T$ such that for any $f \in L^{p_{1}}\left(0, T ; H^{m_{1}}(\Omega)\right) \cap$ $L^{p_{2}}\left(0, T ; H^{m_{2}}(\Omega)\right)$ we have

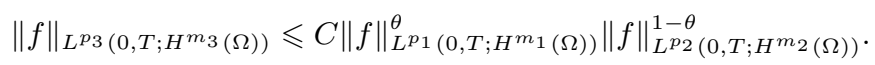

Lemma A.7. Let $s \in[0,1 / 2], \sigma_{0} \in(1 / 2,1], T \in(0,1]$ and $\mathcal{X}_{i}(i=1,2,3)$ be three Banach spaces satisfying $\mathcal{X}_{1} \mathcal{X}_{2} \hookrightarrow \mathcal{X}_{3}$ (i.e., there exists $C>0$ such that for all $g_{1} \in \mathcal{X}_{1}$ and all $g_{2} \in \mathcal{X}_{2}$, we have $\left.\left\|g_{1} g_{2}\right\|_{\mathcal{X}_{3}} \leqslant C\left\|g_{1}\right\|_{\mathcal{X}_{1}}\left\|g_{2}\right\|_{\mathcal{X}_{2}}\right)$. Then, there exists a constant $C$ independent of $T$ such that

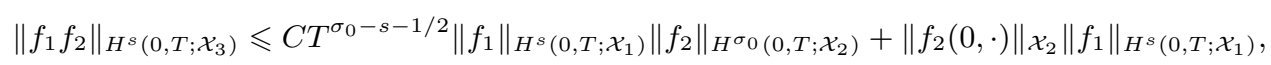

for all $f_{1} \in H^{s}\left(0, T ; \mathcal{X}_{1}\right)$ and all $f_{2} \in H^{\sigma_{0}}\left(0, T ; \mathcal{X}_{2}\right)$.

Proof. We start by observing that

$$
\left\|f_{1} f_{2}\right\|_{H^{s}\left(0, T ; \mathcal{X}_{3}\right)} \leqslant\left\|f_{1}\left(f_{2}-f_{2}(0)\right)\right\|_{H^{s}\left(0, T ; \mathcal{X}_{3}\right)}+\left\|f_{2}(0)\right\|\left\|_{\mathcal{X}_{2}}\right\| f_{1} \|_{H^{s}\left(0, T ; \mathcal{X}_{1}\right)},
$$

so it suffices to prove that

$$
\left\|f_{1}\left(f_{2}-f_{2}(0)\right)\right\|_{H^{s}\left(0, T ; \mathcal{X}_{3}\right)} \leqslant C T^{\sigma_{0}-s-1 / 2}\left\|f_{1}\right\|_{H^{s}\left(0, T ; \mathcal{X}_{1}\right)}\left\|f_{2}\right\|_{H^{\sigma_{0}\left(0, T ; \mathcal{X}_{2}\right)}} .
$$

First, from A.11, we have

$$
\left\|f_{1}\left(f_{2}-f_{2}(0)\right)\right\|_{H^{s}\left(0, T ; \mathcal{X}_{3}\right)} \leqslant T^{-s+1 / 2}\left\|\left(f_{1}\right)^{*}\left(\left(f_{2}\right)^{*}-\left(f_{2}\right)^{*}(0)\right)\right\|_{H^{s}\left(0,1 ; \mathcal{X}_{3}\right)} .
$$


Then, we use A.12 (for $T=1$ and $s=\sigma_{0}$ ):

$$
\begin{aligned}
\left\|\left(f_{1}\right)^{*}\left(\left(f_{2}\right)^{*}-\left(f_{2}\right)^{*}(0)\right)\right\|_{H^{s}\left(0,1 ; \mathcal{X}_{3}\right)} & \leqslant C\left\|\left(f_{1}\right)^{*}\right\|_{H^{s}\left(0,1 ; \mathcal{X}_{1}\right)}\left\|\left(f_{2}\right)^{*}-\left(f_{2}\right)^{*}(0)\right\|_{H^{\sigma_{0}\left(0,1 ; \mathcal{X}_{2}\right)}} \\
& \leqslant C\left\|\left(f_{1}\right)^{*}\right\|_{H^{s}\left(0,1 ; \mathcal{X}_{1}\right)}\left\lfloor\left(f_{2}\right)^{*}-\left(f_{2}\right)^{*}(0)\right\rfloor_{\sigma_{0}, 2,(0,1), \mathcal{X}_{2}} \\
& \left.\leqslant C T^{-1 / 2}\left\|f_{1}\right\|_{H^{s}\left(0, T ; \mathcal{X}_{1}\right)} T^{\sigma_{0}-1 / 2}\left\lfloor f_{2}-f_{2}(0)\right\rfloor\right\rfloor_{\sigma_{0}, 2,(0, T), \mathcal{X}_{2}} \\
& \leqslant C T^{-1 / 2}\left\|f_{1}\right\|_{H^{s}\left(0, T ; \mathcal{X}_{1}\right)} T^{\sigma_{0}-1 / 2}\left\lfloor f_{2}\right\rfloor_{\sigma_{0}, 2,(0, T), \mathcal{X}_{2}} .
\end{aligned}
$$

Then, estimate A.19 follows from A.20, and this last inequality .

Lemma A.8. There exists $C>0$ such that

$$
\|f\|_{H^{3 / 4}\left(0, T ; L^{2}(\partial \Omega)\right)}+\|\nabla f\|_{H^{1 / 4}\left(0, T ; L^{2}(\partial \Omega)\right)} \leqslant C\left(\|f\|_{L^{2}\left(0, T ; H^{2}(\Omega)\right)}+\|f\|_{H^{1}\left(0, T ; L^{2}(\Omega)\right)}\right),
$$

for all $f \in L^{2}\left(0, T ; H^{2}(\Omega)\right) \cap H^{1}\left(0, T ; L^{2}(\Omega)\right)$ and all $T \in(0,1)$.

Proof. This result has been proved in 23] (p.9, Theorem 2.1 with $r=2$ and $s=1$ ). In order to prove that $C$ can be taken independent of $T$ if $T<1$, we need to prove the same result differently.

Let us first prove this result for $T=1$. We use a continuous extension operator

$$
P: L^{2}\left(0,1 ; H^{2}(\Omega)\right) \cap H^{1}\left(0,1 ; L^{2}(\Omega)\right) \rightarrow L^{2}\left(\mathbb{R} ; H^{2}(\Omega)\right) \cap H^{1}\left(\mathbb{R} ; L^{2}(\Omega)\right) .
$$

We write in the proof

$$
F:=P(f)
$$

Then, there exists $C>0$ such that

$$
\|F\|_{L^{2}\left(\mathbb{R} ; H^{2}(\Omega)\right.} \leqslant C\|f\|_{L^{2}\left(0,1 ; H^{2}(\Omega)\right)} \quad \text { and } \quad\|F\|_{H^{1}\left(\mathbb{R} ; L^{2}(\Omega)\right)} \leqslant C\|f\|_{H^{1}\left(0,1 ; L^{2}(\Omega)\right)} .
$$

Let us set

$$
\widehat{F}(\tau, x):=\int_{\mathbb{R}} e^{-i \tau t} F(t, x) d t, \forall(\tau, x) \in \mathbb{R} \times \Omega .
$$

Then, if $\rho \in C^{2}(\bar{\Omega})$ such that $\partial_{n} \rho=1$ on $\partial \Omega$, we deduce

$$
\begin{aligned}
& \|\nabla F\|_{H^{1 / 4}\left(0,1 ; L^{2}(\partial \Omega)\right)}^{2} \leqslant C\|\nabla F\|_{H^{1 / 4}\left(\mathbb{R} ; L^{2}(\partial \Omega)\right)}^{2}=C \int_{\mathbb{R}} \int_{\partial \Omega}(1+|\tau|)^{1 / 2}|\nabla \widehat{F}|^{2}(\tau, x) \partial_{n} \rho(x) d x d \tau \\
& =C \int_{\mathbb{R}} \int_{\Omega}(1+|\tau|)^{1 / 2} 2 \Re\left[\nabla^{2} \widehat{F} \overline{\nabla \widehat{F}} \cdot \nabla \rho\right](\tau, x) d x d \tau+C \int_{\mathbb{R}} \int_{\Omega} \Delta \rho(x)(1+|\tau|)^{1 / 2}|\nabla \widehat{F}(\tau, x)|^{2} d x d \tau \\
& \leqslant C\left(\|\widehat{F}\|_{L^{2}\left(\mathbb{R} ; H^{2}(\Omega)\right)}\|\widehat{F}\|_{H^{1 / 2}\left(\mathbb{R} ; H^{1}(\Omega)\right)}+\|\widehat{F}\|_{H^{1 / 2}\left(\mathbb{R} ; H^{1}(\Omega)\right)}^{2}\right) .
\end{aligned}
$$

From this, we deduce

$$
\begin{aligned}
\|\nabla F\|_{H^{1 / 4}\left(0,1 ; L^{2}(\partial \Omega)\right)}^{2} & \leqslant C\left(\|\widehat{F}\|_{L^{2}\left(\mathbb{R} ; H^{2}(\Omega)\right)}^{3 / 2}\|\widehat{F}\|_{H^{1}\left(\mathbb{R} ; L^{2}(\Omega)\right)}^{1 / 2}+\|\widehat{F}\|_{L^{2}\left(\mathbb{R} ; H^{2}(\Omega)\right)}\|\widehat{F}\|_{H^{1}\left(\mathbb{R} ; L^{2}(\Omega)\right)}\right) \\
& \leqslant C\left(\|f\|_{L^{2}\left(0,1 ; H^{2}(\Omega)\right)}^{3 / 2}\|f\|_{H^{1}\left(0,1 ; L^{2}(\Omega)\right)}^{1 / 2}+\|f\|_{L^{2}\left(0,1 ; H^{2}(\Omega)\right)}\|f\|_{H^{1}\left(0,1 ; L^{2}(\Omega)\right)}\right)
\end{aligned}
$$

which, in particular, implies A.21.

Let us now take any $T \in(0,1)$. This proof follows the steps of the proof of Lemma A.5. First we observe that

$$
\|\nabla f\|_{L^{2}\left(0, T ; L^{2}(\partial \Omega)\right)} \leqslant C\|f\|_{L^{2}\left(0, T ; H^{2}(\Omega)\right)} .
$$

where $C$ is independent of $T$. Then, from A.9

$$
\begin{aligned}
&\lfloor\nabla f\rfloor_{1 / 4,2,(0, T), L^{2}(\partial \Omega)}^{2}=T^{1 / 2}\left\lfloor\nabla f^{*}\right\rfloor_{1 / 4,2,(0,1), L^{2}(\partial \Omega)}^{2}=T^{1 / 2}\left\lfloor\nabla f^{*}-\oint \nabla f^{*}\right\rfloor_{1 / 4,2,(0,1), L^{2}(\partial \Omega)}^{2} \\
& \leqslant T^{1 / 2}\left\|\nabla f^{*}-\oint \nabla f^{*}\right\|_{H^{1 / 4}\left(0,1 ; L^{2}(\partial \Omega)\right)}^{2} .
\end{aligned}
$$


We use here $\mathrm{A} .22$ applied to the function $\nabla f^{*}-\oint \nabla f^{*}$ and the Wirtinger-Poincaré inequality:

$$
\begin{aligned}
& \lfloor\nabla f\rfloor_{1 / 4,2,(0, T), L^{2}(\partial \Omega)}^{2} \\
\leqslant & C T^{1 / 2}\left(\left\|f^{*}-\oint f^{*}\right\|_{L^{2}\left(0,1 ; H^{2}(\Omega)\right)}^{3 / 2}\left\|f^{*}-\oint f^{*}\right\|_{H^{1}\left(0,1 ; L^{2}(\Omega)\right)}^{1 / 2}+\left\|f^{*}-\oint f^{*}\right\|_{L^{2}\left(0,1 ; H^{2}(\Omega)\right)}\left\|f^{*}-\oint f^{*}\right\|_{H^{1}\left(0,1 ; L^{2}(\Omega)\right)}\right) \\
\leqslant & C T^{1 / 2}\left(\left\|f^{*}-\oint f^{*}\right\|_{L^{2}\left(0,1 ; H^{2}(\Omega)\right)}^{3 / 2}\left\|\partial_{t^{*}} f^{*}\right\|_{L^{2}\left(0,1 ; L^{2}(\Omega)\right)}^{1 / 2}+\left\|f^{*}-\oint f^{*}\right\|_{L^{2}\left(0,1 ; H^{2}(\Omega)\right)}\left\|\partial_{t^{*}} f^{*}\right\|_{L^{2}\left(0,1 ; L^{2}(\Omega)\right)}\right)
\end{aligned}
$$

Using A.6 and A.7 and the above estimate, we deduce

$$
\begin{aligned}
\lfloor\nabla f\rfloor_{1 / 4,2,(0, T), L^{2}(\partial \Omega)}^{2} \leqslant C T^{1 / 2}\left(T^{-3 / 4} \| f-\oint\right. & f\left\|_{L^{2}\left(0, T ; H^{2}(\Omega)\right)}^{3 / 2} T^{1 / 4}\right\| \partial_{t} f \|_{L^{2}\left(0, T ; L^{2}(\Omega)\right)}^{1 / 2} \\
& \left.+T^{-1 / 2}\|f-\oint f\|_{L^{2}\left(0, T ; H^{2}(\Omega)\right)} T^{1 / 2}\left\|\partial_{t} f\right\|_{L^{2}\left(0, T ; L^{2}(\Omega)\right)}\right) .
\end{aligned}
$$

Combining this estimate with

$$
\|f-\oint f\|_{L^{2}\left(0, T ; H^{2}(\Omega)\right)} \leqslant\|f\|_{L^{2}\left(0, T ; H^{2}(\Omega)\right)} .
$$

we deduce that

$$
\lfloor\nabla f\rfloor_{1 / 4,2,(0, T), L^{2}(\partial \Omega)}^{2} \leqslant C\left(\|f\|_{L^{2}\left(0, T ; H^{2}(\Omega)\right)}^{3 / 2}\|f\|_{H^{1}\left(0, T ; L^{2}(\Omega)\right)}^{1 / 2}+\|f\|_{L^{2}\left(0, T ; H^{2}(\Omega)\right)}\|f\|_{H^{1}\left(0, T ; L^{2}(\Omega)\right)}\right) .
$$

For the last inequality, we have used the fact that The estimate on $\|f\|_{H^{3 / 4}\left(0, T ; L^{2}(\partial \Omega)\right)}$ can be obtained in a similar way.

Lemma A.9. For any $f \in H^{1 / 3}\left(0, T ; L^{2}(\Omega)\right) \cap L^{2}\left(0, T ; H^{1}(\Omega)\right)$,

$$
\|f\|_{H^{1 / 8}\left(0, T ; L^{2}(\partial \Omega)\right)} \leqslant\|f\|_{L^{2}\left(0, T ; L^{2}(\partial \Omega)\right)}+C T^{1 / 24}\|f\|_{H^{1 / 3}\left(0, T ; L^{2}(\Omega)\right)}^{1 / 2}\|f\|_{L^{2}\left(0, T ; H^{1}(\Omega)\right)}^{1 / 2} .
$$

Proof. Let $\rho \in C^{2}(\bar{\Omega})$ such that $\partial_{n} \rho=1$ on $\partial \Omega$. Then,

$$
\begin{aligned}
\lfloor f\rfloor_{1 / 8,2,(0, T), L^{2}(\partial \Omega)}^{2}= & \iint_{(0, T)^{2}} \int_{\partial \Omega} \frac{\left|f\left(t_{1}, x\right)-f\left(t_{2}, x\right)\right|^{2}}{\left|t_{1}-t_{2}\right|^{5 / 4}} \partial_{n} \rho d x d t_{1} d t_{2} \\
= & \iint_{(0, T)^{2}} \int_{\Omega} \frac{\nabla \rho(x) \cdot \nabla\left|f\left(t_{1}, x\right)-f\left(t_{2}, x\right)\right|^{2}}{\left|t_{1}-t_{2}\right|^{5 / 4}} d x d t_{1} d t_{2} \\
& +\iint_{(0, T)^{2}} \int_{\Omega} \frac{\Delta \rho(x)\left|f\left(t_{1}, x\right)-f\left(t_{2}, x\right)\right|^{2}}{\left|t_{1}-t_{2}\right|^{5 / 4}} d x d t_{1} d t_{2} \\
& \leqslant C \iint_{(0, T)^{2}} \frac{|| f\left(t_{1}, \cdot\right)-f\left(t_{2}, \cdot\right)\left\|_{L^{2}(\Omega)}\right\| f\left(t_{1}, \cdot\right)-f\left(t_{2}, \cdot\right) \|_{H^{1}(\Omega)}}{\left|t_{1}-t_{2}\right|^{5 / 4}} d t_{1} d t_{2}
\end{aligned}
$$

We apply Cauchy-Schwarz's inequality so the last integral is bounded by :

$$
\begin{aligned}
& C\left(\iint_{(0, T)^{2}} \frac{\left\|f\left(t_{1}, \cdot\right)-f\left(t_{2}, \cdot\right)\right\|_{L^{2}(\Omega)}^{2}}{\left|t_{1}-t_{2}\right|^{5 / 3}} d t_{1} d t_{2}\right)^{1 / 2}\left(\iint_{(0, T)^{2}} \frac{\left\|f\left(t_{1}, \cdot\right)-f\left(t_{2}, \cdot\right)\right\|_{H^{1}(\Omega)}^{2}}{\left|t_{1}-t_{2}\right|^{5 / 6} d t_{2}}\right)^{1 / 2} \\
& \leqslant C\lfloor f\rfloor_{1 / 3,2,(0, T), L^{2}(\Omega)}\left(\iint_{(0, T)^{2}} \frac{\left\|f\left(t_{1}, \cdot\right)\right\|_{H^{1}(\Omega)}^{2}+\left\|f\left(t_{2}, \cdot\right)\right\|_{H^{1}(\Omega)}^{2}}{\left|t_{1}-t_{2}\right|^{5 / 6}} d t_{1} d t_{2}\right)^{1 / 2} \\
& \leqslant C T^{1 / 12}\lfloor f\rfloor_{1 / 3,2,(0, T), L^{2}(\Omega)}\|f\|_{L^{2}\left(0, T ; H^{1}(\Omega)\right)} \leqslant C T^{1 / 12}\|f\|_{H^{1 / 3}\left(0, T ; L^{2}(\Omega)\right)}\|f\|_{L^{2}\left(0, T ; H^{1}(\Omega)\right)}
\end{aligned}
$$

The proof of Lemma A.9 is finished. 


\section{B Construction of smooth data}

The goal of this part is to prove that any data which satisfies $1.23-1.25$ can be approximated by smoother data which satisfies 2.1]-2.4. This construction is used in subsection 3.6 to conclude the proof of Theorem 1.4

Let us consider $\left(V_{0}, \Xi_{1}, G\right)$ which satisfies 1.23 - 1.25 . We will construct a sequence $\left(\left(V_{0}^{k}, \Xi_{1}^{k}, G^{k}\right)\right)_{k}$ which satisfies (2.1)-(2.4) and such that

$$
\begin{gathered}
V_{0}^{k} \rightarrow V_{0} \quad \text { in } H^{2}\left(\Omega_{F}\right), \\
\Xi_{1}^{k} \rightarrow \Xi_{1} \quad \text { in } H^{1+1 / 8}\left(\Omega_{S}\right), \\
G^{k} \rightarrow G \quad \text { in } \quad H^{1}\left(0, T ; L^{2}\left(\Omega_{F}\right)\right) \cap L^{2}\left(0, T ; H^{1 / 2+1 / 8}\left(\Omega_{F}\right)\right) .
\end{gathered}
$$

The proof is divided in four steps.

Step 1: First, we construct $\left(V_{0}^{k}\right)_{k}$ which is given by the following lemma:

Lemma B.1. There exists a sequence $\left(V_{0}^{k}\right)_{k}$ in $H^{4}\left(\Omega_{F}\right)$ satisfying (B.1) and

$$
\begin{gathered}
\operatorname{div} V_{0}^{k}=0 \quad \text { in } \Omega_{F}, \\
V_{0}^{k}=0 \quad \text { on } \partial \Omega, \quad\left(\left(\varepsilon\left(V_{0}^{k}\right) n\right) \times n\right) \times n=0 \quad \text { on } \partial \Omega_{S}, \\
\int_{\partial \Omega_{S}}\left(v_{0} \cdot \nabla\right) V_{0}^{k} \cdot n d \gamma=0 .
\end{gathered}
$$

Proof. Let us define $f_{0}:=-\Delta V_{0}+\nabla Q_{0} \in L^{2}\left(\Omega_{F}\right)$. There exists a sequence $\left(f_{0}^{k}\right)_{k}$ in $H^{2}\left(\Omega_{F}\right)$ which converges to $f_{0}$ in $L^{2}\left(\Omega_{F}\right)$. Next, we introduce $\left(\widetilde{V}_{0}^{k}, \widetilde{Q}_{0}^{k}\right)$ in $H^{4}\left(\Omega_{F}\right) \times H^{3}\left(\Omega_{F}\right)$ solution of

$$
\begin{cases}-\operatorname{div} \mathbb{T}\left(\widetilde{V}_{0}^{k}, \widetilde{Q}_{0}^{k}\right)=f_{0}^{k} & \text { in } \Omega_{F}, \\ \operatorname{div} \widetilde{V}_{0}^{k}=0 & \text { in } \Omega_{F}, \\ \widetilde{V}_{0}^{k}=0 & \text { on } \partial \Omega, \\ \mathbb{T}\left(\widetilde{V}_{0}^{k}, \widetilde{Q}_{0}^{k}\right) n=0 & \text { on } \partial \Omega_{S} .\end{cases}
$$

Since $\left(V_{0}, Q_{0}\right)$ satisfies the same problem with $f_{0}$ instead of $f_{0}^{k}$ in the right hand-side of the first equation, standard elliptic results on the above system yield that the sequence $\left(\widetilde{V}_{0}^{k}\right)_{k}$ converges to $V_{0}$ in $H^{2}\left(\Omega_{F}\right)$. Note that $\widetilde{V}_{0}^{k}$ satisfies B.5.

If, for all $k, \widetilde{V}_{0}^{k}$ satisfies B.6, then we take $V_{0}^{k}=\widetilde{V}_{0}^{k}$ and the proof of the lemma is finished. Otherwise, there exists $k_{0} \in \mathbb{N}$ such that

$$
\int_{\partial \Omega_{S}}\left(v_{0} \cdot \nabla\right) \widetilde{V}_{0}^{k_{0}} \cdot n d \gamma \neq 0 .
$$

In this case, we define

$$
V_{0}^{k}=\widetilde{V}_{0}^{k}-\alpha_{k} \widetilde{V}_{0}^{k_{0}}
$$

where $\alpha_{k} \in \mathbb{R}$ is such that B.6 holds, i.e. $\alpha_{k}$ is given by

$$
\alpha_{k}=\frac{\int_{\partial \Omega_{S}}\left(v_{0} \cdot \nabla\right) \widetilde{V}_{0}^{k} \cdot n d \gamma}{\int_{\partial \Omega_{S}}\left(v_{0} \cdot \nabla\right) \widetilde{V}_{0}^{k_{0}} \cdot n d \gamma} .
$$

Since $\widetilde{V}_{0}^{k} \rightarrow V_{0}$ in $H^{2}\left(\Omega_{F}\right)$ and since $V_{0}$ satisfies the second condition of $1.26, \alpha_{k} \rightarrow 0$. By this way, the sequence $\left(V_{0}^{k}\right)_{k}$ satisfies all the desired properties.

Step 2: Second, we take a sequence $\left(\Xi_{1}^{k}\right)_{k}$ in $H^{3 / 2+1 / 8}\left(\Omega_{S}\right)$ such that

$$
\Xi_{1}^{k}=V_{0}^{k} \text { on } \partial \Omega_{S},
$$


and B.2 holds. This is possible since $2>3 / 2+1 / 8$. We have in particular

$$
\int_{\partial \Omega_{S}} \Xi_{1}^{k} \cdot n d \gamma=0 .
$$

Step 3: Then, we define $Q_{0}^{k}$ as the solution of

$$
\begin{cases}\Delta Q_{0}^{k}=-\nabla V_{0}^{k}:\left(\nabla v_{0}\right)^{*} & \text { in } \Omega_{F}, \\ Q_{0}^{k}=2 \varepsilon\left(V_{0}^{k}\right) n \cdot n & \text { on } \partial \Omega_{S}, \\ \frac{\partial Q_{0}^{k}}{\partial n}=\Delta V_{0}^{k} \cdot n & \text { on } \partial \Omega .\end{cases}
$$

We first notice that $Q_{0}^{k}$ belongs to $H^{3}\left(\Omega_{F}\right)$. Moreover, $Q_{0}$ satisfies

$$
\begin{cases}\Delta Q_{0}=-\nabla V_{0}:\left(\nabla v_{0}\right)^{*} & \text { in } \Omega_{F}, \\ Q_{0}=2 \varepsilon\left(Q_{0}\right) n \cdot n & \text { on } \partial \Omega_{S}, \\ \frac{\partial Q_{0}}{\partial n}=\Delta V_{0} \cdot n & \text { on } \partial \Omega,\end{cases}
$$

and, since $\Delta V_{0}^{k} \rightarrow \Delta V_{0}$ in $L^{2}\left(\Omega_{F}\right)$ and $\operatorname{div} \Delta V_{0}^{k}=\operatorname{div} \Delta V_{0}=0$ in $\Omega_{F}$,

$$
\Delta V_{0}^{k} \cdot n \rightarrow \Delta V_{0} \cdot n \text { in } H^{-1 / 2}\left(\partial \Omega_{F}\right)
$$

Moreover

$$
\nabla V_{0}^{k}:\left(\nabla v_{0}\right)^{*} \rightarrow \nabla V_{0}:\left(\nabla v_{0}\right)^{*} \quad \text { in } L^{2}\left(\Omega_{F}\right), \quad \varepsilon\left(V_{0}^{k}\right) n \cdot n \rightarrow \varepsilon\left(V_{0}\right) n \cdot n \quad \text { in } H^{1 / 2}\left(\partial \Omega_{S}\right) .
$$

Thus, the sequence $\left(Q_{0}^{k}\right)_{k}$ converges to $Q_{0}$ in $H^{1}\left(\Omega_{F}\right)$.

Step 4: Now, we construct a sequence $\left(G^{k}\right)_{k}$ which satisfies B.3 and such that

$$
\begin{gathered}
\operatorname{div} G^{k}=0 \quad \text { in }(0, T) \times \Omega_{F}, \\
G^{k}(0, \cdot)=-\Delta V_{0}^{k}+\nabla Q_{0}^{k} \quad \text { on } \partial \Omega_{F} .
\end{gathered}
$$

To do so, we use 1.8 to obtain a sequence $\left(H^{k}\right)_{k}$ such that

$$
\begin{gathered}
H^{k} \rightarrow G \text { in } \quad H^{1}\left(0, T ; L^{2}\left(\Omega_{F}\right)\right) \cap L^{2}\left(0, T ; H^{1 / 2+1 / 8}\left(\Omega_{F}\right)\right), \\
\operatorname{div} H^{k}=0 \quad \text { in }(0, T) \times \Omega_{F}, \\
H^{k}(0, \cdot)=0 \quad \text { on } \partial \Omega_{F} .
\end{gathered}
$$

Next, we state the following result

Lemma B.2. We consider a sequence $\left(u_{k}\right)_{k}$ in $H^{2}\left(\Omega_{F}\right)$ such that $\int_{\partial \Omega_{F}} u_{k} \cdot n d \gamma=0$ and $u_{k} \cdot n \rightarrow 0$ in $H^{-1 / 2}\left(\partial \Omega_{F}\right)$. Then, there exists a sequence $\left(v_{k}\right)_{k}$ in $H^{2}\left(\Omega_{F}\right)$ such that

$$
\begin{gathered}
\operatorname{div} v_{k}=0 \quad \text { in } \Omega_{F}, \\
v_{k}=u_{k} \quad \text { on } \partial \Omega_{F}, \\
v_{k} \rightarrow 0 \quad \text { in } H^{1 / 4+1 / 16}\left(\Omega_{F}\right) .
\end{gathered}
$$

Proof. Let us first construct a sequence $\left(w_{k}\right)_{k}$ in $H^{2}\left(\Omega_{F}\right)$ such that

$$
w_{k}=u_{k} \quad \text { on } \partial \Omega_{F}, \quad w_{k} \rightarrow 0 \quad \text { in } H^{1 / 4+1 / 16}\left(\Omega_{F}\right) .
$$

To do so, we will straighten the boundary of $\partial \Omega_{F}$ by local diffeomorphisms and use cutoff functions on the straightened boundary.

We denote by $B(x, \eta)$ a ball of center $x$ and radius $\eta>0$. Since $\Omega_{F}$ is of class $C^{2}$ and compact, we can straighten locally the boundary by a finite number of $C^{2}$-diffeomorphisms: there exists $N \in \mathbb{N}$ and, for $1 \leqslant i \leqslant N, x_{i} \in \partial \Omega_{F}, \epsilon_{i}>0$ and a $C^{2}$ diffeomorphism $\Psi_{i}$ from $B(0,1)$ on $B\left(x_{i}, \epsilon_{i}\right)$ such that

$$
\partial \Omega_{F} \subset \cup_{1 \leqslant i \leqslant N} B\left(x_{i}, \epsilon_{i}\right)
$$


and

$$
\Psi_{i}\left(B(0,1) \cap \mathbb{R}_{+}^{3}\right)=B\left(x_{i}, \epsilon_{i}\right) \cap \Omega_{F}, \quad \Psi_{i}\left(B(0,1) \cap\left(\mathbb{R}^{2} \times\{0\}\right)\right)=B\left(x_{i}, \epsilon_{i}\right) \cap \partial \Omega_{F} .
$$

We then introduce a partition of unity $\left\{\chi_{i}\right\}_{1 \leqslant i \leqslant N}$ subordinated to $\left\{B\left(x_{i}, \epsilon_{i}\right)\right\}_{1 \leqslant i \leqslant N}$.

Next, we consider a $C^{\infty}$ decreasing function $\theta$ defined in $\mathbb{R}^{+}$such that $\theta(0)=1$ and $\operatorname{supp}(\theta) \subset[0,1]$ and, for all $k \in \mathbb{N}$, we define

$$
\varphi_{k}(x)=\theta\left(x_{3} / a_{k}\right), \forall x \in \mathbb{R}_{3}^{+}
$$

where $\left(a_{k}\right)_{k}$ is a positive decreasing sequence which tends to 0 . By this way, $\operatorname{supp}\left(\varphi_{k}\right) \subset \mathbb{R}^{2} \times\left[0, a_{k}\right]$. Then, we set

$$
w_{k}=\sum_{1 \leqslant i \leqslant N} \chi_{i}\left(\varphi_{k} \circ \Psi_{i}^{-1}\right) u_{k}
$$

We notice that $w_{k}=u_{k}$ on $\partial \Omega_{F}$. Moreover

$$
\left\|w_{k}\right\|_{H^{1 / 4+1 / 16}\left(\Omega_{F}\right)} \leqslant C\left\|\varphi_{k}\right\|_{H^{1 / 4+1 / 16}\left(\left(\mathbb{R}^{2} \times\left[0, a_{k}\right]\right) \cap B(0,1)\right)}\left\|u_{k}\right\|_{H^{2}\left(\Omega_{F}\right)}
$$

and

$$
\left\|\varphi_{k}\right\|_{H^{1 / 4+1 / 16}\left(\left(\mathbb{R}^{2} \times\left[0, a_{k}\right]\right) \cap B(0,1)\right)} \leqslant C\left\|\theta\left(\cdot / a_{k}\right)\right\|_{H^{1 / 4+1 / 16}\left(\left[0, a_{k}\right]\right)} \leqslant C a_{k}^{1 / 4-1 / 16}\|\theta\|_{H^{1 / 4+1 / 16}([0,1])}
$$

according to A.9 applied with $s=1 / 4+1 / 16$. By this way,

$$
\left\|w_{k}\right\|_{H^{1 / 4+1 / 16}\left(\Omega_{F}\right)} \leqslant C a_{k}^{1 / 4-1 / 16}\left\|u_{k}\right\|_{H^{2}\left(\Omega_{F}\right)} .
$$

Thus, choosing the sequence $\left(a_{k}\right)_{k}$ small enough so that $\left(a_{k}^{1 / 4-1 / 16}\left\|u_{k}\right\|_{H^{2}\left(\Omega_{F}\right)}\right)_{k}$ tends to 0 , we get B.11. To end the proof of Lemma B.2. since $\int_{\partial \Omega_{F}} w_{k} \cdot n d \gamma=\int_{\partial \Omega_{F}} u_{k} \cdot n d \gamma=0$, we can define $\left(l_{k}, \pi_{k}\right)$ solution of

$$
\begin{cases}-\Delta l_{k}+\nabla \pi_{k}=0 & \text { in } \Omega_{F}, \\ \operatorname{div} l_{k}=\operatorname{div} w_{k} & \text { in } \Omega_{F}, \\ l_{k}=0 & \text { on } \partial \Omega_{F} .\end{cases}
$$

We have that $l_{k} \rightarrow 0$ in $H^{1 / 4+1 / 16}\left(\Omega_{F}\right)$ and so, if we define $v_{k}:=w_{k}-l_{k}$, we get the desired properties for the sequence $\left(v_{k}\right)_{k}$.

We want to apply this lemma to $u_{k}=-\Delta V_{0}^{k}+\nabla Q_{0}^{k}$. We first notice that

$$
\int_{\partial \Omega_{F}} \Delta V_{0}^{k} \cdot n d \gamma=\int_{\Omega_{F}} \operatorname{div} \Delta V_{0}^{k} d x=0
$$

and

$$
\begin{aligned}
\int_{\partial \Omega_{F}} \nabla Q_{0}^{k} \cdot n d \gamma & =\int_{\Omega_{F}} \Delta Q_{0}^{k} d x=-\int_{\Omega_{F}} \nabla V_{0}^{k}:\left(\nabla v_{0}\right)^{*} d x \\
& =-\int_{\Omega_{F}} \operatorname{div}\left(\left(v_{0} \cdot \nabla\right) V_{0}^{k}\right) d x=-\int_{\partial \Omega_{F}}\left(v_{0} \cdot \nabla\right) V_{0}^{k} \cdot n d \gamma=0
\end{aligned}
$$

By this way, $\int_{\partial \Omega_{F}} u_{k} \cdot n d \gamma=0$.

Moreover,

$$
\nabla Q_{0}^{k} \rightarrow \nabla Q_{0} \text { in } L^{2}\left(\Omega_{F}\right)
$$

and

$$
\Delta Q_{0}^{k}=-\nabla V_{0}^{k}:\left(\nabla v_{0}\right)^{*} \rightarrow-\nabla V_{0}:\left(\nabla v_{0}\right)^{*}=\Delta Q_{0} \text { in } L^{2}\left(\Omega_{F}\right) .
$$

Thus $\nabla Q_{0}^{k} \cdot n \rightarrow \nabla Q_{0} \cdot n$ in $H^{-1 / 2}\left(\partial \Omega_{F}\right)$ and according to B.8, we deduce that

$$
u_{k} \cdot n \rightarrow\left(-\Delta V_{0}+\nabla Q_{0}\right) \cdot n \text { in } H^{-1 / 2}\left(\partial \Omega_{F}\right) .
$$


We notice that the compatibility conditions $1.8,21.9)_{1}$ and $[1.9]_{6}$ imply that $\left(-\Delta V_{0}+\nabla Q_{0}\right) \cdot n=0$ on $\partial \Omega_{F}$. Thus, we can apply Lemma B.2 and we deduce the existence of a sequence denoted $\left(\widetilde{G}_{0}^{k}\right)_{k}$ in $H^{2}\left(\Omega_{F}\right)$ such that

$$
\begin{gathered}
\widetilde{G}_{0}^{k} \rightarrow 0 \quad \text { in } H^{1 / 4+1 / 16}\left(\Omega_{F}\right), \\
\operatorname{div} \widetilde{G}_{0}^{k}=0 \quad \text { in } \Omega_{F}, \\
\widetilde{G}_{0}^{k}=-\Delta V_{0}^{k}+\nabla Q_{0}^{k} \quad \text { on } \partial \Omega_{F} .
\end{gathered}
$$

According to [22, p.21, Theorem 3.2], for each $k \in \mathbb{N}$, there exists

$$
\widetilde{G}^{k} \in H^{1}\left(0, T ; L^{2}\left(\Omega_{F}\right)\right) \cap L^{2}\left(0, T ; H^{1 / 2+1 / 8}\left(\Omega_{F}\right)\right)
$$

with $\operatorname{div} \widetilde{G}^{k}=0$ in $(0, T) \times \Omega_{F}$ such that

$$
\widetilde{G}^{k}(0, \cdot)=\widetilde{G}_{0}^{k} \text { in } \Omega_{F} .
$$

Moreover, there exists a constant $C>0$ such that, for all $k \in \mathbb{N}$

$$
\left\|\widetilde{G}^{k}\right\|_{H^{1}\left(0, T ; L^{2}\left(\Omega_{F}\right)\right)}+\left\|\widetilde{G}^{k}\right\|_{L^{2}\left(0, T ; H^{1 / 2+1 / 8}\left(\Omega_{F}\right)\right)} \leqslant C\left\|\widetilde{G}_{0}^{k}\right\|_{H^{1 / 4+1 / 16}\left(\Omega_{F}\right)} .
$$

By this way, $\left(\widetilde{G}^{k}\right)_{k}$ satisfies the following properties

$$
\begin{gathered}
\widetilde{G}^{k} \rightarrow 0 \quad \text { in } H^{1}\left(0, T ; L^{2}\left(\Omega_{F}\right)\right) \cap L^{2}\left(0, T ; H^{1 / 2+1 / 8}\left(\Omega_{F}\right)\right) \\
\operatorname{div} \widetilde{G}^{k}=0 \quad \text { in }(0, T) \times \Omega_{F}, \\
\widetilde{G}^{k}(0, \cdot)=-\Delta V_{0}^{k}+\nabla Q_{0}^{k} \quad \text { on } \partial \Omega_{F} .
\end{gathered}
$$

Now, if we define, for all $k \in \mathbb{N}, G^{k}:=H^{k}+\widetilde{G}^{k}$, we get that $\left(G^{k}\right)_{k}$ satisfies B.3, B.9 and B.10.

Step 5: At last, we define

$$
V_{1}^{k}=\operatorname{div} \mathbb{T}\left(V_{0}^{k}, Q_{0}^{k}\right)+G^{k}(0, \cdot) .
$$

According to B.4, B.7 1 and $\mathrm{B} .9$, we have that $\operatorname{div} V_{1}^{k}=\nabla V_{0}^{k}:\left(\nabla v_{0}\right)^{*}$ in $\Omega_{F}$ and according to B.10, $V_{1}^{k}=0$ on $\partial \Omega_{F}$.

\section{References}

[1] BAdra, M., And TAKahashi, T. Feedback boundary stabilization of 2D fluid-structure interaction systems. Discrete Contin. Dyn. Syst. 37, 5 (2017), 2315-2373.

[2] BeIRÃo DA VEIGA, H. On the existence of strong solutions to a coupled fluid-structure evolution problem. J. Math. Fluid Mech. 6, 1 (2004), 21-52.

[3] Boulakia, M. Existence of weak solutions for an interaction problem between an elastic structure and a compressible viscous fluid. J. Math. Pures Appl. (9) 84, 11 (2005), 1515-1554.

[4] Boulakia, M. Existence of weak solutions for the three-dimensional motion of an elastic structure in an incompressible fluid. J. Math. Fluid Mech. 9, 2 (2007), 262-294.

[5] Boulakia, M., ANd Guerrero, S. Regular solutions of a problem coupling a compressible fluid and an elastic structure. J. Math. Pures Appl. (9) 94, 4 (2010), 341-365.

[6] Boulakia, M., And Guerrero, S. On the interaction problem between a compressible fluid and a Saint-Venant Kirchhoff elastic structure. Adv. Differential Equations 22, 1-2 (2017), 1-48.

[7] Boulakia, M., Schwindt, E. L., And Takahashi, T. Existence of strong solutions for the motion of an elastic structure in an incompressible viscous fluid. Interfaces Free Bound. 14, 3 (2012), 273306.

[8] Chambolle, A., Desjardins, B. т., Esteban, M. J., And Grandmont, C. Existence of weak solutions for the unsteady interaction of a viscous fluid with an elastic plate. J. Math. Fluid Mech. 7, 3 (2005), 368-404.

[9] Coutand, D., And Shkoller, S. Motion of an elastic solid inside an incompressible viscous fluid. Arch. Ration. Mech. Anal. 176, 1 (2005), 25-102. 
[10] Coutand, D., And Shkoller, S. The interaction between quasilinear elastodynamics and the Navier-Stokes equations. Arch. Ration. Mech. Anal. 179, 3 (2006), 303-352.

[11] Desjardins, B., Esteban, M. J., Grandmont, C., And Le Tallec, P. Weak solutions for a fluid-elastic structure interaction model. Rev. Mat. Complut. 14, 2 (2001), 523-538.

[12] Grandmont, C. Existence of weak solutions for the unsteady interaction of a viscous fluid with an elastic plate. SIAM J. Math. Anal. 40, 2 (2008), 716-737.

[13] Grandmont, C., And Hillairet, M. Existence of global strong solutions to a beam-fluid interaction system. Arch. Ration. Mech. Anal. 220, 3 (2016), 1283-1333.

[14] Grandmont, C., Hillairet, M., and Lequeurre, J. Existence of local strong solutions to fluidbeam and fluid-rod interaction systems. to appear in Ann. Inst. H. Poincaré Anal. Non Linéaire.

[15] Grubb, G., And Solonnikov, V. A. Boundary value problems for the nonstationary Navier-Stokes equations treated by pseudo-differential methods. Math. Scand. 69, 2 (1991), 217-290 (1992).

[16] Horgan, C. O. Korn's inequalities and their applications in continuum mechanics. SIAM Rev. 37, 4 (1995), 491-511.

[17] Ignatova, M., Kukavica, I., Lasiecka, I., And Tuffaha, A. Small data global existence for a fluid-structure model. Nonlinearity 30, 2 (2017), 848-898.

[18] Kukavica, I., And Tuffaha, A. Solutions to a fluid-structure interaction free boundary problem. Discrete Contin. Dyn. Syst. 32, 4 (2012), 1355-1389.

[19] Kukavica, I., And Tuffaha, A. Well-posedness for the compressible Navier-Stokes-Lamé system with a free interface. Nonlinearity 25, 11 (2012), 3111-3137.

[20] Kukavica, I., Tuffaha, A., And Ziane, M. Strong solutions to a Navier-Stokes-Lamé system on a domain with a non-flat boundary. Nonlinearity 24, 1 (2011), 159-176.

[21] Lasiecka, I., Lions, J.-L., And Triggiani, R. Nonhomogeneous boundary value problems for second order hyperbolic operators. J. Math. Pures Appl. (9) 65, 2 (1986), 149-192.

[22] Lions, J.-L., AND Magenes, E. Non-homogeneous boundary value problems and applications. Vol. I. Springer-Verlag, New York-Heidelberg, 1972. Translated from the French by P. Kenneth, Die Grundlehren der mathematischen Wissenschaften, Band 181.

[23] Lions, J.-L., AND Magenes, E. Non-homogeneous boundary value problems and applications. Vol. II. Springer-Verlag, New York-Heidelberg, 1972. Translated from the French by P. Kenneth, Die Grundlehren der mathematischen Wissenschaften, Band 182.

[24] Raymond, J.-P. Feedback stabilization of a fluid-structure model. SIAM J. Control Optim. 48, 8 (2010), 5398-5443.

[25] Raymond, J.-P., and Vanninathan, M. A fluid-structure model coupling the Navier-Stokes equations and the Lamé system. J. Math. Pures Appl. (9) 102, 3 (2014), 546-596.

[26] Tucsnak, M., And Weiss, G. Observation and control for operator semigroups. Birkhäuser Advanced Texts: Basler Lehrbücher. [Birkhäuser Advanced Texts: Basel Textbooks]. Birkhäuser Verlag, Basel, 2009. 